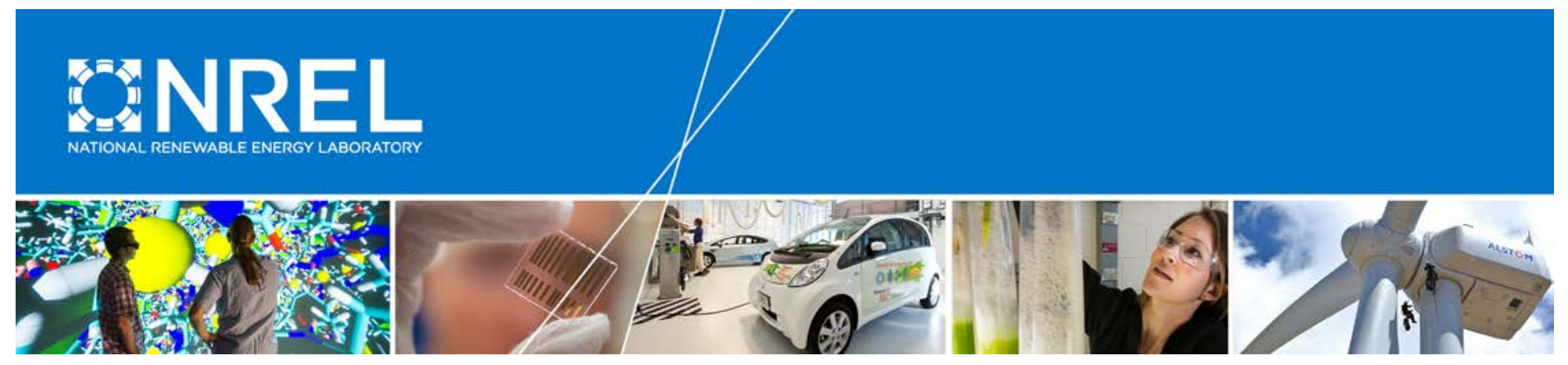

\title{
Characterization of PTO and Idle Behavior for Utility Vehicles
}

Arnaud Konan, Adam Duran, Kenneth Kelly, Eric Miller, and Robert Prohaska National Renewable Energy Laboratory

NREL is a national laboratory of the U.S. Department of Energy Office of Energy Efficiency \& Renewable Energy Operated by the Alliance for Sustainable Energy, LLC

This report is available at no cost from the National Renewable Energy Laboratory (NREL) at www.nrel.gov/publications.

Technical Report

NREL/TP-5400-66747

September 2017 


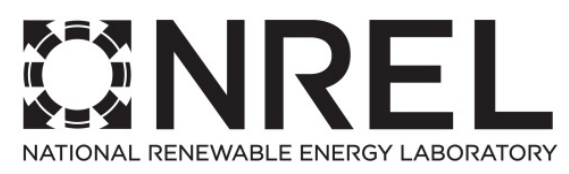

\title{
Characterization of PTO and Idle Behavior for Utility Vehicles
}

\author{
Arnaud Konan, Adam Duran, Kenneth Kelly, \\ Eric Miller, and Robert Prohaska \\ National Renewable Energy Laboratory \\ Prepared under the direction of the U.S. Environmental \\ Protection Agency (EPA) by the National Renewable \\ Energy Laboratory (NREL) as a product of Interagency \\ Agreement IAG-14-1954 and Task No. WFGY.1000
}

NREL is a national laboratory of the U.S. Department of Energy Office of Energy Efficiency \& Renewable Energy Operated by the Alliance for Sustainable Energy, LLC

This report is available at no cost from the National Renewable Energy Laboratory (NREL) at www.nrel.gov/publications.

National Renewable Energy Laboratory 15013 Denver West Parkway Golden, CO 80401 303-275-3000 • www.nrel.gov

\section{Technical Report}

NREL/TP-5400-66747

September 2017

Contract No. DE-AC36-08GO28308 


\section{NOTICE}

This report was prepared as an account of work sponsored by an agency of the United States government. Neither the United States government nor any agency thereof, nor any of their employees, makes any warranty, express or implied, or assumes any legal liability or responsibility for the accuracy, completeness, or usefulness of any information, apparatus, product, or process disclosed, or represents that its use would not infringe privately owned rights. Reference herein to any specific commercial product, process, or service by trade name, trademark, manufacturer, or otherwise does not necessarily constitute or imply its endorsement, recommendation, or favoring by the United States government or any agency thereof. The views and opinions of authors expressed herein do not necessarily state or reflect those of the United States government or any agency thereof.

This report is available at no cost from the National Renewable Energy Laboratory (NREL) at www.nrel.gov/publications.

Available electronically at SciTech Connect http:/www.osti.gov/scitech

Available for a processing fee to U.S. Department of Energy and its contractors, in paper, from:

U.S. Department of Energy

Office of Scientific and Technical Information

P.O. Box 62

Oak Ridge, TN 37831-0062

OSTI http://www.osti.gov

Phone: 865.576.8401

Fax: 865.576.5728

Email: reports@osti.gov

Available for sale to the public, in paper, from:

U.S. Department of Commerce

National Technical Information Service

5301 Shawnee Road

Alexandria, VA 22312

NTIS http://www.ntis.gov

Phone: 800.553 .6847 or 703.605 .6000

Fax: 703.605.6900

Email: orders@ntis.gov 


\section{List of Acronyms}

AM

AT

CAN

dPTO

ePTO

NREL

PG\&E

PTO

RPM
Altec material handler truck

Altec trouble truck

controller area network

diesel engine powered PTO

battery powered PTO

National Renewable Energy Laboratory

Pacific Gas and Electric

power take-off

revolutions per minute 


\section{Table of Contents}

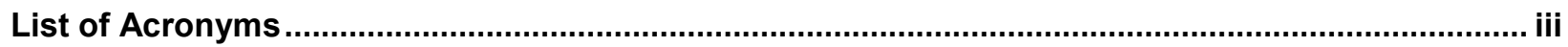

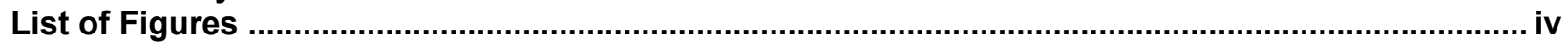

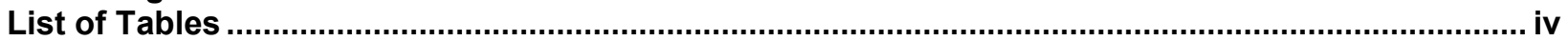

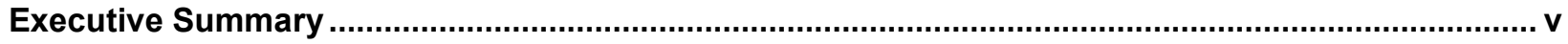

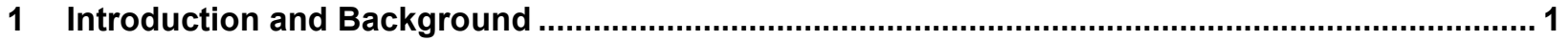

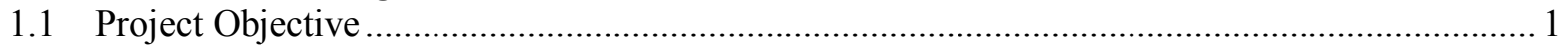

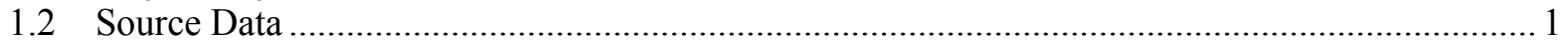

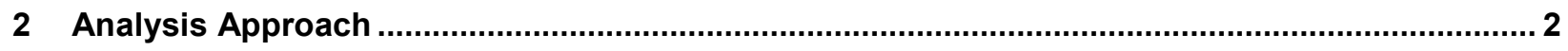

2.1 Engine Speed Method for Characterizing PTO and Idle Operation (RPM Method) ................... 4

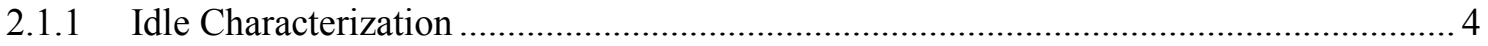

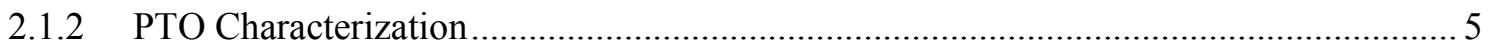

2.2 PTO Channel Method for Characterizing PTO and Idle Operation ....................................... 6

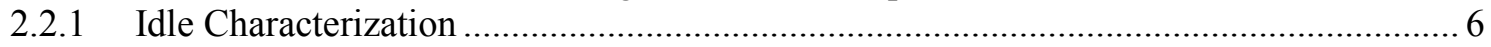

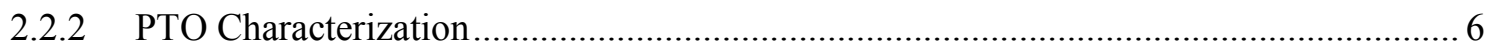

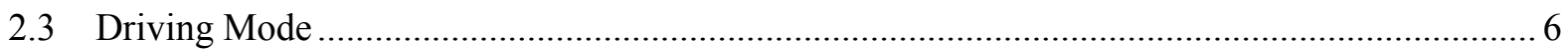

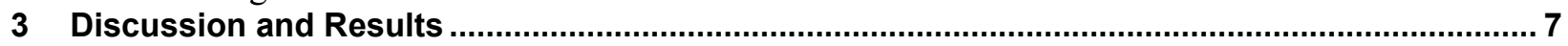

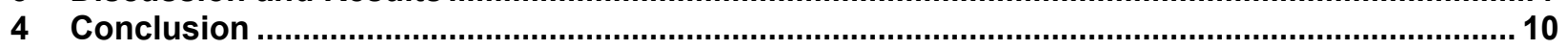

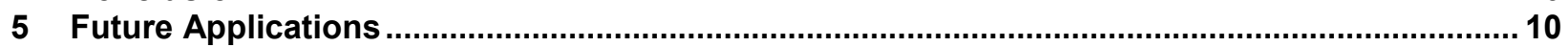

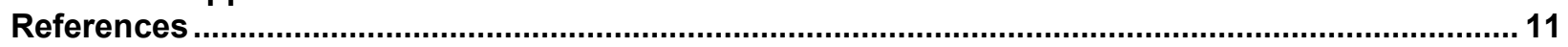

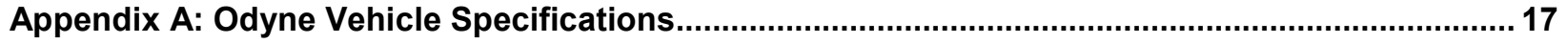

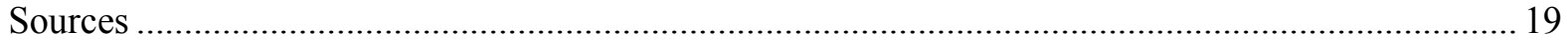

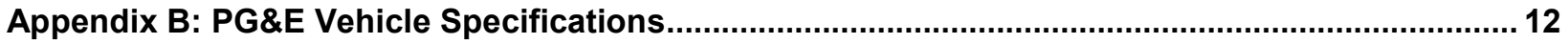

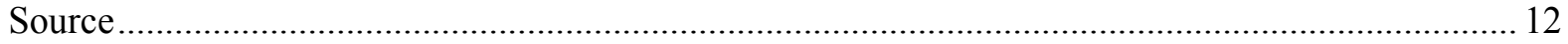

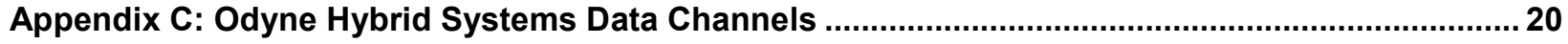

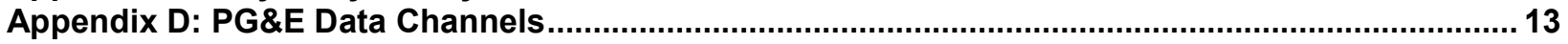

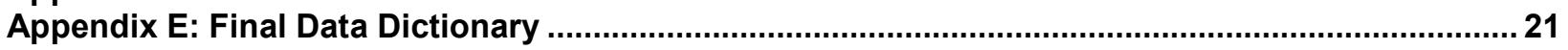

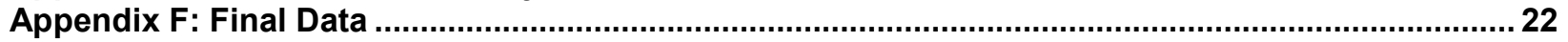

\section{List of Figures}

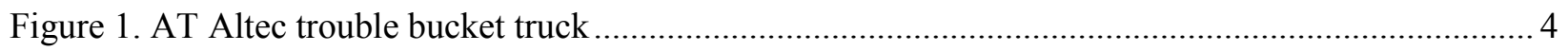

Figure 2. AM Altec material handler double-bucket truck .............................................................. 5

Figure 3. Illustration of the RPM method, histogram of engine RPM where vehicle speed is zero, but

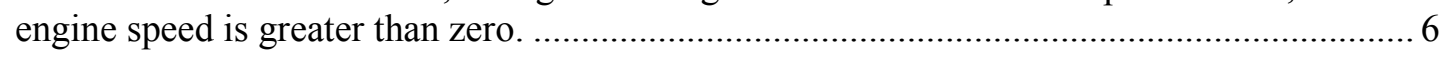

Figure 4. Comparison of PTO and engine RPM derived cumulative idle and PTO operation.................... 7

Figure 5. Percent of time [left] and fuel [right] spent in each operating mode ........................................ 8

Figure 6. Distribution of PTO time for all vehicles in this study (PG\&E and Odyne combined) ............... 9

Figure 7. Distribution of idle time for all vehicles in this study (PG\&E and Odyne combined) ................. 9

\section{List of Tables}

Table 1. Summary of Source Vehicle Data...................................................................................... 2

Table 2. Odyne Mode Determination Conditional Logic ................................................................ 3 


\section{Executive Summary}

This report presents the results of analyses performed on utility vehicle data composed primarily of aerial lift bucket trucks sampled from the National Renewable Energy Laboratory's Fleet DNA database to characterize power takeoff (PTO) and idle operating behavior for utility trucks. Two major data sources were examined in this study: a 75-vehicle sample of Odyne electric PTO (ePTO)-equipped vehicles drawn from multiple fleets spread across the United States and 10 conventional PTO-equipped Pacific Gas and Electric fleet vehicles operating in California. Novel data mining approaches were developed to identify PTO and idle operating states for each of the datasets using telematics and controller area network/onboard diagnostics data channels. These methods were applied to the individual datasets and aggregated to develop utilization curves and distributions describing PTO and idle behavior in both absolute and relative operating terms. This report also includes background information on the source vehicles, development of the analysis methodology, and conclusions regarding the study's findings. 


\section{Introduction and Background}

\subsection{Project Objective}

In an effort to develop a better understanding of power take-off (PTO) and engine idling behavior of U.S. commercial vehicles, the U.S. Environmental Protection Agency through an Interagency Agreement with the U.S. Department of Energy tasked National Renewable Energy Laboratory (NREL) researchers with performing focused analyses characterizing the real-world commercial vehicle operating data stored in NREL's Fleet DNA database. This online database and tool - available at http://www.nrel.gov/fleetdna — provide both static and interactive data summaries and visualizations representative of the real-world "genetics" for medium- and heavyduty commercial fleet vehicles. One-hertz $(1-\mathrm{Hz})$ controller area network (CAN) recorded data for a subset of aerial lift utility vehicles (referred to here as "utility vehicles") configured with conventional hydraulic or hybrid PTO systems in the Fleet DNA database were analyzed to develop a distribution of PTO event durations as well as a distribution describing the proportion of PTO usage as a percent of total vehicle operating time. A similar subset of data was sampled from Fleet DNA to develop corresponding distributions of in-gear and out-of-gear idle behavior.

\subsection{Source Data}

In collaboration with fleet and industry partners across the country, the Fleet Testing and Evaluation team in NREL's Transportation and Hydrogen Systems Center focuses on evaluating the real-world performance of alternative fuel and advanced vehicle technologies deployed in medium- and heavy-duty commercial fleet vehicles throughout the United States. NREL's Fleet Testing and Evaluation team instruments vehicles operating in the field with data recording devices to capture 1-Hz telematics and CAN data. The data are then used to calculate in-use fuel economy and perform drive-cycle characterization and system-level duty-cycle analysis, which can be complemented with chassis dynamometer emissions and fuel economy testing. These results are used to provide feedback to stakeholders such as fleets, technology providers, researchers, and government agencies, helping to inform and provide insight on the performance of advanced technology and fuels operating under real world conditions. The data for these evaluation projects are stored in the Fleet DNA database along with additional externally sourced data that have been supplied by Fleet DNA project partners. For more information about the Fleet DNA database project, please consult [1].

While the Fleet DNA database includes a wide range of vehicle vocations, utility vehicles were identified as the focus of this study. The final statistics in this report are limited to data from 75 Odyne vehicles, which had useful data for this analysis, and 10 utility vehicles from Pacific Gas and Electric (PG\&E), as shown in Table 1. 
Table 1. Summary of Source Vehicle Data

\begin{tabular}{|l|l|l|}
\hline Project & Odyne & PG\&E \\
\hline Vocation & Utility vehicle & Utility vehicle \\
\hline Number of vehicles & 75 & 10 \\
\hline Collection period & 7 months & 4 months \\
\hline Location & $\begin{array}{l}\text { Various U.S. cities/states } \\
{[2]}\end{array}$ & California \\
\hline Number of vehicle days & 1,164 & 406 \\
\hline Total miles & 29,025 & 41,400 \\
\hline Fuel type & Diesel & Diesel \\
\hline Class 5 & 0 & 5 \\
\hline Class 6 & 10 & 0 \\
\hline Class 7 & 33 & 0 \\
\hline Class 8 & 32 & 5 \\
\hline Total & 75 & 10 \\
\hline
\end{tabular}

The utility vehicle data were collected in two separate studies. For the PG\&E fleet, NREL installed data loggers on five class 5 "Altec Trouble Trucks" (AT) bucket trucks and five class 8 "Altec Material Handlers" (AM) double-bucket trucks, collecting 1-Hz data from vehicles operating in the PG\&E service area in California. Appendix A includes vehicle specifications and Appendix B includes a detailed list of data channels. In addition to the PG\&E data, 1-Hz data from Odyne vehicles were provided by the Electric Power Research Institute under a U.S. Department of Energy-funded electric vehicle deployment program awarded to the South Coast Air Quality Management District [2]. The Odyne vehicles analyzed in this study operated in multiple fleets spread across the United States. Appendices C and D include the vehicle specifications and data channels for the Odyne vehicles. Appendices E and F contain the associated data dictionary and results generated from both the PG\&E and Odyne vehicles.

\section{Analysis Approach}

To determine the correct operating range of engine speed corresponding to engine idle events and engine PTO operation for each vehicle, custom data mining techniques were developed for each of the two major datasets. Using exploratory data analysis, engine speed ranges corresponding to idle and PTO operation were identified for each vehicle in the datasets. Once engine speed values were identified for each vehicle, a margin around those values was applied, and the data were reprocessed in a recursive algorithm to identify and aggregate the time and fuel for each operating mode. This involved employing a series of logic statements involving engine speed, brake switch activity, transmission signals, and other supporting information to separate in-gear vs. out-of-gear idle conditions from PTO operation using the engine speed values identified in the data mining exercise. 
For the Odyne hybrid systems dataset, a separate set of logic filters was developed to determine vehicle operating state as a result of nonstandard data parameters being available for analysis. The dataset format resembles SAE J1939, but a few channels custom channels have been added. Notably, global positioning system data are unavailable, and an extra channel was established to describe the general operating state of the truck. This "Mode" signal is not communicated over the vehicle CAN network, but is used by Odyne to aid in data analysis.

The original reported modes are:

- Drive: Vehicle is in drive gear with engine running.

- Park: Vehicle is in park or neutral with the parking brake set.

- Charge: Vehicle is plugged into a charger, and the hybrid battery is being charged.

- Operate: The vehicle is utilizing the PTO functionality of the vehicle or powering other loads such as the heating, ventilating, and air conditioning; lighting; or tool chargers with the electric hybrid PTO (ePTO) system.

Modes were added for field charging and engine-on PTO. The final set of modes relevant to this study is: Drive, Idle (Park), diesel engine-on PTO (dPTO) and electric PTO (ePTO) (batterypowered PTO, engine-off). The logic shown in Table 2 was applied to the Odyne data, which already included basic mode information.

Table 2. Odyne Mode Determination Conditional Logic

\begin{tabular}{|l|l|}
\hline Mode & Conditions \\
\hline Drive & Mode = Drive \\
\hline dPTO & $\begin{array}{l}\text { Mode }=\text { Operating, and Engine Speed }>0 \text { revolutions per minute (RPM), or } \\
\text { Mode }=\text { Park, and Engine Speed }>1,000 \text { RPM }\end{array}$ \\
\hline Idle & Mode = Park, and Engine Speed $>0$ RPM \\
\hline ePTO & Mode = Operating, and Engine Speed $=0$ RPM \\
\hline Park & Mode $=$ Park, and Engine Speed $==0$ RPM \\
\hline
\end{tabular}

Two techniques were evaluated to determine the more robust method for quantifying PTO and idle operation from the available data. These two methods included examining different available data channels using custom logic similar to that developed for identifying vehicle operating state .These methods include:

1. Engine speed method

2. PTO channel method.

These two methods are explained and compared in the following sections. 


\subsection{Engine Speed Method for Characterizing PTO and Idle Operation (RPM Method)}

\subsubsection{Idle Characterization}

For the PG\&E vehicles, idle activity is defined as:

- Vehicle Speed $=0$ miles per hour

○ The vehicle is stationary.

- Engine Speed $>0$ RPM

- The engine is running, and the vehicle is not in a key-off state.

- Engine Speed $<650 \mathrm{RPM}+10 \%$ for AT trucks, and $750 \mathrm{RPM}+10 \%$ for AM trucks

○ The engine is running, but not performing PTO-related tasks.

An Altec trouble truck (AT) is shown in Figure 1, and an Altec material handler (AM) is shown in Figure 2. The differences between vehicle configurations are primarily the number of axles (ATs are a $4 \times 4$ configuration while AMs are $6 \times 6$ configured) and vehicle weight class (ATs are class 5, and AMs are class 8) as well as boom length (35-ft. single boom for ATs, 55-ft. double boom for AMs). The ATs are mostly assigned to "troublemen" who respond to a variety of local grid issues. They spend more time driving and less extended PTO work, but do idle their vehicles a considerable amount of time whereas the AMs are deployed to perform extensive heavy-duty PTO work.

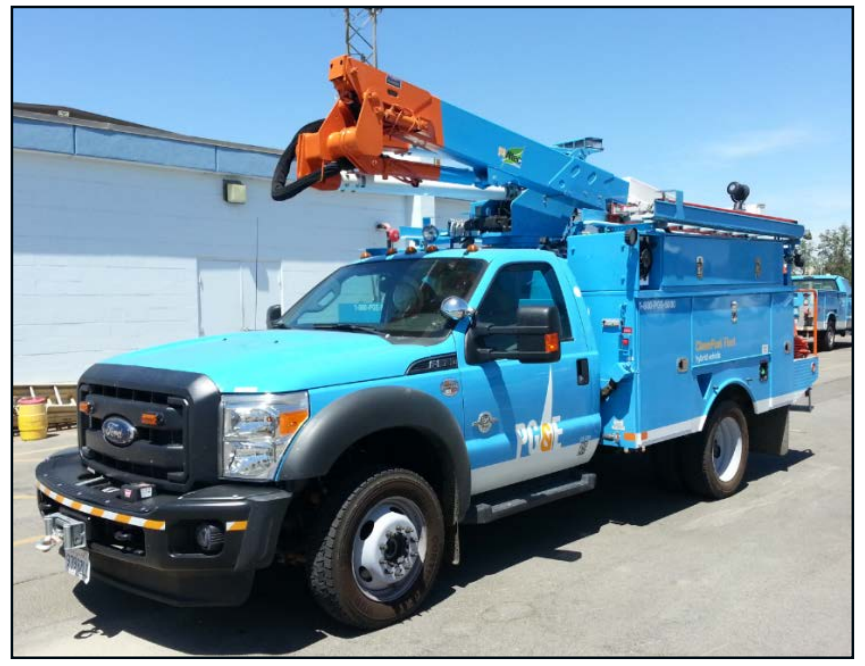

Figure 1. Altec AT trouble bucket truck 


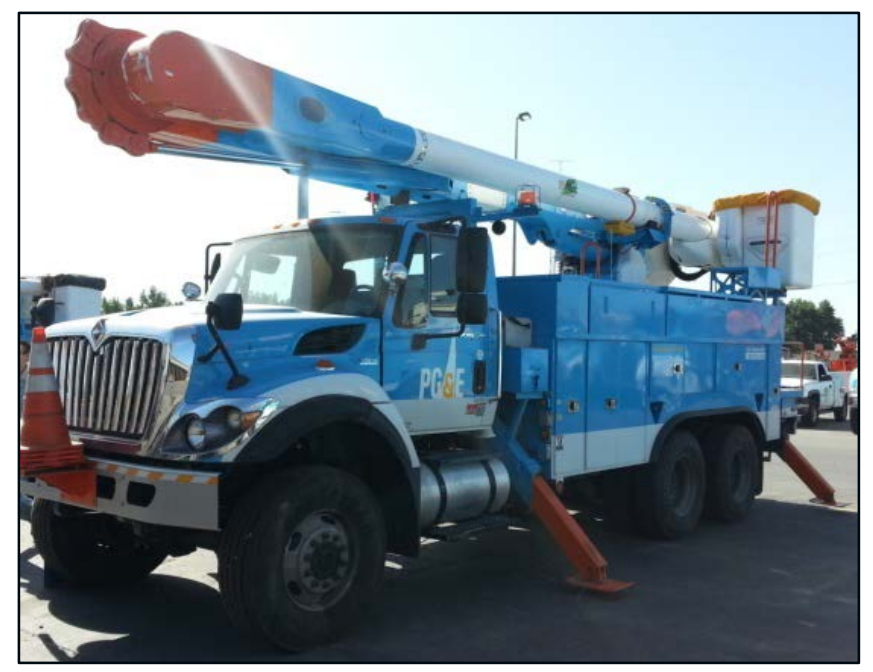

Figure 2. Altec AM material handler double-bucket truck

The logic applied to the PG\&E data ensures that PTO operating time is not included in the idle statistics. However, this logic does not try to differentiate the various types of idling such as ingear idling at traffic lights or parked idling when running the heating, ventilating, and air conditioning system for cabin comfort when at the job site.

For Odyne hybrid vehicles, the "idle" mode was identified as conditions where:

- Vehicle Engine Speed >0 RPM

$\bigcirc$ The vehicle engine must be running.

- $\quad$ Operating Mode $=$ Parked

○ The vehicle is in parked mode as defined in the previous section.

This logic differs slightly from the logic used with the PG\&E dataset due to the mode variable, which differentiates parked state from PTO operation.

\subsubsection{PTO Characterization}

For the PG\&E vehicles, PTO use is defined for the AT vehicles as times where vehicle speed is zero and engine speed is greater than 800 RPM. For the AM vehicles, PTO use is defined as times where the vehicle is not moving and the engine speed is greater than 850 RPM. PTO-ready mode for the AM trucks was set to be time when engine speed is between 850 and 1,050 RPM. "PTO-ready" corresponds to the vehicle being set to PTO mode but not performing any PTO work. The PTO-in-use mode was set to the time when the vehicle is not moving and the engine speed is greater than 1,050 RPM. The overall PTO state is a combination of the PTO-ready and the PTO-in-use modes. Figure 3 illustrates the different operating modes identified for a sample vehicle in the PG\&E dataset. One can see that by performing a peak detection analysis, each of the states and corresponding RPM values can be identified and visualized.

For the Odyne dataset, inclusion of the mode identifier simplified the analysis. In this case, time spent when the vehicle is in "Operate" mode is considered PTO time, as well as time when the 
vehicle is parked and the engine is operating at RPM ranges in excess of 1,000 RPM, which is very similar to the values identified and applied for the PG\&E dataset.
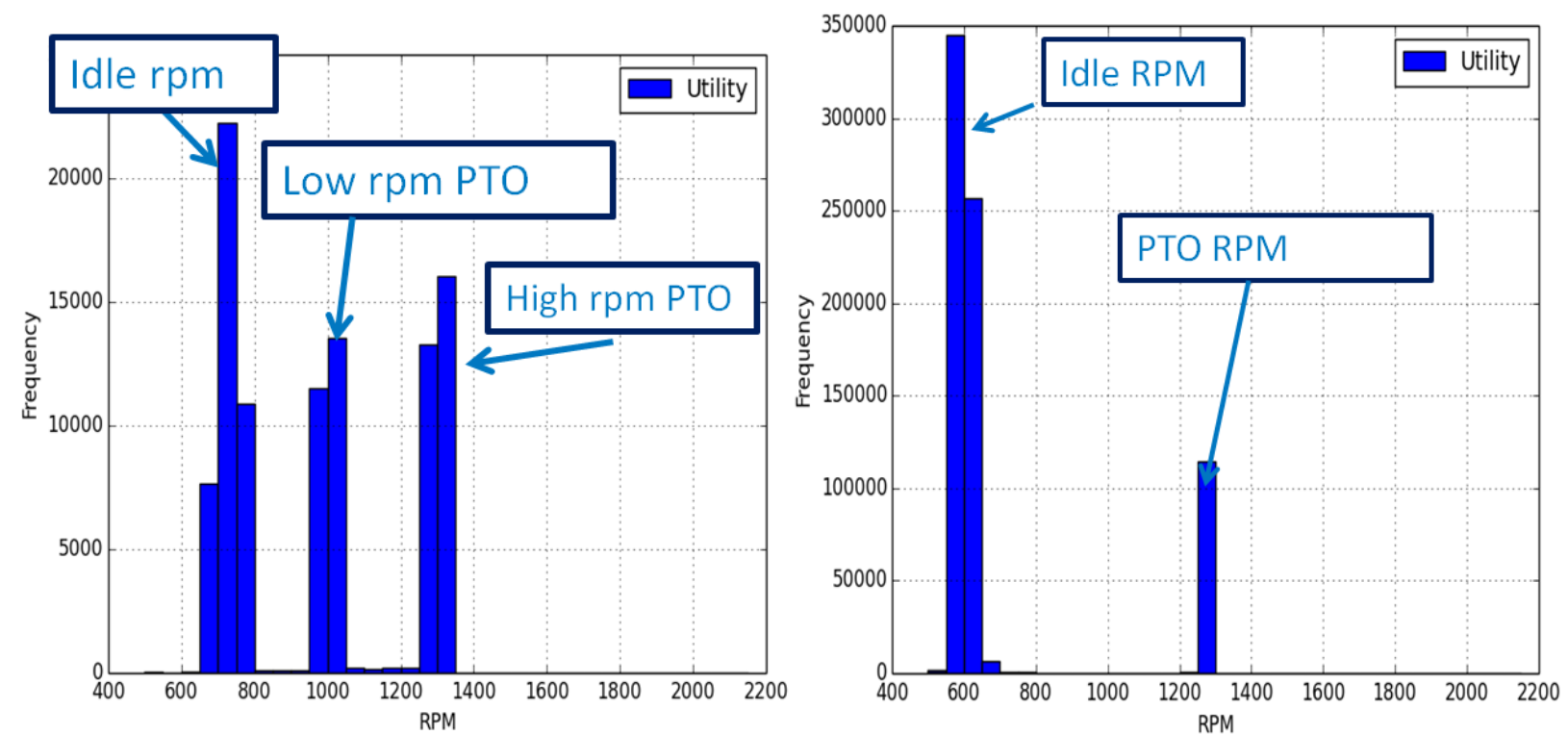

Figure 3. Illustration of the RPM method, histogram of engine RPM where vehicle speed is zero, but engine speed is greater than zero.

The distinct operation modes for the AT are on the left and for the AM are on the right.

\subsection{PTO Channel Method for Characterizing PTO and Idle Operation}

The PTO channel method is an alternative analysis method that was explored in addition to the RPM determination method described above. This method uses the "PTOState" SAE J1939 channel as the sole source indicating whether or not a vehicle is operating under PTO conditions. This analysis was only performed on the PG\&E dataset as the Odyne dataset already integrated PTO state in its mode identifier.

\subsubsection{Idle Characterization}

The same technique for idle in the RPM method was used for the PTO channel method as there is not specific channel for second-by-second tracking of engine idle speed.

\subsubsection{PTO Characterization}

The "PTOState" channel was used for the PTO method; "PTOState" greater than zero but less than 20 is an indication of PTO enable operation, according to SAE J1939.

\subsection{Driving Mode}

The driving mode for all methods is defined as time where vehicle speed is greater than zero and engine speed is greater than zero. 


\section{Discussion and Results}

Using the logic sets described above, data recorded for each vehicle for each day of operation were processed, and the time spent driving, idling, and PTO operation were calculated. The engine RPM and the PTO channel methods were compared against each other for validation. The RPM method was also compared to engine control unit binned data when available, for validation purposes. Aggregated days were removed where the vehicle did not drive for at least six minutes and days where the logged data accounted for more than 24 hours of operating time. These days were removed as they are not representative of typical vehicle operation due to either insufficient or excessive operating time. The final combined results include operating data for 85 utility vehicles representing 1,570 days of operation.

It was observed that even with limited PTO channel data, the PTO channel method and the RPM method results are very similar. As seen in Figure 4, the Engine RPM method yielded an average daily PTO usage time of 1.51 hours with a standard deviation of 1.72 hours and the PTO channel yielded an average daily PTO usage time of 1.81 hours with a standard deviation of 2.03 hours.

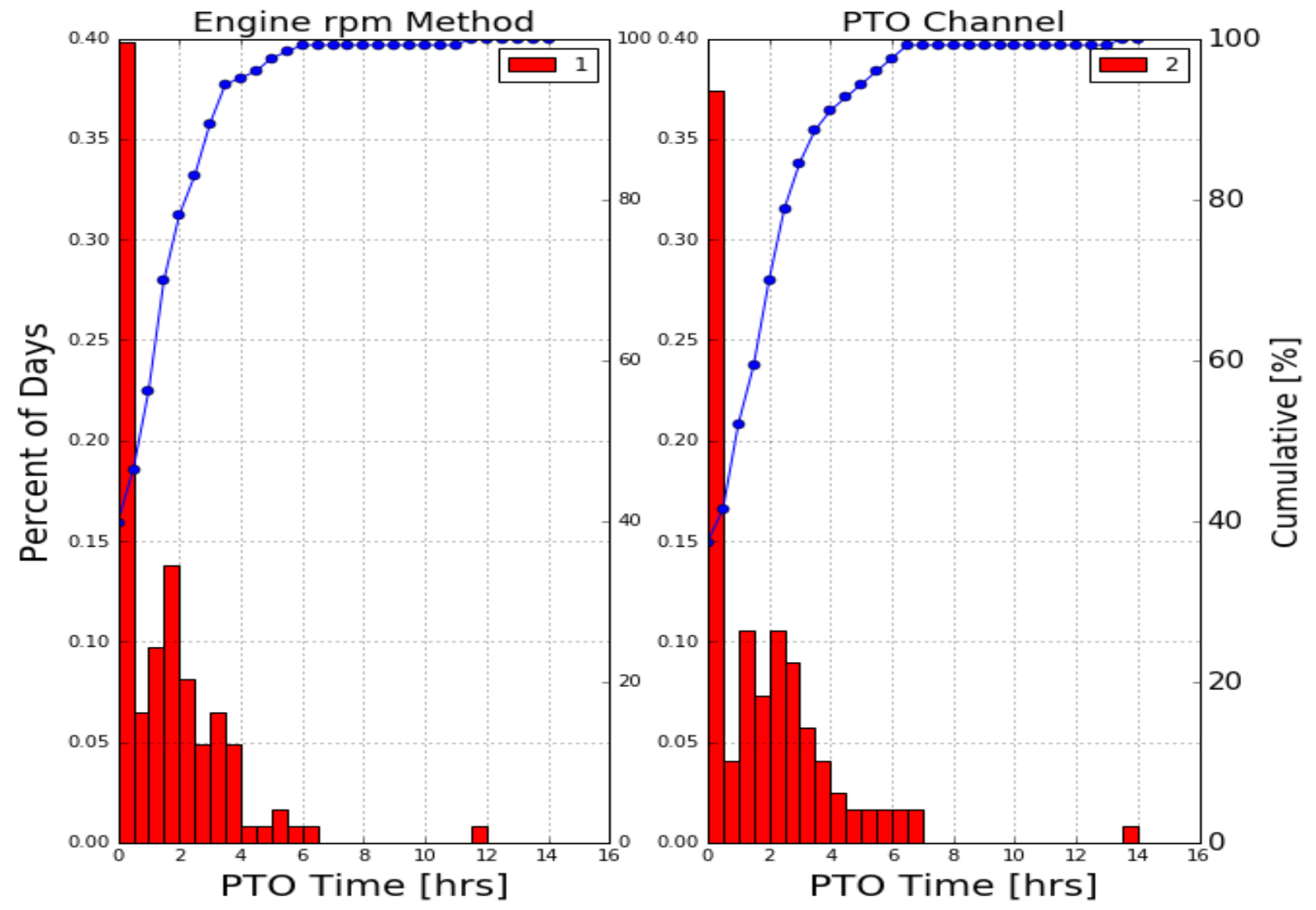

Figure 4. Comparison of PTO and engine RPM derived cumulative PTO operation for five PG\&E material handler trucks over 123 operating days.

The RPM method was applicable to most of the PTO-equipped vehicle data in the Fleet DNA database and was selected as the preferred method of analysis. In addition to application to a 
greater range of data, another reason for choosing the RPM method over the PTO channel method was due to the lack of reliability in the PTO signal; the PTO channel would either not report data or reported insufficient quality data for analysis. The RPM method, however, could always be applied to the Fleet DNA data as long as reliable engine speed signal data were collected at a sufficient frequency, which is necessary for this type of analysis. It is also worth mentioning that the data from PG\&E utility vehicles were collected by the NREL Fleet Testing and Evaluation team whereas the data for the Odyne hybrid systems were provided by the Electric Power Research Institute as explained in previous sections. Odyne. In both cases, data was logged at $1 \mathrm{~Hz}$. The RPM method was able to solve some challenges associated with data collection from multiple sources. Due to the methodology, the RPM method could be applied across data sources as long as initial work to identify appropriate RPM ranges was performed on each dataset.

The results of the analysis using the RPM method applied to the PG\&E and Odyne datasets were aggregated to create a unique utilization factor curve describing the PTO use in aggregate vs. total vehicle operating time. Figure 5 shows the aggregated statistics for all vehicles showing the proportions of time spent and fuel used for each of the operational modes - driving, idle, and PTO. Fuel used was determined from the SAE J1939 CAN-reported fuel rate data. Driving mode accounted for $34 \%$ of the operation time but $79 \%$ of the fuel used. PTO operation was about $37 \%$ and used $11 \%$ of the fuel. Idle mode represented $30 \%$ of the time for $10 \%$ of the fuel.
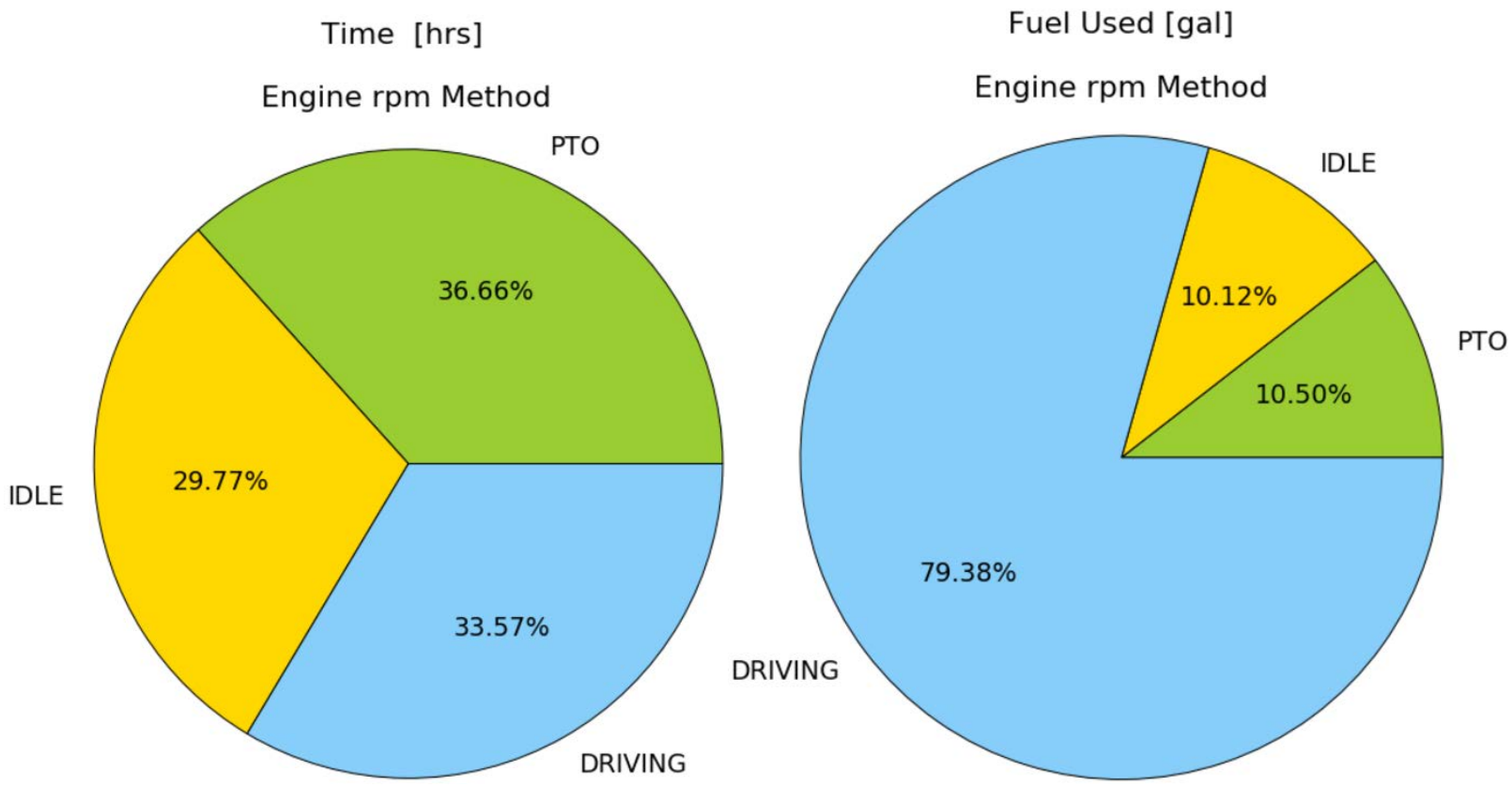

Figure 5. Percent of time [left] and fuel [right] spent in each operating mode

Figure 6 shows the resultant PTO utilization curves for the entire set of vehicles (PG\&E and Odyne combined) as a distribution of the hours of PTO operation by the number of days and the percentage of total days in the data. The data were also provided as an attached spreadsheet with the hours of driving time, idle time, and PTO operation time for each of the 1,570 days of operation. Figure 7 shows similar utilization curves for idle time. 

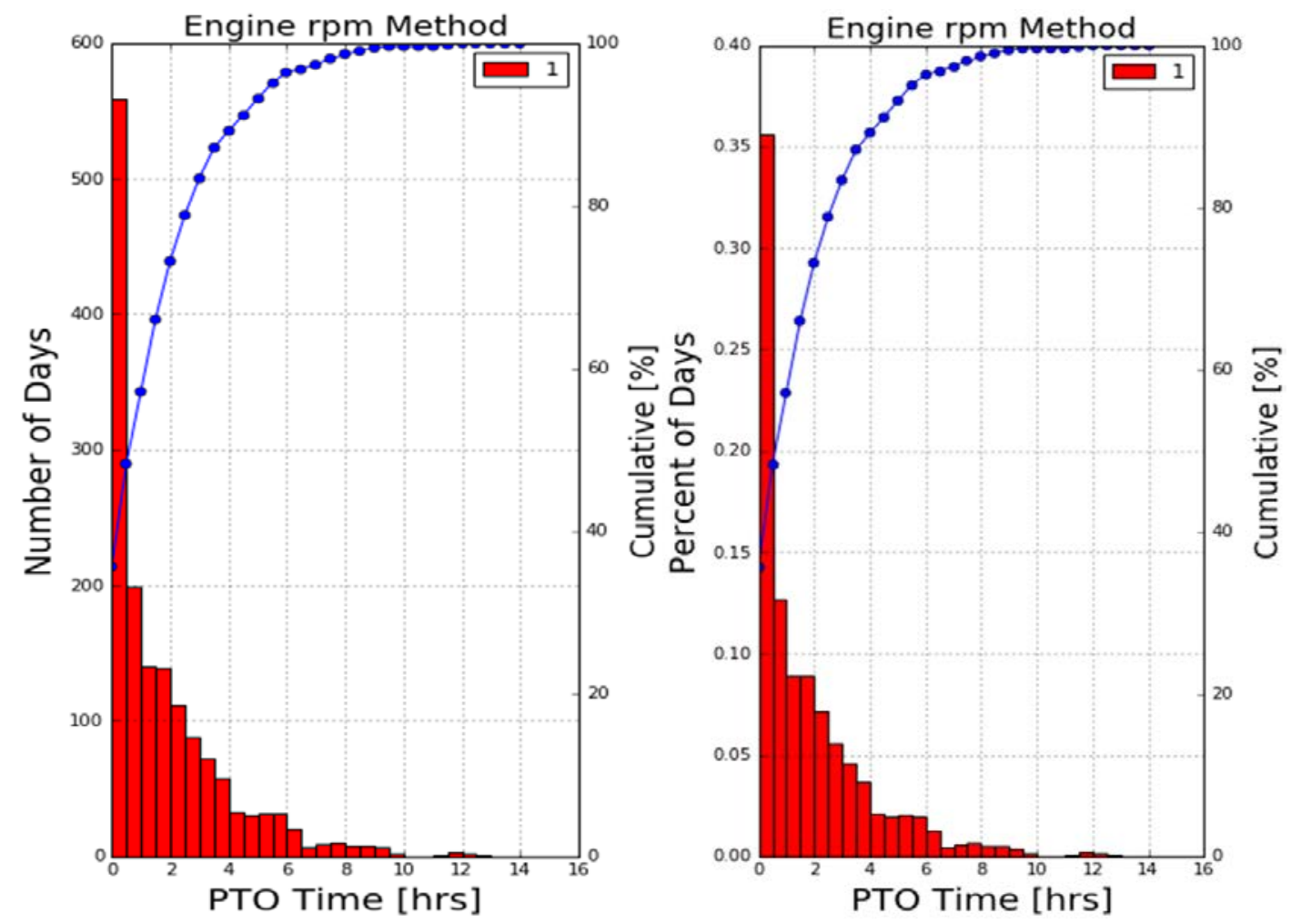

Figure 6. Distribution of PTO time for all vehicles in this study (PG\&E and Odyne combined)
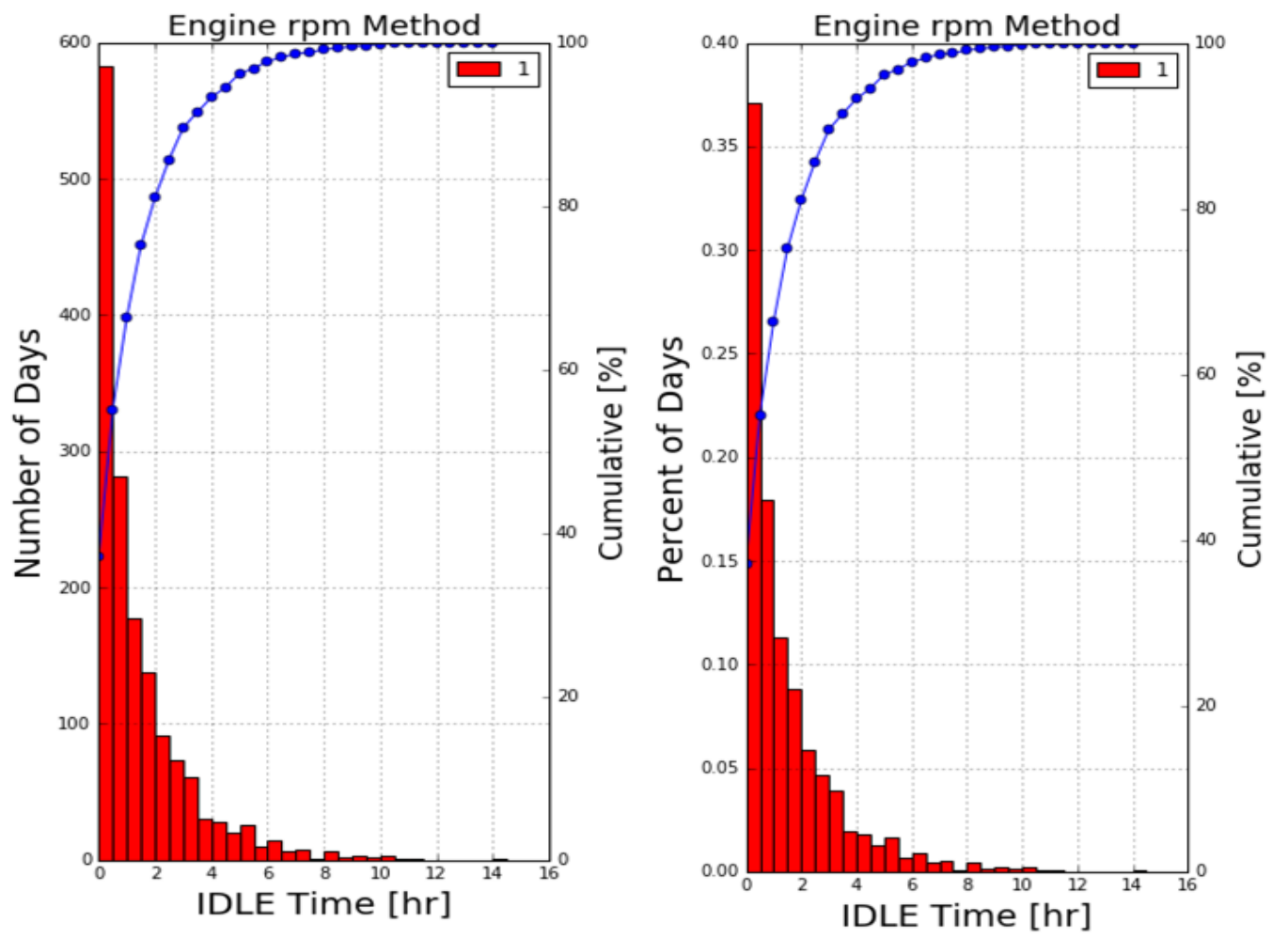

Figure 7. Distribution of idle time for all vehicles in this study (PG\&E and Odyne combined) 


\section{Conclusion}

The analysis described in this report resulted in the development of a utilization factor curve for PTO operation and idle time for a range of aerial lift utility trucks operating in the real world captured as part of two distinct data collection efforts. The analysis was conducted on data from 406 vehicle-days of PG\&E vehicle operation in California and 1,154 vehicle-days of operation from Odyne vehicles across several states. The researchers found that the RPM method is the preferred approach for analyzing PTO and idle behavior of the two methods examined in this report, as it is based on historical data mining techniques and demonstrated a greater level of flexibility and applicability when compared to the PTO method. This method provides aggregate operating statistics from various sources, types, and classes of vehicles. This approach gives insights on engine operation, and works very well with PTO equipped vehicles that have distinct operating modes. While the driving mode of utility vehicles does not overlap either with the idle mode or PTO mode, as is sometimes the case with PTO channel data., the engine RPM method might not be well suited for other applications such as refuse collection or concrete mixing trucks, as they typically utilize PTO while the vehicle is driving. In this case, the RPM method will not be able to separate increased speed due to PTO demands from traction power demands.

Another PTO operation that the RPM method might not be able to accurately capture is when the driver uses the PTO switch as a means to bypass an idle shut-down timer. In this case, the driver may set the engine PTO RPM at the engine idle RPM.

This study aggregated operation time from very different vehicles classes and types to come up with one utilization factor to characterize them. This approach, although it is valid and yields a good estimation of the overall bulk utilization breakdown, currently lacks the ability to give details on the utilization by vehicle class and type breakdown from one class and type of vehicle to another.

We saw for example that the class 5 utility vehicles from PG\&E mostly assigned to "troublemen" who respond to a variety of local grid issues spend more time driving and less extended PTO work, but do idle their vehicles a considerable amount of time whereas the class 8 material handlers were deployed to perform extensive heavy-duty PTO work; when combined, these differences are difficult to extract.

\section{Future Applications}

NREL researchers believe that the RPM method gives us the ability to look at details of a vehicle's behavior on a case by case basis while still offering the possibility for large batch-run processing. Researchers are interested in utilizing this technique in the future to look at emission rates based on the vehicle's engine speed in specifically defined operating modes instead of looking solely at the vehicle's speed. 


\section{References}

[1] Walkowicz, K., K. Kelly, A. Duran, and E. Burton. (2014). Fleet DNA Project Data. National Renewable Energy Laboratory. http://www.nrel.gov/fleetdna

[2] Kosowski, M., J. Dunckley, and D. Bowermaster. (2016). Results of Plug-In Hybrid Medium-Duty Truck Demonstration and Evaluation Program, 1-12. 


\section{Appendix B: PG\&E Vehicle Specifications}

\begin{tabular}{|l|l|}
\hline \multicolumn{2}{|l|}{ Altec AM JEMS Trucks (Material Handler) } \\
\hline Make & International \\
\hline Model & WorkStar 7500 \\
\hline GVWR & $56,000 \mathrm{lbs}$. \\
\hline Engine Power & $300 \mathrm{hp}$ \\
\hline Torque & $860 \mathrm{ft} .-\mathrm{lb}$. \\
\hline Transmission & Allison 3500 5-Speed \\
\hline Drive & $6 \times 6$ \\
\hline JEMS Battery Capacity & $18 \mathrm{kWh}$ \\
\hline
\end{tabular}

\begin{tabular}{|l|l|}
\hline \multicolumn{2}{|l|}{ Altec AT JEMS Trucks (Trouble Trucks) } \\
\hline Make & Ford \\
\hline Model & F-550 \\
\hline GWWR & $19,500 \mathrm{lbs}$. \\
\hline Engine Power & $300 \mathrm{hp}$ \\
\hline Torque & $660 \mathrm{ft} .-\mathrm{lb}$. \\
\hline Transmission & $6-S p e e d$ Automatic \\
\hline Drive & $4 \times 4$ \\
\hline JEMS Battery Capacity & $8 \mathrm{kWh}$ \\
\hline
\end{tabular}

\section{Source}

1. http://www.altec.com/site/uploads/Green_Fleet_literature.pdf 


\section{Appendix B: PG\&E Data Channels}

\begin{tabular}{|l|}
\hline Conventional Altec Trouble Truck (AT) \\
\hline Date \\
\hline time \\
\hline BoxT \\
\hline BoxV \\
\hline Acc_Lat \\
\hline Acc_Long \\
\hline Acc_Vert \\
\hline gps_Time \\
\hline gps_Lat \\
\hline gps_Long \\
\hline gps_NbSatellite \\
\hline gps_Altitude \\
\hline gps_Speed \\
\hline LOAD_PCT \\
\hline COOLANT_TEMP \\
\hline MAP \\
\hline EngSpeed \\
\hline WheelBasedVehicleSpeed \\
\hline IAT \\
\hline MAF \\
\hline THROTTLE_POS \\
\hline PTO_Status \\
\hline Time_Since_Engine_Start \\
\hline Fuel_Rail_Pressure_diesel_and_di \\
\hline Commanded_EGR \\
\hline EGR_Error \\
\hline Barometric_Pressure \\
\hline CATTEMP11 \\
\hline Relative_Throttle_Position \\
\hline Ambient_Air_Temperature \\
\hline Accelerator_Pedal_Position_D \\
\hline Accelerator_Pedal_Position_E \\
\hline Commanded_Throttle_Actuator_Control \\
\hline
\end{tabular}




\begin{tabular}{|l|}
\hline Conventional Altec Trouble Truck (AT) \\
\hline Minutes_Run_with_MIL_Activated \\
\hline Relative_APP \\
\hline FUEL_TIMING \\
\hline EngFuelRate \\
\hline EngPercentTorque_DD \\
\hline EngPercentTorque_ACT \\
\hline EngReferenceTorque \\
\hline OBDEngineDTC001 \\
\hline
\end{tabular}

\begin{tabular}{|l|l|}
\hline \multicolumn{2}{|l|}{ Conventional Altec Material Handler } \\
\hline Date & EnggmntSttsPTOEngAccssoryDrive1 \\
\hline time & EnggmntSttsPTOEngAccssoryDrive2 \\
\hline BoxT & AtLeastOnePTOEngaged \\
\hline BoxV & Aftrtrtmnt1DsIPrtcltFItrInt_0001 \\
\hline Acc_Lat & Aftrtrtmnt1DsIPrtcltFItrDffPrss \\
\hline Acc_Long & Aftrtrtmnt1PrtcltTrpOtItGasTemp \\
\hline Acc_Vert & Aftertreatment1ExhaustGasTemp1 \\
\hline gps_Time & Aftrtrtmnt1DsIPrtcltFItrInt_0000 \\
\hline gps_Lat & EngExhaustGasTempRightManifold \\
\hline gps_Long & EngExhaustGasTempLeftManifold \\
\hline gps_Quality & Aftrtrtmnt1SCRCatalystTankLevel \\
\hline gps_NbSatellite & EngExhaustGasRecirculation1Temp \\
\hline gps_Altitude & PneumaticSupplyPress \\
\hline gps_Speed & AirCompressorStatus \\
\hline TransOutputShaftSpeed_1 & EstPercentFanSpeed \\
\hline TransInputShaftSpeed_1 & FanDriveState \\
\hline AccelPedalPos1 & FanSpeed \\
\hline EngPercentLoadAtCurrentSpeed & HghRslutionTotalVehicleDistance \\
\hline ActMaxAvailEngPercentTorque & ProtectLampStatus_1 \\
\hline EngTorqueMode & AmberWarningLampStatus_1 \\
\hline DriversDemandEngPercentTorque & RedStopLampState_1 \\
\hline ActualEngPercentTorque & ProtectLampStatus_2 \\
\hline EngSpeed & AmberWarningLampStatus_2 \\
\hline TransSelectedGear_1 & RedStopLampState_2 \\
\hline & \\
\hline
\end{tabular}




\begin{tabular}{|c|c|}
\hline \multicolumn{2}{|l|}{ Conventional Altec Material Handler } \\
\hline TransCurrentGear_1 & EngTotalldleFuelUsed \\
\hline EngExhstGsRcrcltionMassFlowRate & EngTotalldleHours \\
\hline EngInletAirMassFlowRate & NominalFrictionPercentTorque \\
\hline Aftertreatment1IntakeNOx & EstEngPrsticLossesPercentTorque \\
\hline Aftertreatment 1 IntakeO2 & ExhaustGasMass \\
\hline Aftertreatment1OutletNOx & EngSpeedAtldlePoint1 \\
\hline Aftertreatment1OutletO2 & EngPercentTorqueAtldlePoint1 \\
\hline EngProtectLampData & EngSpeedAtPoint2 \\
\hline EngAmberWarningLampData & EngPercentTorqueAtPoint2 \\
\hline EngRedStopLampData & EngSpeedAtPoint3 \\
\hline Aftrtrtmnt2DsIOxdtnCtlystDffPrss & EngPercentTorqueAtPoint3 \\
\hline Aftrtrtmnt2DsIOxdtnCtlystlntkGsT & EngSpeedAtPoint4 \\
\hline Aftrtrtmnt2DsIOxdtnCtlystOtItGsT & EngPercentTorqueAtPoint4 \\
\hline Aftrtrtmnt1DsIOxdtnCtlystDffPrss & EngSpeedAtPoint5 \\
\hline Aftrtrtmnt1DsIOxdtnCtlystlntkGsT & EngPercentTorqueAtPoint5 \\
\hline Aftrtrtmnt1DsIOxdtnCtlystOtItGsT & EngSpeedAtHighldlePoint6 \\
\hline Aftrtrtmnt1SCRCtlystIntkGasTemp & EngGainOfEndspeedGovernor \\
\hline Aftrtrtmnt1SCRCtlystOtltGasTemp & EngReferenceTorque \\
\hline Aftrtrtmnt1SCRCtlystExhstGsDffPr & EngTotalHoursOfOperation \\
\hline Aftrtrtmnt1DsIExhstFIdTnkLwLvlln & EngTotalRevolutions \\
\hline DsIPrtcltFilter1SootLoadPercent & EngTripFuel \\
\hline DsIPrtclteFilter1AshLoadPercent & EngTotalFuelUsed \\
\hline DsIPrtcltFItr1TmSncLstActvRgnrtn & EngCoolantTemp \\
\hline DieselParticulateFilterLampCmd & EngFuelTemp \\
\hline DsIPrtcltFltrPssvRgnrtionStatus & EngOilTemp1 \\
\hline DsIPrtcltFltrActvRgnrtionStatus & EngIntercoolerTemp \\
\hline DieselParticulateFilterStatus & EngFuelDeliveryPress \\
\hline ExhaustSystemHighTempLampCmd & EngOilPress \\
\hline DsIPrtcltFltrActvRgnrtnFrcdStts & PowerTakeoffSetSpeed \\
\hline Aftertreatment1FuelRate & EngPTOGovernorEnableSwitch \\
\hline EnbISwtchTrnsfrCsOutputShaftPTO & EngPTOGovernorSetSwitch \\
\hline EnableSwitchTransOutputShaftPTO & ParkingBrakeSwitch \\
\hline EnableSwitchTransInputShaftPTO2 & WheelBasedVehicleSpeed \\
\hline EnableSwitchTransInputShaftPTO1 & BrakeSwitch \\
\hline
\end{tabular}




\begin{tabular}{|l|l|}
\hline \multicolumn{2}{|l|}{ Conventional Altec Material Handler } \\
\hline EnableSwitchPTOEngFlywheel & PTOState \\
\hline EnblSwitchPTOEngAccessoryDrive1 & EngFueIRate \\
\hline EnblSwitchPTOEngAccessoryDrive2 & BarometricPress \\
\hline EnggmntSttsTrnsfrCsOtptShaftPTO & AmbientAirTemp \\
\hline EnggmntSttusTransOutputShaftPTO & EngAirInletTemp \\
\hline EnggmntSttusTransInputShaftPTO2 & EngTurboBoostPress \\
\hline EnggmntSttusTransInputShaftPTO1 & EngIntakeManifold1Temp \\
\hline EngagementStatusPTOEngFlywheel & EngAirInletPress \\
\hline EngFueIRate_1 & EngExhaustGasTemp \\
\hline EngineSpeed & PTOControlSwitch \\
\hline WheelSpeedABSAxle1Left & PTOMode \\
\hline WheelSpeedABSAxle1Right & MassFlowRate \\
\hline TotalVehicleDistance & BatteryPotential \\
\hline
\end{tabular}




\section{Appendix C: Odyne Vehicle Specifications}

Vehicle configuration details for all 119 Odyne hybrid vehicles NREL received data on that were partially funded by the American Recovery and Reinvestment Act (ARRA). Eighty-six of the 119 vehicles $(72 \%)$ were configured as aerial/bucket trucks.

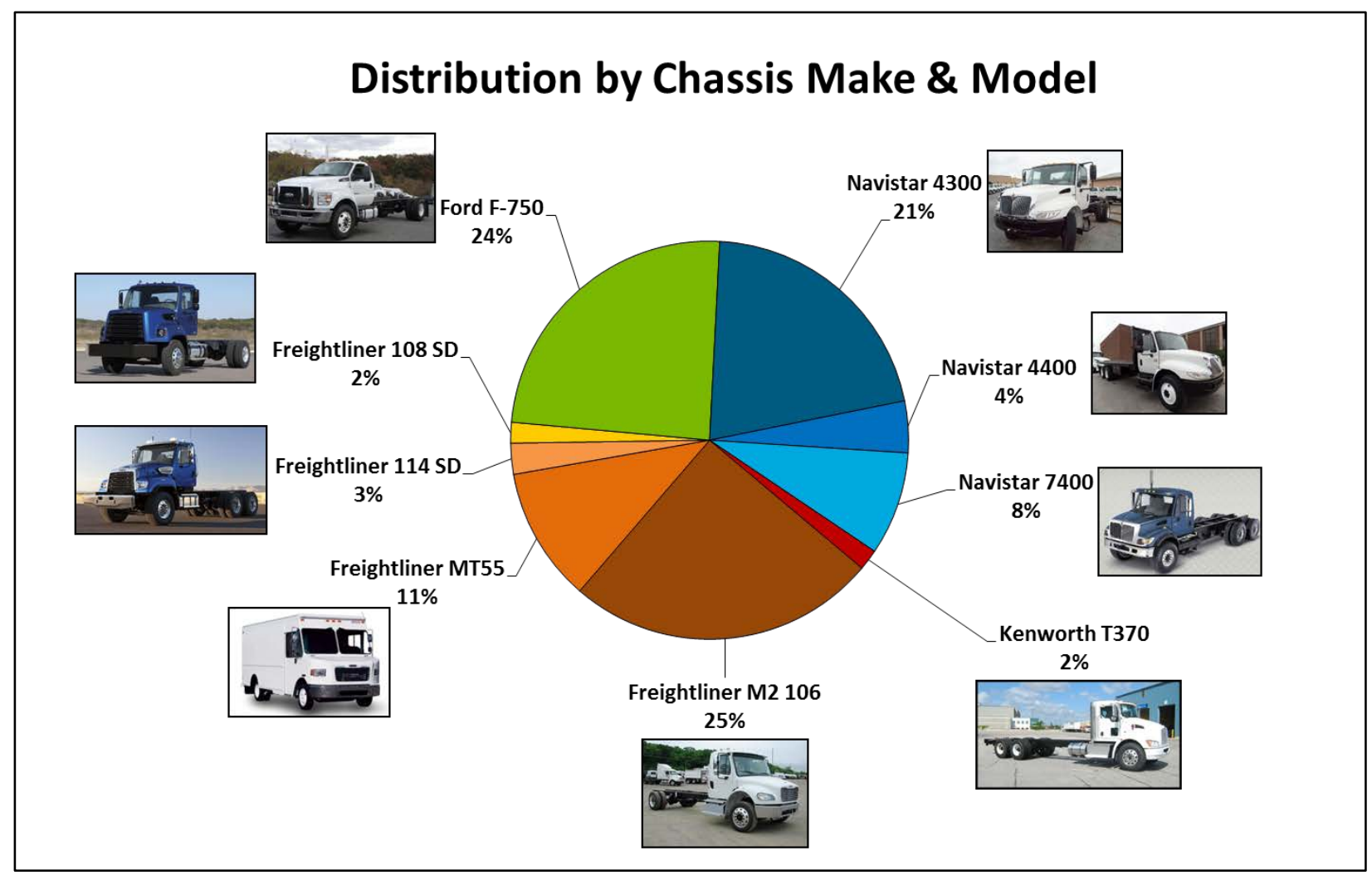

\begin{tabular}{|l|l|l|}
\hline Chassis Manufacturer & Chassis Model & Engine Models \\
\hline International & $4300 / 4400$ & DT, N9 \\
\hline International & $7300 / 7400 / 7500$ & DT, N9, N10, ISB6.7, ISL9 \\
\hline Kenworth & T370 & PX-9 \\
\hline Ford & F750 & ISB6.7 \\
\hline Freightliner & M2 106 & ISB6.7, ISL9 \\
\hline Freightliner & $108 S D$ & ISB6.7, ISL9 \\
\hline Freightliner & 114 SD & ISL9, DD13 \\
\hline Freightliner & MT55 & ISB6.7 \\
\hline
\end{tabular}




\begin{tabular}{|l|}
\hline $\begin{array}{l}\text { Aerial Vehicles (Bucket Trucks): } \\
\text { Service Body Manufacturers }\end{array}$ \\
\hline Altec \\
\hline Dueco \\
\hline Terex \\
\hline
\end{tabular}

\begin{tabular}{|l|l|}
\hline \multicolumn{2}{|l|}{ Additional Odyne System Specifications } \\
\hline Platform GVWR & $23,000-56,000$ pounds \\
\hline Motor Power & Peak: $95 \mathrm{hp}$ | Cont.: $56 \mathrm{hp}$ \\
\hline Motor Torque & $232 \mathrm{ft} .-$ lbs. \\
\hline Battery Capacity & 14.2 or $28.4 \mathrm{kWh}$ \\
\hline Transmission & Allison $2000,3000 \& 4000$ \\
\hline Drive & RWD 4 X2 or 6 X4 \\
\hline
\end{tabular}

Odyne Hybrid Systems architecture schematic:

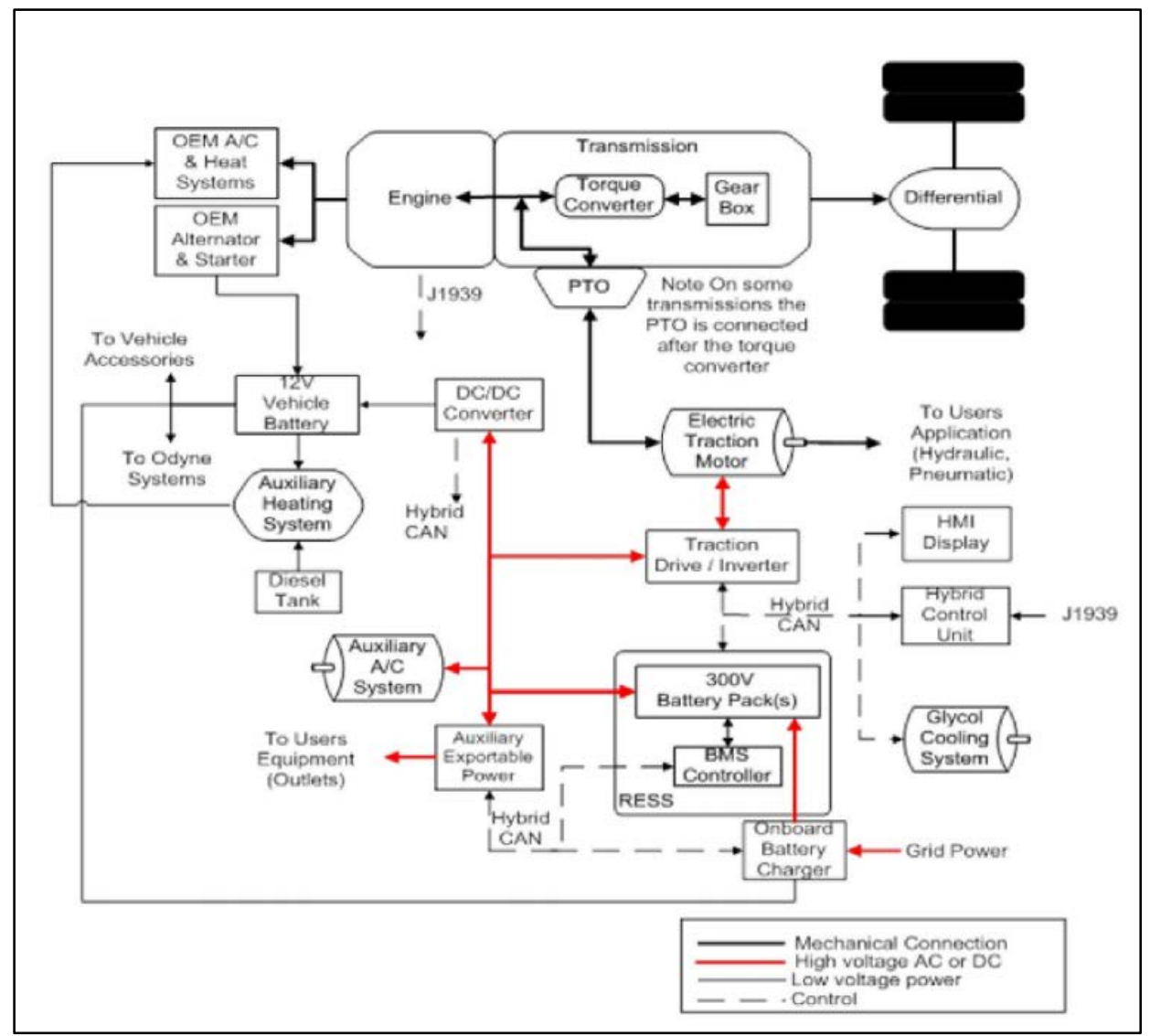




\section{Sources}

1. Odyne Hybrid Systems

2. http://www.osti.gov/scitech/servlets/purl/1234437 


\section{Appendix D: Odyne Hybrid Systems Data Channels}

\begin{tabular}{|l|}
\hline Odyne Data Channels \\
\hline Seconds elapsed beginning of month UTC \\
\hline Timestamp \\
\hline Accelerator Pedal Position \\
\hline Ambient Temperature \\
\hline Battery Current \\
\hline Battery Pack DC Power \\
\hline Battery Pack SOC \\
\hline Battery Voltage \\
\hline Brake Pedal Position Or Force \\
\hline Charger AC Current \\
\hline Charger AC Voltage \\
\hline Electric Air Conditioner State \\
\hline Engine Speed \\
\hline Export AC Current 1 \\
\hline Export AC Current 2 \\
\hline Export AC Voltage 1 \\
\hline Export AC Voltage 2 \\
\hline Fuel Flow Or Volume \\
\hline Odometer \\
\hline Operating Mode \\
\hline PTC Calibration \\
\hline PTC Firmware Version \\
\hline Vehicle Speed \\
\hline
\end{tabular}




\section{Appendix E: Final Data Dictionary}

\begin{tabular}{|l|l|}
\hline Header & Descriptions \\
\hline Day Index & A sequential numerical number to identify a vehicle day \\
\hline Driving Time $[\mathrm{hr}]$ & Time spent driving for that day \\
\hline Idle Time $[\mathrm{hr}]$ & Time spent while at idle for that day \\
\hline PTO Time $[\mathrm{hr}]$ & Time spent on PTO operation for that day \\
\hline Total & Total operating time of the day. It is the sum of all operating modes \\
\hline
\end{tabular}




\section{Appendix F: Final Data}

\begin{tabular}{|c|c|c|c|c|}
\hline $\begin{array}{l}\text { Day } \\
\text { Index }\end{array}$ & $\begin{array}{l}\text { Driving Time } \\
\text { [hr] }\end{array}$ & $\begin{array}{l}\text { Idle Time } \\
\text { [hr] }\end{array}$ & $\begin{array}{l}\text { PTO Time } \\
\text { [hr] }\end{array}$ & $\begin{array}{l}\text { Total } \\
\text { [hr] }\end{array}$ \\
\hline 0 & 0.9789 & 1.5142 & 2.1883 & 4.6814 \\
\hline 1 & 0.7461 & 0.2281 & 0.1628 & 1.1369 \\
\hline 2 & 0.7853 & 0.1836 & 0.0017 & 0.9706 \\
\hline 3 & 2.8008 & 0.5906 & 0.0289 & 3.4203 \\
\hline 4 & 0.9289 & 0.3833 & 0.0069 & 1.3192 \\
\hline 5 & 0.8386 & 0.3422 & 0.0031 & 1.1839 \\
\hline 6 & 1.8353 & 0.6267 & 0.4258 & 2.8878 \\
\hline 7 & 1.0167 & 0.2378 & 0.0694 & 1.3239 \\
\hline 8 & 1.0089 & 0.8625 & 1.4094 & 3.2808 \\
\hline 9 & 2.5014 & 1.1944 & 1.5658 & 5.2617 \\
\hline 10 & 0.6006 & 1.3533 & 4.1856 & 6.1394 \\
\hline 11 & 0.7217 & 0.0753 & 0.0103 & 0.8072 \\
\hline 12 & 0.2919 & 1.5681 & 2.8453 & 4.7053 \\
\hline 13 & 0.6197 & 0.6050 & 0.8503 & 2.0750 \\
\hline 14 & 1.0911 & 0.3864 & 0.3622 & 1.8397 \\
\hline 15 & 0.7875 & 0.3019 & 0.0208 & 1.1103 \\
\hline 16 & 0.8033 & 0.5078 & 0.8633 & 2.1744 \\
\hline 17 & 0.7228 & 1.1858 & 2.0639 & 3.9725 \\
\hline 18 & 1.3656 & 0.5094 & 0.0186 & 1.8936 \\
\hline 19 & 0.8225 & 0.2614 & 0.4956 & 1.5794 \\
\hline 20 & 1.9708 & 1.0569 & 0.9319 & 3.9597 \\
\hline 21 & 0.9336 & 0.4178 & 0.0228 & 1.3742 \\
\hline 22 & 0.6061 & 0.2872 & 0.0033 & 0.8967 \\
\hline 23 & 1.2078 & 0.9897 & 2.0372 & 4.2347 \\
\hline 24 & 0.4803 & 0.7839 & 1.5644 & 2.8286 \\
\hline 25 & 1.2097 & 0.9917 & 1.1622 & 3.3636 \\
\hline 26 & 0.5408 & 0.2958 & 0.0025 & 0.8392 \\
\hline 27 & 0.9919 & 1.2114 & 1.9256 & 4.1289 \\
\hline 28 & 1.5800 & 0.9600 & 1.0739 & 3.6139 \\
\hline 29 & 0.5511 & 1.2025 & 1.7314 & 3.4850 \\
\hline 30 & 0.4606 & 1.5306 & 3.9989 & 5.9900 \\
\hline 31 & 2.3742 & 1.8244 & 1.8747 & 6.0733 \\
\hline
\end{tabular}




\begin{tabular}{|c|c|c|c|c|}
\hline $\begin{array}{l}\text { Day } \\
\text { Index }\end{array}$ & $\begin{array}{l}\text { Driving Time } \\
\text { [hr] }\end{array}$ & $\begin{array}{l}\text { Idle Time } \\
\text { [hr] }\end{array}$ & $\begin{array}{l}\text { PTO Time } \\
\text { [hr] }\end{array}$ & $\begin{array}{l}\text { Total } \\
\text { [hr] }\end{array}$ \\
\hline 32 & 2.6867 & 1.0114 & 2.2908 & 5.9889 \\
\hline 33 & 1.8694 & 1.4506 & 2.0081 & 5.3281 \\
\hline 34 & 2.4564 & 1.9619 & 1.8817 & 6.3000 \\
\hline 35 & 2.0936 & 0.9206 & 1.5497 & 4.5639 \\
\hline 36 & 2.1997 & 0.8608 & 1.8033 & 4.8639 \\
\hline 37 & 2.6781 & 0.8700 & 1.4636 & 5.0117 \\
\hline 38 & 3.3800 & 1.1931 & 0.7694 & 5.3425 \\
\hline 39 & 3.8347 & 2.7939 & 3.4894 & 10.1181 \\
\hline 40 & 1.3417 & 1.3244 & 2.6092 & 5.2753 \\
\hline 41 & 1.5164 & 0.7433 & 1.3894 & 3.6492 \\
\hline 42 & 2.4928 & 1.3133 & 1.5806 & 5.3867 \\
\hline 43 & 1.4069 & 1.0742 & 1.6239 & 4.1050 \\
\hline 44 & 3.0897 & 1.8147 & 3.1317 & 8.0361 \\
\hline 45 & 1.5133 & 1.7133 & 1.9589 & 5.1856 \\
\hline 46 & 0.8200 & 0.4603 & 0.9808 & 2.2611 \\
\hline 47 & 2.0125 & 0.5389 & 0.7375 & 3.2889 \\
\hline 48 & 3.1003 & 0.2047 & 0.0022 & 3.3072 \\
\hline 49 & 0.1347 & 0.1194 & 0.0003 & 0.2544 \\
\hline 50 & 4.2578 & 3.3539 & 11.5111 & 19.1228 \\
\hline 51 & 0.1014 & 0.6861 & 0.0194 & 0.8069 \\
\hline 52 & 2.0722 & 1.7172 & 5.8422 & 9.6317 \\
\hline 53 & 1.6267 & 3.2858 & 3.6814 & 8.5939 \\
\hline 54 & 1.8075 & 1.3517 & 3.8114 & 6.9706 \\
\hline 55 & 2.9806 & 0.3456 & 0.0122 & 3.3383 \\
\hline 56 & 2.8639 & 0.2675 & 0.0092 & 3.1406 \\
\hline 57 & 0.5992 & 0.4317 & 0.0189 & 1.0497 \\
\hline 58 & 0.5108 & 1.0106 & 3.1661 & 4.6875 \\
\hline 59 & 2.2717 & 0.4642 & 0.0186 & 2.7544 \\
\hline 60 & 1.5625 & 1.6936 & 1.9397 & 5.1958 \\
\hline 61 & 1.2811 & 2.1192 & 4.8700 & 8.2703 \\
\hline 62 & 0.7942 & 0.7892 & 1.7828 & 3.3661 \\
\hline 63 & 1.0339 & 0.2542 & 0.4711 & 1.7592 \\
\hline 64 & 0.9300 & 0.7450 & 0.9744 & 2.6494 \\
\hline 65 & 1.0450 & 1.3578 & 3.2681 & 5.6708 \\
\hline
\end{tabular}




\begin{tabular}{|c|c|c|c|c|}
\hline $\begin{array}{l}\text { Day } \\
\text { Index }\end{array}$ & $\begin{array}{l}\text { Driving Time } \\
\text { [hr] }\end{array}$ & $\begin{array}{l}\text { Idle Time } \\
\text { [hr] }\end{array}$ & $\begin{array}{l}\text { PTO Time } \\
\text { [hr] }\end{array}$ & $\begin{array}{l}\text { Total } \\
\text { [hr] }\end{array}$ \\
\hline 66 & 1.0200 & 0.5300 & 0.0136 & 1.5636 \\
\hline 67 & 0.4736 & 0.5014 & 0.0297 & 1.0047 \\
\hline 68 & 0.7022 & 0.7358 & 0.7886 & 2.2267 \\
\hline 69 & 0.1694 & 0.3250 & 0.0289 & 0.5233 \\
\hline 70 & 2.0181 & 0.9878 & 2.6497 & 5.6556 \\
\hline 71 & 0.2978 & 1.1772 & 3.6664 & 5.1414 \\
\hline 72 & 0.4175 & 0.8172 & 2.5031 & 3.7378 \\
\hline 73 & 1.7856 & 0.9950 & 2.9936 & 5.7742 \\
\hline 74 & 0.8547 & 1.4686 & 3.6517 & 5.9750 \\
\hline 75 & 0.9531 & 0.8561 & 2.4914 & 4.3006 \\
\hline 76 & 1.7047 & 0.3839 & 3.5714 & 5.6600 \\
\hline 77 & 1.0700 & 0.3586 & 1.7753 & 3.2039 \\
\hline 78 & 1.6111 & 0.2461 & 0.0022 & 1.8594 \\
\hline 79 & 4.0219 & 0.3683 & 0.0025 & 4.3928 \\
\hline 80 & 1.2547 & 0.6644 & 3.4019 & 5.3211 \\
\hline 81 & 1.0703 & 0.7744 & 1.1400 & 2.9847 \\
\hline 82 & 0.6147 & 0.3850 & 0.4219 & 1.4217 \\
\hline 83 & 2.9161 & 0.9228 & 0.1431 & 3.9819 \\
\hline 84 & 0.7275 & 0.4714 & 2.1281 & 3.3269 \\
\hline 85 & 0.2536 & 0.2883 & 3.1214 & 3.6633 \\
\hline 86 & 0.8239 & 0.2992 & 2.2544 & 3.3775 \\
\hline 87 & 1.0550 & 0.7214 & 3.3164 & 5.0928 \\
\hline 88 & 0.9781 & 0.8042 & 1.2439 & 3.0261 \\
\hline 89 & 0.2981 & 0.2536 & 0.0550 & 0.6067 \\
\hline 90 & 0.3572 & 0.4786 & 0.0078 & 0.8436 \\
\hline 91 & 0.7078 & 0.2614 & 0.0128 & 0.9819 \\
\hline 92 & 0.7011 & 0.4297 & 2.0842 & 3.2150 \\
\hline 93 & 0.8244 & 0.6289 & 0.0092 & 1.4625 \\
\hline 94 & 1.0283 & 0.4319 & 1.0086 & 2.4689 \\
\hline 95 & 1.2114 & 0.9469 & 5.4933 & 7.6517 \\
\hline 96 & 1.1558 & 0.7803 & 0.4639 & 2.4000 \\
\hline 97 & 1.5311 & 0.7622 & 1.9111 & 4.2044 \\
\hline 98 & 4.2500 & 0.3772 & 0.0106 & 4.6378 \\
\hline 99 & 4.1058 & 0.3011 & 6.2456 & 10.6525 \\
\hline
\end{tabular}




\begin{tabular}{|c|c|c|c|c|}
\hline $\begin{array}{l}\text { Day } \\
\text { Index }\end{array}$ & $\begin{array}{l}\text { Driving Time } \\
\text { [hr] }\end{array}$ & $\begin{array}{l}\text { Idle Time } \\
\text { [hr] }\end{array}$ & $\begin{array}{l}\text { PTO Time } \\
\text { [hr] }\end{array}$ & $\begin{array}{l}\text { Total } \\
\text { [hr] }\end{array}$ \\
\hline 100 & 0.5839 & 0.4075 & 1.0639 & 2.0553 \\
\hline 101 & 1.3814 & 0.5358 & 0.0161 & 1.9333 \\
\hline 102 & 1.4389 & 0.4506 & 1.7397 & 3.6292 \\
\hline 103 & 1.4267 & 0.5772 & 1.9672 & 3.9711 \\
\hline 104 & 4.1561 & 1.0236 & 0.4633 & 5.6431 \\
\hline 105 & 1.5950 & 0.3767 & 2.5742 & 4.5458 \\
\hline 106 & 1.7811 & 0.6556 & 5.3700 & 7.8067 \\
\hline 107 & 1.4964 & 0.2078 & 1.3933 & 3.0975 \\
\hline 108 & 0.1936 & 0.2222 & 3.0114 & 3.4272 \\
\hline 109 & 3.3189 & 0.1925 & 1.3467 & 4.8581 \\
\hline 110 & 1.5581 & 0.2178 & 0.0217 & 1.7975 \\
\hline 111 & 1.1725 & 1.0806 & 2.1353 & 4.3883 \\
\hline 112 & 0.3564 & 0.4269 & 1.1419 & 1.9253 \\
\hline 113 & 6.4792 & 2.1969 & 1.2978 & 9.9739 \\
\hline 114 & 5.9672 & 3.0989 & 0.0303 & 9.0964 \\
\hline 115 & 8.1264 & 3.2167 & 0.1011 & 11.4442 \\
\hline 116 & 4.9639 & 0.9800 & 0.0025 & 5.9464 \\
\hline 117 & 4.4828 & 5.4100 & 2.6097 & 12.5025 \\
\hline 118 & 6.7989 & 2.9581 & 0.9481 & 10.7050 \\
\hline 119 & 5.0719 & 2.0928 & 0.5125 & 7.6772 \\
\hline 120 & 6.8306 & 2.3406 & 1.5833 & 10.7544 \\
\hline 121 & 6.5133 & 4.5408 & 0.7233 & 11.7775 \\
\hline 122 & 6.3592 & 5.6250 & 0.2428 & 12.2269 \\
\hline 123 & 7.5011 & 3.4486 & 0.0728 & 11.0225 \\
\hline 124 & 6.8614 & 3.3369 & 0.0350 & 10.2333 \\
\hline 125 & 5.9064 & 2.2214 & 0.0156 & 8.1433 \\
\hline 126 & 4.7511 & 4.3542 & 0.5736 & 9.6789 \\
\hline 127 & 6.9711 & 4.2075 & 1.2722 & 12.4508 \\
\hline 128 & 5.8183 & 5.8983 & 0.9919 & 12.7086 \\
\hline 129 & 4.1050 & 2.0875 & 0.0664 & 6.2589 \\
\hline 130 & 6.0522 & 1.0000 & 0.0097 & 7.0619 \\
\hline 131 & 8.2369 & 3.6067 & 0.7597 & 12.6033 \\
\hline 132 & 5.2606 & 2.1100 & 1.3292 & 8.6997 \\
\hline 133 & 3.5964 & 2.0506 & 0.1942 & 5.8411 \\
\hline
\end{tabular}




\begin{tabular}{|c|c|c|c|c|}
\hline $\begin{array}{l}\text { Day } \\
\text { Index }\end{array}$ & $\begin{array}{l}\text { Driving Time } \\
\text { [hr] }\end{array}$ & $\begin{array}{l}\text { Idle Time } \\
\text { [hr] }\end{array}$ & $\begin{array}{l}\text { PTO Time } \\
\text { [hr] }\end{array}$ & $\begin{array}{l}\text { Total } \\
\text { [hr] }\end{array}$ \\
\hline 134 & 3.3164 & 2.7761 & 0.0333 & 6.1258 \\
\hline 135 & 3.9886 & 1.7206 & 0.2528 & 5.9619 \\
\hline 136 & 1.8561 & 0.6961 & 1.3594 & 3.9117 \\
\hline 137 & 2.0078 & 0.5197 & 0.4661 & 2.9936 \\
\hline 138 & 2.0136 & 1.5383 & 0.1919 & 3.7439 \\
\hline 139 & 2.5056 & 1.0239 & 0.1081 & 3.6375 \\
\hline 140 & 2.3544 & 1.6056 & 0.9178 & 4.8778 \\
\hline 141 & 3.8708 & 4.0311 & 0.2222 & 8.1242 \\
\hline 142 & 2.4133 & 2.2442 & 0.0172 & 4.6747 \\
\hline 143 & 3.0492 & 1.4756 & 0.6183 & 5.1431 \\
\hline 144 & 3.9978 & 1.3928 & 0.6469 & 6.0375 \\
\hline 145 & 1.6767 & 1.0119 & 0.3231 & 3.0117 \\
\hline 146 & 3.2847 & 3.8414 & 1.1883 & 8.3144 \\
\hline 147 & 1.9331 & 0.8447 & 0.5264 & 3.3042 \\
\hline 148 & 3.2053 & 2.5564 & 0.1972 & 5.9589 \\
\hline 149 & 2.8811 & 1.5975 & 0.0078 & 4.4864 \\
\hline 150 & 3.0061 & 1.6944 & 0.2344 & 4.9350 \\
\hline 151 & 3.3431 & 2.3858 & 0.7394 & 6.4683 \\
\hline 152 & 0.7831 & 0.2114 & 0.0283 & 1.0228 \\
\hline 153 & 4.4719 & 5.2894 & 0.7961 & 10.5575 \\
\hline 154 & 2.4408 & 2.0328 & 0.4047 & 4.8783 \\
\hline 155 & 3.1878 & 9.0394 & 0.0469 & 12.2742 \\
\hline 156 & 2.7511 & 1.4842 & 0.1686 & 4.4039 \\
\hline 157 & 3.0436 & 2.2597 & 0.0339 & 5.3372 \\
\hline 158 & 1.5306 & 2.4225 & 0.0053 & 3.9583 \\
\hline 159 & 2.4511 & 2.4550 & 0.4228 & 5.3289 \\
\hline 160 & 2.6933 & 1.3725 & 0.0194 & 4.0853 \\
\hline 161 & 2.7264 & 2.1039 & 0.2269 & 5.0572 \\
\hline 162 & 2.6069 & 2.3817 & 0.2183 & 5.2069 \\
\hline 163 & 3.7289 & 0.8511 & 0.0558 & 4.6358 \\
\hline 164 & 3.0200 & 1.1764 & 1.1436 & 5.3400 \\
\hline 165 & 1.8961 & 0.8189 & 0.5047 & 3.2197 \\
\hline 166 & 2.4603 & 1.2244 & 0.9972 & 4.6819 \\
\hline 167 & 2.5764 & 0.7861 & 0.2658 & 3.6283 \\
\hline
\end{tabular}




\begin{tabular}{|c|c|c|c|c|}
\hline $\begin{array}{l}\text { Day } \\
\text { Index }\end{array}$ & $\begin{array}{l}\text { Driving Time } \\
\text { [hr] }\end{array}$ & $\begin{array}{l}\text { Idle Time } \\
\text { [hr] }\end{array}$ & $\begin{array}{l}\text { PTO Time } \\
\text { [hr] }\end{array}$ & $\begin{array}{l}\text { Total } \\
\text { [hr] }\end{array}$ \\
\hline 168 & 1.9919 & 1.8542 & 0.8631 & 4.7092 \\
\hline 169 & 3.3525 & 1.4872 & 0.3622 & 5.2019 \\
\hline 170 & 1.7650 & 0.9464 & 0.0189 & 2.7303 \\
\hline 171 & 2.4061 & 1.0253 & 0.5250 & 3.9564 \\
\hline 172 & 3.3997 & 1.6042 & 0.1892 & 5.1931 \\
\hline 173 & 3.1886 & 3.5308 & 1.7508 & 8.4703 \\
\hline 174 & 4.0331 & 2.0333 & 1.4381 & 7.5044 \\
\hline 175 & 2.3086 & 0.9569 & 0.2764 & 3.5419 \\
\hline 176 & 3.3075 & 2.8447 & 0.9186 & 7.0708 \\
\hline 177 & 2.3717 & 0.7583 & 0.0100 & 3.1400 \\
\hline 178 & 3.2031 & 1.9014 & 0.2756 & 5.3800 \\
\hline 179 & 3.4556 & 1.0669 & 0.1831 & 4.7056 \\
\hline 180 & 4.6786 & 2.6778 & 0.8069 & 8.1633 \\
\hline 181 & 3.0125 & 1.0414 & 1.3194 & 5.3733 \\
\hline 182 & 0.5325 & 0.0411 & 0.0467 & 0.6203 \\
\hline 183 & 3.5328 & 3.2489 & 0.0214 & 6.8031 \\
\hline 184 & 0.7108 & 0.1092 & 0.0419 & 0.8619 \\
\hline 185 & 2.8086 & 6.5661 & 0.9717 & 10.3464 \\
\hline 186 & 4.3364 & 7.4428 & 2.1050 & 13.8842 \\
\hline 187 & 2.6831 & 10.9856 & 2.0267 & 15.6953 \\
\hline 188 & 3.4517 & 9.9581 & 0.2608 & 13.6706 \\
\hline 189 & 6.2183 & 7.8581 & 0.4417 & 14.5181 \\
\hline 190 & 5.3681 & 8.6408 & 0.7444 & 14.7533 \\
\hline 191 & 4.7483 & 8.4758 & 0.0100 & 13.2342 \\
\hline 192 & 3.6219 & 1.6597 & 0.0347 & 5.3164 \\
\hline 193 & 2.0139 & 1.3569 & 0.0267 & 3.3975 \\
\hline 194 & 2.8289 & 8.1103 & 0.1303 & 11.0694 \\
\hline 195 & 4.9756 & 6.6453 & 0.2056 & 11.8264 \\
\hline 196 & 4.5600 & 11.1222 & 0.7756 & 16.4578 \\
\hline 197 & 6.3436 & 14.3539 & 0.8086 & 21.5061 \\
\hline 198 & 3.9589 & 3.8842 & 1.0903 & 8.9333 \\
\hline 199 & 5.2594 & 6.9144 & 0.3453 & 12.5192 \\
\hline 200 & 11.5608 & 10.4128 & 1.5286 & 23.5022 \\
\hline 201 & 1.2475 & 3.0542 & 1.1586 & 5.4603 \\
\hline
\end{tabular}




\begin{tabular}{|c|c|c|c|c|}
\hline $\begin{array}{l}\text { Day } \\
\text { Index }\end{array}$ & $\begin{array}{l}\text { Driving Time } \\
\text { [hr] }\end{array}$ & $\begin{array}{l}\text { Idle Time } \\
\text { [hr] }\end{array}$ & $\begin{array}{l}\text { PTO Time } \\
\text { [hr] }\end{array}$ & $\begin{array}{l}\text { Total } \\
\text { [hr] }\end{array}$ \\
\hline 202 & 2.8997 & 8.4161 & 0.3572 & 11.6731 \\
\hline 203 & 1.7764 & 6.0408 & 0.1000 & 7.9172 \\
\hline 204 & 1.5992 & 2.3836 & 0.1211 & 4.1039 \\
\hline 205 & 2.4833 & 4.6400 & 1.9111 & 9.0344 \\
\hline 206 & 1.9339 & 3.3439 & 0.5300 & 5.8078 \\
\hline 207 & 2.8786 & 4.7181 & 1.5581 & 9.1547 \\
\hline 208 & 1.8308 & 5.2150 & 1.4536 & 8.4994 \\
\hline 209 & 2.1092 & 3.6689 & 0.2983 & 6.0764 \\
\hline 210 & 0.9658 & 0.6381 & 0.2447 & 1.8486 \\
\hline 211 & 1.0083 & 0.5242 & 0.0278 & 1.5603 \\
\hline 212 & 2.0094 & 2.7983 & 0.6242 & 5.4319 \\
\hline 213 & 2.8514 & 3.2642 & 0.1994 & 6.3150 \\
\hline 214 & 0.9656 & 0.2722 & 0.0136 & 1.2514 \\
\hline 215 & 2.9364 & 3.3167 & 1.0158 & 7.2689 \\
\hline 216 & 1.4475 & 2.1264 & 0.1333 & 3.7072 \\
\hline 217 & 1.8544 & 3.8775 & 0.5094 & 6.2414 \\
\hline 218 & 1.9361 & 3.6369 & 0.5961 & 6.1692 \\
\hline 219 & 2.4364 & 4.0397 & 1.0228 & 7.4989 \\
\hline 220 & 3.4925 & 3.6675 & 1.3428 & 8.5028 \\
\hline 221 & 1.8078 & 4.4658 & 0.4319 & 6.7056 \\
\hline 222 & 1.7939 & 3.4094 & 1.2503 & 6.4536 \\
\hline 223 & 2.6264 & 1.0425 & 0.0300 & 3.6989 \\
\hline 224 & 4.5903 & 3.5269 & 0.4758 & 8.5931 \\
\hline 225 & 1.0000 & 2.2317 & 0.0311 & 3.2628 \\
\hline 226 & 3.6442 & 3.2136 & 0.4394 & 7.2972 \\
\hline 227 & 1.9411 & 3.2092 & 0.2447 & 5.3950 \\
\hline 228 & 1.4403 & 2.4756 & 0.1003 & 4.0161 \\
\hline 229 & 1.3197 & 2.5686 & 0.2800 & 4.1683 \\
\hline 230 & 3.1919 & 3.4675 & 1.9342 & 8.5936 \\
\hline 231 & 1.9283 & 1.1647 & 0.6647 & 3.7578 \\
\hline 232 & 2.7119 & 2.0014 & 0.2225 & 4.9358 \\
\hline 233 & 2.2989 & 1.6161 & 0.0394 & 3.9544 \\
\hline 234 & 4.3586 & 1.8828 & 0.4244 & 6.6658 \\
\hline 235 & 4.6378 & 8.5083 & 0.4131 & 13.5592 \\
\hline
\end{tabular}




\begin{tabular}{|c|c|c|c|c|}
\hline $\begin{array}{l}\text { Day } \\
\text { Index }\end{array}$ & $\begin{array}{l}\text { Driving Time } \\
\text { [hr] }\end{array}$ & $\begin{array}{l}\text { Idle Time } \\
\text { [hr] }\end{array}$ & $\begin{array}{l}\text { PTO Time } \\
\text { [hr] }\end{array}$ & $\begin{array}{l}\text { Total } \\
\text { [hr] }\end{array}$ \\
\hline 236 & 2.6664 & 2.2631 & 0.8669 & 5.7964 \\
\hline 237 & 4.2147 & 4.7567 & 0.6381 & 9.6094 \\
\hline 238 & 2.8786 & 2.9206 & 0.6878 & 6.4869 \\
\hline 239 & 2.5747 & 1.9747 & 0.7172 & 5.2667 \\
\hline 240 & 3.9758 & 3.8853 & 0.6728 & 8.5339 \\
\hline 241 & 3.8797 & 5.4753 & 0.7033 & 10.0583 \\
\hline 242 & 3.8944 & 5.5492 & 1.0306 & 10.4742 \\
\hline 243 & 3.3083 & 6.3883 & 0.3267 & 10.0233 \\
\hline 244 & 1.8153 & 1.5450 & 0.2503 & 3.6106 \\
\hline 245 & 3.1269 & 1.3869 & 0.0519 & 4.5658 \\
\hline 246 & 2.1572 & 5.3258 & 0.5842 & 8.0672 \\
\hline 247 & 4.4344 & 4.6978 & 0.4192 & 9.5514 \\
\hline 248 & 5.0200 & 5.3908 & 0.9447 & 11.3556 \\
\hline 249 & 2.7308 & 4.4281 & 0.5028 & 7.6617 \\
\hline 250 & 3.6944 & 3.0806 & 0.9350 & 7.7100 \\
\hline 251 & 3.6367 & 2.1078 & 0.8231 & 6.5675 \\
\hline 252 & 2.5450 & 1.8267 & 1.2736 & 5.6453 \\
\hline 253 & 2.5258 & 2.2639 & 0.8431 & 5.6328 \\
\hline 254 & 3.3517 & 9.4936 & 0.6572 & 13.5025 \\
\hline 255 & 3.7167 & 2.0708 & 0.2092 & 5.9967 \\
\hline 256 & 1.3889 & 0.2769 & 0.0403 & 1.7061 \\
\hline 257 & 6.5436 & 7.1711 & 1.4481 & 15.1628 \\
\hline 258 & 9.3086 & 10.4233 & 2.2400 & 21.9719 \\
\hline 259 & 5.6233 & 5.5683 & 0.6069 & 11.7986 \\
\hline 260 & 7.4894 & 6.2489 & 1.9636 & 15.7019 \\
\hline 261 & 6.2650 & 6.2508 & 0.4281 & 12.9439 \\
\hline 262 & 5.4553 & 3.4372 & 0.7403 & 9.6328 \\
\hline 263 & 6.5325 & 3.3422 & 1.0381 & 10.9128 \\
\hline 264 & 2.8644 & 1.8372 & 0.0017 & 4.7033 \\
\hline 265 & 5.0219 & 2.5186 & 0.4931 & 8.0336 \\
\hline 266 & 3.3508 & 4.8842 & 0.3308 & 8.5658 \\
\hline 267 & 6.8775 & 3.9681 & 0.9269 & 11.7725 \\
\hline 268 & 4.0586 & 3.6394 & 0.4808 & 8.1789 \\
\hline 269 & 6.0308 & 4.3831 & 0.1931 & 10.6069 \\
\hline
\end{tabular}




\begin{tabular}{|c|c|c|c|c|}
\hline $\begin{array}{l}\text { Day } \\
\text { Index }\end{array}$ & $\begin{array}{l}\text { Driving Time } \\
\text { [hr] }\end{array}$ & $\begin{array}{l}\text { Idle Time } \\
\text { [hr] }\end{array}$ & $\begin{array}{l}\text { PTO Time } \\
\text { [hr] }\end{array}$ & $\begin{array}{l}\text { Total } \\
\text { [hr] }\end{array}$ \\
\hline 270 & 7.8050 & 4.8008 & 2.5244 & 15.1303 \\
\hline 271 & 5.5047 & 2.4025 & 0.3583 & 8.2656 \\
\hline 272 & 4.9281 & 3.0572 & 0.5606 & 8.5458 \\
\hline 273 & 1.4692 & 1.0333 & 0.0219 & 2.5244 \\
\hline 274 & 2.4342 & 1.5672 & 0.0233 & 4.0247 \\
\hline 275 & 4.1019 & 3.9972 & 2.0678 & 10.1669 \\
\hline 276 & 2.6706 & 2.5200 & 0.2050 & 5.3956 \\
\hline 277 & 4.6944 & 5.1997 & 1.3067 & 11.2008 \\
\hline 278 & 1.9956 & 1.7858 & 0.5536 & 4.3350 \\
\hline 279 & 2.9594 & 3.1997 & 0.7050 & 6.8642 \\
\hline 280 & 2.1136 & 1.6753 & 0.2306 & 4.0194 \\
\hline 281 & 2.8550 & 1.9019 & 0.2603 & 5.0172 \\
\hline 282 & 3.9000 & 3.5794 & 0.4636 & 7.9431 \\
\hline 283 & 1.1625 & 1.4361 & 0.1394 & 2.7381 \\
\hline 284 & 1.7456 & 1.0497 & 0.5617 & 3.3569 \\
\hline 285 & 1.7989 & 2.4092 & 0.0189 & 4.2269 \\
\hline 286 & 3.3367 & 2.9125 & 0.5525 & 6.8017 \\
\hline 287 & 1.9892 & 2.4892 & 0.3667 & 4.8450 \\
\hline 288 & 2.6222 & 1.7503 & 0.0703 & 4.4428 \\
\hline 289 & 3.6078 & 2.7450 & 0.6353 & 6.9881 \\
\hline 290 & 2.0044 & 1.0211 & 0.0311 & 3.0567 \\
\hline 291 & 2.4158 & 0.9047 & 0.2631 & 3.5836 \\
\hline 292 & 2.1356 & 2.3433 & 0.5028 & 4.9817 \\
\hline 293 & 2.2231 & 1.5903 & 0.5711 & 4.3844 \\
\hline 294 & 2.8644 & 3.1075 & 0.4919 & 6.4639 \\
\hline 295 & 0.2108 & 0.0633 & 0.0000 & 0.2742 \\
\hline 296 & 1.9014 & 1.8403 & 1.0394 & 4.7811 \\
\hline 297 & 2.9031 & 4.4192 & 0.6978 & 8.0200 \\
\hline 298 & 1.9625 & 3.3217 & 0.0542 & 5.3383 \\
\hline 299 & 2.0869 & 2.8408 & 1.1297 & 6.0575 \\
\hline 300 & 1.6258 & 1.6569 & 0.0347 & 3.3175 \\
\hline 301 & 4.5122 & 3.8894 & 0.6261 & 9.0278 \\
\hline 302 & 4.1278 & 1.6464 & 0.0475 & 5.8217 \\
\hline 303 & 6.2114 & 4.2344 & 0.3983 & 10.8442 \\
\hline
\end{tabular}




\begin{tabular}{|c|c|c|c|c|}
\hline $\begin{array}{l}\text { Day } \\
\text { Index }\end{array}$ & $\begin{array}{l}\text { Driving Time } \\
\text { [hr] }\end{array}$ & $\begin{array}{l}\text { Idle Time } \\
\text { [hr] }\end{array}$ & $\begin{array}{l}\text { PTO Time } \\
\text { [hr] }\end{array}$ & $\begin{array}{l}\text { Total } \\
\text { [hr] }\end{array}$ \\
\hline 304 & 2.6103 & 2.0922 & 0.0267 & 4.7292 \\
\hline 305 & 0.4117 & 0.1172 & 0.0283 & 0.5572 \\
\hline 306 & 3.4714 & 2.1828 & 0.8103 & 6.4644 \\
\hline 307 & 1.5125 & 0.6031 & 0.0250 & 2.1406 \\
\hline 308 & 5.5181 & 4.2489 & 2.6778 & 12.4447 \\
\hline 309 & 6.0225 & 2.0228 & 2.0078 & 10.0531 \\
\hline 310 & 4.0847 & 1.5842 & 0.0175 & 5.6864 \\
\hline 311 & 1.4900 & 0.2317 & 0.0156 & 1.7372 \\
\hline 312 & 3.5197 & 3.0561 & 0.8253 & 7.4011 \\
\hline 313 & 6.0636 & 4.5928 & 0.7078 & 11.3642 \\
\hline 314 & 2.2550 & 0.2897 & 0.0028 & 2.5475 \\
\hline 315 & 6.0408 & 0.2864 & 0.0072 & 6.3344 \\
\hline 316 & 3.1842 & 1.1014 & 0.7919 & 5.0775 \\
\hline 317 & 4.3528 & 1.1797 & 0.3344 & 5.8669 \\
\hline 318 & 4.8617 & 1.7694 & 0.0133 & 6.6444 \\
\hline 319 & 7.8217 & 6.0928 & 3.4814 & 17.3958 \\
\hline 320 & 3.0725 & 2.1761 & 2.1494 & 7.3981 \\
\hline 321 & 9.8300 & 2.0989 & 0.5414 & 12.4703 \\
\hline 322 & 3.2608 & 1.2592 & 0.2639 & 4.7839 \\
\hline 323 & 4.6886 & 1.1361 & 0.0192 & 5.8439 \\
\hline 324 & 4.9703 & 1.6731 & 1.4683 & 8.1117 \\
\hline 325 & 7.0594 & 1.6897 & 1.6003 & 10.3494 \\
\hline 326 & 2.9514 & 0.7147 & 0.1522 & 3.8183 \\
\hline 327 & 2.7103 & 1.0303 & 0.3067 & 4.0472 \\
\hline 328 & 1.6869 & 3.1344 & 0.6544 & 5.4758 \\
\hline 329 & 1.2556 & 0.4586 & 1.2414 & 2.9556 \\
\hline 330 & 4.8722 & 2.1028 & 0.4786 & 7.4536 \\
\hline 331 & 4.9086 & 1.5739 & 1.2694 & 7.7519 \\
\hline 332 & 4.5067 & 1.2261 & 0.0139 & 5.7467 \\
\hline 333 & 0.3953 & 0.5817 & 0.0017 & 0.9786 \\
\hline 334 & 3.1694 & 0.9753 & 0.1719 & 4.3167 \\
\hline 335 & 1.9167 & 1.8158 & 0.6769 & 4.4094 \\
\hline 336 & 3.9525 & 2.1372 & 0.1442 & 6.2339 \\
\hline 337 & 1.9008 & 1.7986 & 0.9186 & 4.6181 \\
\hline
\end{tabular}




\begin{tabular}{|c|c|c|c|c|}
\hline $\begin{array}{l}\text { Day } \\
\text { Index }\end{array}$ & $\begin{array}{l}\text { Driving Time } \\
\text { [hr] }\end{array}$ & $\begin{array}{l}\text { Idle Time } \\
\text { [hr] }\end{array}$ & $\begin{array}{l}\text { PTO Time } \\
\text { [hr] }\end{array}$ & $\begin{array}{l}\text { Total } \\
\text { [hr] }\end{array}$ \\
\hline 338 & 3.0481 & 2.2728 & 0.0072 & 5.3281 \\
\hline 339 & 1.6353 & 2.6542 & 0.0097 & 4.2992 \\
\hline 340 & 3.2456 & 0.9861 & 0.1361 & 4.3678 \\
\hline 341 & 0.7089 & 0.2456 & 0.0200 & 0.9744 \\
\hline 342 & 0.4639 & 0.8036 & 0.6519 & 1.9194 \\
\hline 343 & 2.8606 & 2.4422 & 0.1978 & 5.5006 \\
\hline 344 & 8.9050 & 5.6353 & 2.7747 & 17.3150 \\
\hline 345 & 3.6622 & 2.8753 & 1.1186 & 7.6561 \\
\hline 346 & 7.0847 & 8.2553 & 0.9394 & 16.2794 \\
\hline 347 & 6.9672 & 4.0506 & 0.3053 & 11.3231 \\
\hline 348 & 4.2142 & 5.9808 & 0.2322 & 10.4272 \\
\hline 349 & 4.8081 & 2.5267 & 0.3025 & 7.6372 \\
\hline 350 & 7.5653 & 5.7017 & 2.0178 & 15.2847 \\
\hline 351 & 7.4767 & 7.3844 & 1.3667 & 16.2278 \\
\hline 352 & 4.0661 & 0.4033 & 0.0139 & 4.4833 \\
\hline 353 & 7.4681 & 3.4344 & 0.4144 & 11.3169 \\
\hline 354 & 7.0947 & 3.0636 & 0.8211 & 10.9794 \\
\hline 355 & 9.8769 & 7.0625 & 2.9864 & 19.9258 \\
\hline 356 & 1.3897 & 1.8247 & 0.0033 & 3.2178 \\
\hline 357 & 12.1842 & 4.8597 & 0.5228 & 17.5667 \\
\hline 358 & 7.6122 & 2.2075 & 0.7036 & 10.5233 \\
\hline 359 & 6.0989 & 1.9458 & 0.8850 & 8.9297 \\
\hline 360 & 12.6169 & 2.2933 & 1.6603 & 16.5706 \\
\hline 361 & 9.3286 & 7.1444 & 0.2644 & 16.7375 \\
\hline 362 & 4.2700 & 2.1867 & 1.0025 & 7.4592 \\
\hline 363 & 0.2819 & 0.0058 & 0.0017 & 0.2894 \\
\hline 364 & 4.0700 & 0.9175 & 0.3011 & 5.2886 \\
\hline 365 & 0.1872 & 0.2008 & 0.0086 & 0.3967 \\
\hline 366 & 2.5231 & 0.2000 & 0.0031 & 2.7261 \\
\hline 367 & 3.7222 & 0.9628 & 0.3186 & 5.0036 \\
\hline 368 & 4.7286 & 4.1953 & 0.3878 & 9.3117 \\
\hline 369 & 3.2975 & 2.9639 & 1.5211 & 7.7825 \\
\hline 370 & 6.5836 & 5.0047 & 3.8181 & 15.4064 \\
\hline 371 & 4.1822 & 4.0694 & 0.1256 & 8.3772 \\
\hline
\end{tabular}




\begin{tabular}{|c|c|c|c|c|}
\hline $\begin{array}{l}\text { Day } \\
\text { Index }\end{array}$ & $\begin{array}{l}\text { Driving Time } \\
\text { [hr] }\end{array}$ & $\begin{array}{l}\text { Idle Time } \\
\text { [hr] }\end{array}$ & $\begin{array}{l}\text { PTO Time } \\
\text { [hr] }\end{array}$ & $\begin{array}{l}\text { Total } \\
\text { [hr] }\end{array}$ \\
\hline 372 & 3.4583 & 1.4333 & 0.0264 & 4.9181 \\
\hline 373 & 3.0614 & 1.1072 & 0.5017 & 4.6703 \\
\hline 374 & 2.3072 & 1.1919 & 1.1889 & 4.6881 \\
\hline 375 & 3.9511 & 2.4142 & 0.3572 & 6.7225 \\
\hline 376 & 2.9631 & 1.6686 & 0.5467 & 5.1783 \\
\hline 377 & 5.5425 & 3.7286 & 1.8583 & 11.1294 \\
\hline 378 & 4.0081 & 4.1275 & 0.3978 & 8.5333 \\
\hline 379 & 1.0233 & 0.6858 & 0.3342 & 2.0433 \\
\hline 380 & 1.8783 & 1.5417 & 0.3989 & 3.8189 \\
\hline 381 & 3.9117 & 1.0758 & 0.4289 & 5.4164 \\
\hline 382 & 5.3219 & 5.0803 & 1.9503 & 12.3525 \\
\hline 383 & 2.8706 & 6.0867 & 0.7569 & 9.7142 \\
\hline 384 & 1.1400 & 0.4242 & 0.0075 & 1.5717 \\
\hline 385 & 2.3122 & 0.7544 & 0.0369 & 3.1036 \\
\hline 386 & 1.3808 & 0.2444 & 0.0325 & 1.6578 \\
\hline 387 & 2.4667 & 2.9572 & 0.0525 & 5.4764 \\
\hline 388 & 4.4322 & 2.4975 & 0.2717 & 7.2014 \\
\hline 389 & 5.0328 & 2.5358 & 1.5608 & 9.1294 \\
\hline 390 & 3.5139 & 4.0114 & 0.5897 & 8.1150 \\
\hline 391 & 2.2225 & 0.1878 & 0.0944 & 2.5047 \\
\hline 392 & 3.6014 & 5.0550 & 1.1411 & 9.7975 \\
\hline 393 & 5.2942 & 1.4722 & 0.7050 & 7.4714 \\
\hline 394 & 4.0961 & 1.1619 & 0.3300 & 5.5881 \\
\hline 395 & 2.7397 & 0.7136 & 0.5575 & 4.0108 \\
\hline 396 & 1.3869 & 0.2494 & 0.4217 & 2.0581 \\
\hline 397 & 1.7547 & 1.0722 & 0.6103 & 3.4372 \\
\hline 398 & 1.9394 & 0.6158 & 0.0506 & 2.6058 \\
\hline 399 & 4.0153 & 0.7911 & 0.3650 & 5.1714 \\
\hline 400 & 3.4750 & 1.9039 & 1.1628 & 6.5417 \\
\hline 401 & 3.6208 & 1.7625 & 1.7642 & 7.1475 \\
\hline 402 & 4.1539 & 1.4431 & 0.2658 & 5.8628 \\
\hline 403 & 2.0494 & 1.1703 & 0.4447 & 3.6644 \\
\hline 404 & 2.5197 & 1.6475 & 0.0406 & 4.2078 \\
\hline 405 & 1.5636 & 1.6369 & 0.3981 & 3.5986 \\
\hline
\end{tabular}




\begin{tabular}{|c|c|c|c|c|}
\hline $\begin{array}{l}\text { Day } \\
\text { Index }\end{array}$ & $\begin{array}{l}\text { Driving Time } \\
\text { [hr] }\end{array}$ & $\begin{array}{l}\text { Idle Time } \\
\text { [hr] }\end{array}$ & $\begin{array}{l}\text { PTO Time } \\
\text { [hr] }\end{array}$ & $\begin{array}{l}\text { Total } \\
\text { [hr] }\end{array}$ \\
\hline 406 & 1.1794 & 0.4150 & 1.9633 & 3.5578 \\
\hline 407 & 0.3758 & 2.0639 & 0.0000 & 2.4397 \\
\hline 408 & 1.4064 & 0.9689 & 4.5528 & 6.9281 \\
\hline 409 & 1.3631 & 0.4817 & 0.7653 & 2.6100 \\
\hline 410 & 1.1239 & 0.2778 & 0.3450 & 1.7467 \\
\hline 411 & 0.7711 & 0.4250 & 4.6039 & 5.8000 \\
\hline 412 & 0.2908 & 0.0439 & 2.0969 & 2.4317 \\
\hline 413 & 0.7217 & 4.1689 & 0.5294 & 5.4200 \\
\hline 414 & 1.0456 & 1.8711 & 2.3178 & 5.2344 \\
\hline 415 & 1.0703 & 3.5147 & 0.2919 & 4.8769 \\
\hline 416 & 0.7617 & 1.8306 & 0.6189 & 3.2111 \\
\hline 417 & 1.4733 & 3.9789 & 1.7839 & 7.2361 \\
\hline 418 & 1.2592 & 2.2692 & 1.8928 & 5.4211 \\
\hline 419 & 0.8144 & 0.9714 & 2.0636 & 3.8494 \\
\hline 420 & 1.0875 & 0.9936 & 0.9419 & 3.0231 \\
\hline 421 & 0.9308 & 0.8322 & 0.3625 & 2.1256 \\
\hline 422 & 0.8792 & 1.2744 & 0.4728 & 2.6264 \\
\hline 423 & 1.8917 & 0.7564 & 1.9936 & 4.6417 \\
\hline 424 & 1.8967 & 2.5536 & 2.1119 & 6.5622 \\
\hline 425 & 1.0969 & 1.5675 & 1.5969 & 4.2614 \\
\hline 426 & 0.6383 & 2.1197 & 0.9375 & 3.6956 \\
\hline 427 & 1.3581 & 2.2444 & 6.3200 & 9.9225 \\
\hline 428 & 2.4594 & 0.8033 & 6.1497 & 9.4125 \\
\hline 429 & 1.5306 & 0.7600 & 1.2239 & 3.5144 \\
\hline 430 & 1.4775 & 0.7867 & 1.7428 & 4.0069 \\
\hline 431 & 3.4492 & 1.0711 & 3.2950 & 7.8153 \\
\hline 432 & 1.6756 & 1.8531 & 5.8956 & 9.4242 \\
\hline 433 & 0.2764 & 0.0267 & 4.2656 & 4.5686 \\
\hline 434 & 1.3361 & 0.0797 & 0.5364 & 1.9522 \\
\hline 435 & 1.6856 & 0.0881 & 0.0000 & 1.7736 \\
\hline 436 & 0.9392 & 0.0989 & 0.1217 & 1.1597 \\
\hline 437 & 0.2558 & 3.3033 & 0.5375 & 4.0967 \\
\hline 438 & 1.8686 & 0.0547 & 0.0000 & 1.9233 \\
\hline 439 & 1.9828 & 1.0367 & 2.4097 & 5.4292 \\
\hline
\end{tabular}




\begin{tabular}{|c|c|c|c|c|}
\hline $\begin{array}{l}\text { Day } \\
\text { Index }\end{array}$ & $\begin{array}{l}\text { Driving Time } \\
\text { [hr] }\end{array}$ & $\begin{array}{l}\text { Idle Time } \\
\text { [hr] }\end{array}$ & $\begin{array}{l}\text { PTO Time } \\
\text { [hr] }\end{array}$ & $\begin{array}{l}\text { Total } \\
\text { [hr] }\end{array}$ \\
\hline 440 & 1.2464 & 0.7556 & 1.9253 & 3.9272 \\
\hline 441 & 0.7619 & 0.2569 & 0.0122 & 1.0311 \\
\hline 442 & 2.0489 & 1.4594 & 2.8097 & 6.3181 \\
\hline 443 & 0.6442 & 0.3311 & 1.6011 & 2.5764 \\
\hline 444 & 0.5033 & 1.4014 & 1.0442 & 2.9489 \\
\hline 445 & 0.6597 & 1.3347 & 2.3383 & 4.3328 \\
\hline 446 & 0.3219 & 1.3403 & 0.4697 & 2.1319 \\
\hline 447 & 1.2736 & 6.4172 & 0.0000 & 7.6908 \\
\hline 448 & 0.9950 & 0.3289 & 5.1939 & 6.5178 \\
\hline 449 & 1.2814 & 2.2206 & 3.8519 & 7.3539 \\
\hline 450 & 1.2117 & 0.0847 & 0.0000 & 1.2964 \\
\hline 451 & 1.1044 & 0.7883 & 1.1456 & 3.0383 \\
\hline 452 & 0.1436 & 0.2761 & 8.9414 & 9.3611 \\
\hline 453 & 1.0725 & 2.6953 & 0.0000 & 3.7678 \\
\hline 454 & 0.6356 & 0.2686 & 1.4783 & 2.3825 \\
\hline 455 & 0.1217 & 0.9092 & 7.6075 & 8.6383 \\
\hline 456 & 2.4019 & 0.2078 & 0.4756 & 3.0853 \\
\hline 457 & 1.2364 & 0.1706 & 3.7958 & 5.2028 \\
\hline 458 & 0.9706 & 0.3506 & 1.0911 & 2.4122 \\
\hline 459 & 0.8231 & 0.0156 & 0.0200 & 0.8586 \\
\hline 460 & 0.4483 & 1.3750 & 2.7539 & 4.5772 \\
\hline 461 & 1.2628 & 2.3500 & 1.8500 & 5.4628 \\
\hline 462 & 0.6825 & 1.0978 & 3.0269 & 4.8072 \\
\hline 463 & 2.1192 & 2.6131 & 0.1664 & 4.8986 \\
\hline 464 & 0.8042 & 1.7992 & 1.7317 & 4.3350 \\
\hline 465 & 2.6844 & 2.2622 & 4.1061 & 9.0528 \\
\hline 466 & 1.1858 & 1.0425 & 2.3050 & 4.5333 \\
\hline 467 & 0.1297 & 1.3619 & 2.9419 & 4.4336 \\
\hline 468 & 0.6011 & 1.9214 & 1.6133 & 4.1358 \\
\hline 469 & 0.1861 & 1.7822 & 3.0244 & 4.9928 \\
\hline 470 & 0.1581 & 1.3878 & 3.1200 & 4.6658 \\
\hline 471 & 1.2292 & 1.2133 & 2.3417 & 4.7842 \\
\hline 472 & 0.7258 & 0.6094 & 2.5189 & 3.8542 \\
\hline 473 & 0.9631 & 3.3197 & 1.2586 & 5.5414 \\
\hline
\end{tabular}




\begin{tabular}{|c|c|c|c|c|}
\hline $\begin{array}{l}\text { Day } \\
\text { Index }\end{array}$ & $\begin{array}{l}\text { Driving Time } \\
\text { [hr] }\end{array}$ & $\begin{array}{l}\text { Idle Time } \\
\text { [hr] }\end{array}$ & $\begin{array}{l}\text { PTO Time } \\
\text { [hr] }\end{array}$ & $\begin{array}{l}\text { Total } \\
\text { [hr] }\end{array}$ \\
\hline 474 & 0.4300 & 1.9017 & 2.7603 & 5.0919 \\
\hline 475 & 1.3506 & 2.7272 & 1.5131 & 5.5908 \\
\hline 476 & 1.5064 & 1.7681 & 3.0186 & 6.2931 \\
\hline 477 & 1.0242 & 2.1461 & 1.9536 & 5.1239 \\
\hline 478 & 2.0681 & 1.3692 & 0.9208 & 4.3581 \\
\hline 479 & 1.3728 & 0.8294 & 1.8686 & 4.0708 \\
\hline 480 & 1.9367 & 1.1428 & 2.4681 & 5.5475 \\
\hline 481 & 0.7911 & 0.2278 & 2.3675 & 3.3864 \\
\hline 482 & 0.2686 & 0.6686 & 2.7003 & 3.6375 \\
\hline 483 & 0.1678 & 2.6075 & 0.2789 & 3.0542 \\
\hline 484 & 1.2053 & 1.4233 & 3.9119 & 6.5406 \\
\hline 485 & 3.6200 & 1.8803 & 2.7072 & 8.2075 \\
\hline 486 & 0.5844 & 0.2542 & 3.8158 & 4.6544 \\
\hline 487 & 3.2817 & 1.3178 & 1.3281 & 5.9275 \\
\hline 488 & 0.4644 & 5.0475 & 0.7311 & 6.2431 \\
\hline 489 & 0.9228 & 1.9233 & 3.0950 & 5.9411 \\
\hline 490 & 1.5964 & 6.5856 & 0.4789 & 8.6608 \\
\hline 491 & 2.1275 & 6.8217 & 3.1717 & 12.1208 \\
\hline 492 & 0.2775 & 6.8639 & 2.7553 & 9.8967 \\
\hline 493 & 1.9128 & 8.2847 & 2.8675 & 13.0650 \\
\hline 494 & 2.4203 & 2.5542 & 2.1442 & 7.1186 \\
\hline 495 & 1.8353 & 2.7150 & 1.0781 & 5.6283 \\
\hline 496 & 1.4114 & 1.7972 & 1.8147 & 5.0233 \\
\hline 497 & 2.3889 & 1.8197 & 4.3572 & 8.5658 \\
\hline 498 & 1.5153 & 0.6558 & 3.2286 & 5.3997 \\
\hline 499 & 0.7414 & 1.2558 & 1.0947 & 3.0919 \\
\hline 500 & 2.2458 & 1.2042 & 2.3117 & 5.7617 \\
\hline 501 & 2.7017 & 5.4428 & 3.8267 & 11.9711 \\
\hline 502 & 2.4442 & 6.2642 & 1.7269 & 10.4353 \\
\hline 503 & 1.2122 & 1.6131 & 3.0914 & 5.9167 \\
\hline 504 & 1.6544 & 2.1092 & 3.1303 & 6.8939 \\
\hline 505 & 1.2631 & 0.6681 & 2.1108 & 4.0419 \\
\hline 506 & 1.3397 & 0.4911 & 1.9875 & 3.8183 \\
\hline 507 & 1.5817 & 1.3828 & 1.5092 & 4.4736 \\
\hline
\end{tabular}




\begin{tabular}{|c|c|c|c|c|}
\hline $\begin{array}{l}\text { Day } \\
\text { Index }\end{array}$ & $\begin{array}{l}\text { Driving Time } \\
\text { [hr] }\end{array}$ & $\begin{array}{l}\text { Idle Time } \\
\text { [hr] }\end{array}$ & $\begin{array}{l}\text { PTO Time } \\
\text { [hr] }\end{array}$ & $\begin{array}{l}\text { Total } \\
\text { [hr] }\end{array}$ \\
\hline 508 & 1.7833 & 0.1275 & 1.3689 & 3.2797 \\
\hline 509 & 1.2136 & 0.7422 & 1.3383 & 3.2942 \\
\hline 510 & 0.7906 & 0.1797 & 0.3803 & 1.3506 \\
\hline 511 & 1.7356 & 0.2336 & 0.2003 & 2.1694 \\
\hline 512 & 1.8733 & 0.1161 & 0.0000 & 1.9894 \\
\hline 513 & 2.1806 & 1.0797 & 1.1994 & 4.4597 \\
\hline 514 & 1.8400 & 0.5119 & 2.8008 & 5.1528 \\
\hline 515 & 0.9047 & 0.6961 & 4.9717 & 6.5725 \\
\hline 516 & 1.2539 & 1.2875 & 0.1900 & 2.7314 \\
\hline 517 & 1.9650 & 0.6444 & 1.1783 & 3.7878 \\
\hline 518 & 2.4664 & 0.3181 & 0.2489 & 3.0333 \\
\hline 519 & 2.0289 & 1.3817 & 0.2803 & 3.6908 \\
\hline 520 & 0.9183 & 0.5542 & 2.9353 & 4.4078 \\
\hline 521 & 1.0511 & 3.0211 & 0.0000 & 4.0722 \\
\hline 522 & 0.1425 & 0.0942 & 1.7278 & 1.9644 \\
\hline 523 & 1.0692 & 1.4106 & 1.9878 & 4.4675 \\
\hline 524 & 1.3897 & 1.0953 & 3.4833 & 5.9683 \\
\hline 525 & 1.4528 & 0.8939 & 0.2239 & 2.5706 \\
\hline 526 & 0.7881 & 0.6078 & 0.4353 & 1.8311 \\
\hline 527 & 4.2258 & 0.7111 & 3.5122 & 8.4492 \\
\hline 528 & 0.6011 & 0.0581 & 1.4144 & 2.0736 \\
\hline 529 & 0.5456 & 0.0000 & 0.0000 & 0.5456 \\
\hline 530 & 0.2342 & 0.0103 & 3.5617 & 3.8061 \\
\hline 531 & 4.0292 & 1.3825 & 6.3017 & 11.7133 \\
\hline 532 & 2.2042 & 0.0336 & 5.8836 & 8.1214 \\
\hline 533 & 0.5239 & 1.4186 & 6.4142 & 8.3567 \\
\hline 534 & 1.0644 & 1.1894 & 0.4061 & 2.6600 \\
\hline 535 & 1.4336 & 3.2889 & 1.6383 & 6.3608 \\
\hline 536 & 2.5636 & 2.6522 & 0.5592 & 5.7750 \\
\hline 537 & 0.4164 & 2.9172 & 2.0253 & 5.3589 \\
\hline 538 & 0.4864 & 1.5694 & 4.1219 & 6.1778 \\
\hline 539 & 1.1831 & 4.0964 & 5.8631 & 11.1425 \\
\hline 540 & 0.4442 & 0.8806 & 5.1342 & 6.4589 \\
\hline 541 & 0.5253 & 0.5767 & 3.0703 & 4.1722 \\
\hline
\end{tabular}




\begin{tabular}{|c|c|c|c|c|}
\hline $\begin{array}{l}\text { Day } \\
\text { Index }\end{array}$ & $\begin{array}{l}\text { Driving Time } \\
\text { [hr] }\end{array}$ & $\begin{array}{l}\text { Idle Time } \\
\text { [hr] }\end{array}$ & $\begin{array}{l}\text { PTO Time } \\
\text { [hr] }\end{array}$ & $\begin{array}{l}\text { Total } \\
\text { [hr] }\end{array}$ \\
\hline 542 & 0.3322 & 1.5478 & 4.1886 & 6.0686 \\
\hline 543 & 0.3911 & 3.1933 & 6.4025 & 9.9869 \\
\hline 544 & 0.1006 & 0.5194 & 9.6772 & 10.2972 \\
\hline 545 & 0.1181 & 0.7497 & 12.7925 & 13.6603 \\
\hline 546 & 0.9728 & 1.0272 & 5.9936 & 7.9936 \\
\hline 547 & 0.7881 & 0.3656 & 7.9997 & 9.1533 \\
\hline 548 & 0.5253 & 0.8867 & 7.7094 & 9.1214 \\
\hline 549 & 4.4417 & 5.4775 & 0.0103 & 9.9294 \\
\hline 550 & 0.2917 & 0.7156 & 3.4967 & 4.5039 \\
\hline 551 & 0.4786 & 0.5619 & 2.2969 & 3.3375 \\
\hline 552 & 0.9158 & 0.2478 & 3.5169 & 4.6806 \\
\hline 553 & 0.1928 & 0.7956 & 12.3700 & 13.3583 \\
\hline 554 & 0.1211 & 1.0644 & 7.1725 & 8.3581 \\
\hline 555 & 0.2333 & 1.6917 & 9.1042 & 11.0292 \\
\hline 556 & 0.2953 & 0.9153 & 11.2736 & 12.4842 \\
\hline 557 & 1.7267 & 1.5675 & 2.6517 & 5.9458 \\
\hline 558 & 0.2272 & 0.9211 & 11.6714 & 12.8197 \\
\hline 559 & 0.1111 & 0.5706 & 6.3094 & 6.9911 \\
\hline 560 & 0.1792 & 0.4997 & 11.7542 & 12.4331 \\
\hline 561 & 0.1303 & 0.3761 & 7.3728 & 7.8792 \\
\hline 562 & 0.2153 & 0.4611 & 8.6136 & 9.2900 \\
\hline 563 & 0.2675 & 0.3642 & 5.9475 & 6.5792 \\
\hline 564 & 0.1256 & 0.8067 & 3.4828 & 4.4150 \\
\hline 565 & 0.3350 & 0.7256 & 7.2503 & 8.3108 \\
\hline 566 & 0.1214 & 3.5164 & 1.0983 & 4.7361 \\
\hline 567 & 0.2936 & 0.6853 & 1.0797 & 2.0586 \\
\hline 568 & 0.7717 & 5.4506 & 1.9772 & 8.1994 \\
\hline 569 & 0.9242 & 4.7689 & 2.8286 & 8.5217 \\
\hline 570 & 0.8786 & 4.3292 & 2.7639 & 7.9717 \\
\hline 571 & 0.4031 & 4.0728 & 5.2008 & 9.6767 \\
\hline 572 & 0.8825 & 2.2133 & 1.4406 & 4.5364 \\
\hline 573 & 1.2056 & 2.6878 & 0.7425 & 4.6358 \\
\hline 574 & 0.9528 & 4.8717 & 6.2878 & 12.1122 \\
\hline 575 & 1.0856 & 3.1067 & 8.3767 & 12.5689 \\
\hline
\end{tabular}




\begin{tabular}{|c|c|c|c|c|}
\hline $\begin{array}{l}\text { Day } \\
\text { Index }\end{array}$ & $\begin{array}{l}\text { Driving Time } \\
\text { [hr] }\end{array}$ & $\begin{array}{l}\text { Idle Time } \\
\text { [hr] }\end{array}$ & $\begin{array}{l}\text { PTO Time } \\
\text { [hr] }\end{array}$ & $\begin{array}{l}\text { Total } \\
\text { [hr] }\end{array}$ \\
\hline 576 & 0.9133 & 2.5647 & 2.0561 & 5.5342 \\
\hline 577 & 0.7056 & 4.2772 & 2.0981 & 7.0808 \\
\hline 578 & 0.5375 & 5.8864 & 2.8753 & 9.2992 \\
\hline 579 & 0.2683 & 2.1808 & 4.9133 & 7.3625 \\
\hline 580 & 0.7508 & 2.6944 & 5.2533 & 8.6986 \\
\hline 581 & 0.6050 & 1.3231 & 5.9736 & 7.9017 \\
\hline 582 & 0.5256 & 2.9917 & 2.3489 & 5.8661 \\
\hline 583 & 0.5347 & 3.1681 & 2.8606 & 6.5633 \\
\hline 584 & 0.5542 & 2.0386 & 3.8578 & 6.4506 \\
\hline 585 & 0.5600 & 3.1356 & 4.0111 & 7.7067 \\
\hline 586 & 0.2547 & 1.4575 & 0.7914 & 2.5036 \\
\hline 587 & 0.5725 & 4.7253 & 3.2875 & 8.5853 \\
\hline 588 & 0.3433 & 2.8753 & 8.2911 & 11.5097 \\
\hline 589 & 0.5350 & 3.1761 & 6.5175 & 10.2286 \\
\hline 590 & 2.2431 & 0.9719 & 1.1153 & 4.3303 \\
\hline 591 & 0.7611 & 1.2828 & 5.1256 & 7.1694 \\
\hline 592 & 1.7864 & 4.0986 & 6.8961 & 12.7811 \\
\hline 593 & 0.9244 & 2.6886 & 2.9292 & 6.5422 \\
\hline 594 & 1.9375 & 0.9294 & 3.9878 & 6.8547 \\
\hline 595 & 2.6131 & 2.7769 & 2.2744 & 7.6644 \\
\hline 596 & 0.5744 & 1.8106 & 1.2933 & 3.6783 \\
\hline 597 & 0.4317 & 1.6083 & 0.5839 & 2.6239 \\
\hline 598 & 0.3206 & 1.3000 & 8.1789 & 9.7994 \\
\hline 599 & 1.2225 & 2.7161 & 1.6617 & 5.6003 \\
\hline 600 & 1.3108 & 0.7433 & 0.5364 & 2.5906 \\
\hline 601 & 1.0086 & 1.8756 & 3.6058 & 6.4900 \\
\hline 602 & 1.9664 & 5.2744 & 1.7286 & 8.9694 \\
\hline 603 & 2.0856 & 2.0717 & 0.3786 & 4.5358 \\
\hline 604 & 1.3736 & 1.6811 & 2.2044 & 5.2592 \\
\hline 605 & 0.5714 & 1.4558 & 1.7733 & 3.8006 \\
\hline 606 & 0.3456 & 1.4147 & 0.0000 & 1.7603 \\
\hline 607 & 2.1139 & 6.4119 & 0.0000 & 8.5258 \\
\hline 608 & 1.1919 & 3.4286 & 0.0000 & 4.6206 \\
\hline 609 & 1.1300 & 5.0411 & 0.0350 & 6.2061 \\
\hline
\end{tabular}




\begin{tabular}{|c|c|c|c|c|}
\hline $\begin{array}{l}\text { Day } \\
\text { Index }\end{array}$ & $\begin{array}{l}\text { Driving Time } \\
\text { [hr] }\end{array}$ & $\begin{array}{l}\text { Idle Time } \\
\text { [hr] }\end{array}$ & $\begin{array}{l}\text { PTO Time } \\
\text { [hr] }\end{array}$ & $\begin{array}{l}\text { Total } \\
\text { [hr] }\end{array}$ \\
\hline 610 & 2.8603 & 2.6778 & 0.0000 & 5.5381 \\
\hline 611 & 1.6144 & 1.4894 & 0.0000 & 3.1039 \\
\hline 612 & 1.0675 & 5.4408 & 0.0000 & 6.5083 \\
\hline 613 & 1.9364 & 1.2447 & 0.0000 & 3.1811 \\
\hline 614 & 1.1639 & 0.4297 & 0.0000 & 1.5936 \\
\hline 615 & 1.3803 & 1.7083 & 0.0000 & 3.0886 \\
\hline 616 & 1.3767 & 4.8850 & 0.0000 & 6.2617 \\
\hline 617 & 1.7300 & 2.7736 & 4.0981 & 8.6017 \\
\hline 618 & 1.8222 & 0.5242 & 3.8575 & 6.2039 \\
\hline 619 & 3.2964 & 3.2442 & 1.3997 & 7.9403 \\
\hline 620 & 0.5158 & 0.2047 & 0.0000 & 0.7206 \\
\hline 621 & 2.8189 & 1.7564 & 5.4294 & 10.0047 \\
\hline 622 & 1.7267 & 0.7450 & 1.6425 & 4.1142 \\
\hline 623 & 3.0864 & 3.3819 & 0.7728 & 7.2411 \\
\hline 624 & 3.8603 & 0.5028 & 0.0000 & 4.3631 \\
\hline 625 & 1.9158 & 1.9092 & 2.1286 & 5.9536 \\
\hline 626 & 1.0356 & 3.4350 & 5.9311 & 10.4017 \\
\hline 627 & 1.7917 & 3.1644 & 3.1653 & 8.1214 \\
\hline 628 & 0.8081 & 0.3806 & 2.0917 & 3.2803 \\
\hline 629 & 1.0333 & 1.4142 & 1.3919 & 3.8394 \\
\hline 630 & 0.2881 & 0.8097 & 3.8903 & 4.9881 \\
\hline 631 & 0.7986 & 0.9614 & 0.0000 & 1.7600 \\
\hline 632 & 0.7100 & 2.4589 & 0.5106 & 3.6794 \\
\hline 633 & 0.2786 & 0.5639 & 0.0000 & 0.8425 \\
\hline 634 & 2.7633 & 3.5314 & 1.8397 & 8.1344 \\
\hline 635 & 1.7103 & 1.8397 & 0.1528 & 3.7028 \\
\hline 636 & 0.9744 & 0.8497 & 0.2122 & 2.0364 \\
\hline 637 & 0.2847 & 2.9678 & 8.2917 & 11.5442 \\
\hline 638 & 0.3011 & 0.6408 & 1.3558 & 2.2978 \\
\hline 639 & 0.1392 & 0.0031 & 0.0000 & 0.1422 \\
\hline 640 & 0.2139 & 0.5231 & 3.2050 & 3.9419 \\
\hline 641 & 0.1597 & 1.8750 & 0.0486 & 2.0833 \\
\hline 642 & 0.5675 & 0.2239 & 1.0464 & 1.8378 \\
\hline 643 & 0.7531 & 1.9556 & 1.7378 & 4.4464 \\
\hline
\end{tabular}




\begin{tabular}{|c|c|c|c|c|}
\hline $\begin{array}{l}\text { Day } \\
\text { Index }\end{array}$ & $\begin{array}{l}\text { Driving Time } \\
\text { [hr] }\end{array}$ & $\begin{array}{l}\text { Idle Time } \\
\text { [hr] }\end{array}$ & $\begin{array}{l}\text { PTO Time } \\
\text { [hr] }\end{array}$ & $\begin{array}{l}\text { Total } \\
\text { [hr] }\end{array}$ \\
\hline 644 & 1.1378 & 1.9900 & 0.6394 & 3.7672 \\
\hline 645 & 1.4106 & 0.4289 & 1.4003 & 3.2397 \\
\hline 646 & 1.6433 & 2.0553 & 0.0000 & 3.6986 \\
\hline 647 & 1.1894 & 1.4186 & 3.7875 & 6.3956 \\
\hline 648 & 0.4522 & 0.6539 & 0.0000 & 1.1061 \\
\hline 649 & 1.5756 & 0.6003 & 3.3872 & 5.5631 \\
\hline 650 & 1.7539 & 0.3086 & 0.0000 & 2.0625 \\
\hline 651 & 1.5244 & 0.9633 & 0.0000 & 2.4878 \\
\hline 652 & 1.6150 & 0.8569 & 2.7911 & 5.2631 \\
\hline 653 & 0.9425 & 0.8300 & 3.0767 & 4.8492 \\
\hline 654 & 2.0564 & 0.4128 & 2.3364 & 4.8056 \\
\hline 655 & 2.5283 & 2.0003 & 5.9161 & 10.4447 \\
\hline 656 & 1.5175 & 0.4458 & 3.6878 & 5.6511 \\
\hline 657 & 1.7803 & 1.0633 & 0.0000 & 2.8436 \\
\hline 658 & 1.5808 & 2.0892 & 1.5392 & 5.2092 \\
\hline 659 & 1.6175 & 0.8744 & 0.9339 & 3.4258 \\
\hline 660 & 1.2069 & 1.3092 & 2.6283 & 5.1444 \\
\hline 661 & 1.4411 & 1.6058 & 2.4092 & 5.4561 \\
\hline 662 & 2.3564 & 3.0831 & 0.0183 & 5.4578 \\
\hline 663 & 1.3264 & 0.6064 & 2.6642 & 4.5969 \\
\hline 664 & 0.9233 & 0.5839 & 2.3042 & 3.8114 \\
\hline 665 & 1.5544 & 0.3233 & 2.1933 & 4.0711 \\
\hline 666 & 1.3781 & 0.7467 & 0.3158 & 2.4406 \\
\hline 667 & 0.4906 & 0.1406 & 8.2389 & 8.8700 \\
\hline 668 & 0.5664 & 0.0000 & 0.4494 & 1.0158 \\
\hline 669 & 1.1089 & 0.4956 & 4.4497 & 6.0542 \\
\hline 670 & 1.1336 & 1.0003 & 4.5892 & 6.7231 \\
\hline 671 & 0.8867 & 0.4689 & 3.9406 & 5.2961 \\
\hline 672 & 2.2394 & 9.2417 & 2.4808 & 13.9619 \\
\hline 673 & 2.0803 & 8.2942 & 0.8156 & 11.1900 \\
\hline 674 & 2.0336 & 3.0367 & 0.5889 & 5.6592 \\
\hline 675 & 1.4911 & 5.1411 & 5.1378 & 11.7700 \\
\hline 676 & 1.8003 & 3.1581 & 1.8692 & 6.8275 \\
\hline 677 & 3.4169 & 3.0142 & 0.8297 & 7.2608 \\
\hline
\end{tabular}




\begin{tabular}{|c|c|c|c|c|}
\hline $\begin{array}{l}\text { Day } \\
\text { Index }\end{array}$ & $\begin{array}{l}\text { Driving Time } \\
\text { [hr] }\end{array}$ & $\begin{array}{l}\text { Idle Time } \\
\text { [hr] }\end{array}$ & $\begin{array}{l}\text { PTO Time } \\
\text { [hr] }\end{array}$ & $\begin{array}{l}\text { Total } \\
\text { [hr] }\end{array}$ \\
\hline 678 & 1.6789 & 2.3789 & 7.0961 & 11.1539 \\
\hline 679 & 2.7633 & 4.5472 & 0.2050 & 7.5156 \\
\hline 680 & 0.5825 & 0.4458 & 2.2192 & 3.2475 \\
\hline 681 & 0.5050 & 3.0133 & 0.8958 & 4.4142 \\
\hline 682 & 0.5361 & 3.0378 & 1.3167 & 4.8906 \\
\hline 683 & 0.4764 & 3.0700 & 0.6044 & 4.1508 \\
\hline 684 & 0.3133 & 2.9506 & 0.7236 & 3.9875 \\
\hline 685 & 0.2650 & 6.2672 & 2.8161 & 9.3483 \\
\hline 686 & 1.2858 & 1.7931 & 5.2411 & 8.3200 \\
\hline 687 & 1.3347 & 1.9417 & 0.4944 & 3.7708 \\
\hline 688 & 1.1278 & 3.1525 & 3.4472 & 7.7275 \\
\hline 689 & 0.5300 & 1.8717 & 2.4600 & 4.8617 \\
\hline 690 & 1.1239 & 0.8500 & 1.6319 & 3.6058 \\
\hline 691 & 1.0600 & 1.4194 & 2.7744 & 5.2539 \\
\hline 692 & 2.4653 & 2.2181 & 0.2897 & 4.9731 \\
\hline 693 & 1.8536 & 3.8806 & 4.7833 & 10.5175 \\
\hline 694 & 1.2161 & 1.7922 & 0.2939 & 3.3022 \\
\hline 695 & 2.1850 & 0.8675 & 5.7631 & 8.8156 \\
\hline 696 & 2.0311 & 2.5033 & 2.5700 & 7.1044 \\
\hline 697 & 1.0844 & 0.4111 & 2.5642 & 4.0597 \\
\hline 698 & 0.8775 & 1.2086 & 1.2072 & 3.2933 \\
\hline 699 & 1.4681 & 0.1092 & 1.7200 & 3.2972 \\
\hline 700 & 0.4981 & 0.3608 & 0.0000 & 0.8589 \\
\hline 701 & 2.0508 & 0.1386 & 1.7372 & 3.9267 \\
\hline 702 & 0.4331 & 0.0864 & 0.0000 & 0.5194 \\
\hline 703 & 0.4772 & 0.1906 & 0.6506 & 1.3183 \\
\hline 704 & 0.7497 & 0.0078 & 0.0000 & 0.7575 \\
\hline 705 & 0.7631 & 0.0192 & 0.0000 & 0.7822 \\
\hline 706 & 0.6864 & 0.9292 & 2.8547 & 4.4703 \\
\hline 707 & 0.7050 & 0.3731 & 4.0622 & 5.1403 \\
\hline 708 & 0.6500 & 1.6478 & 0.0297 & 2.3275 \\
\hline 709 & 0.5128 & 2.3586 & 0.8231 & 3.6944 \\
\hline 710 & 3.5633 & 2.8047 & 1.2642 & 7.6322 \\
\hline 711 & 0.6281 & 1.2578 & 4.4183 & 6.3042 \\
\hline
\end{tabular}




\begin{tabular}{|c|c|c|c|c|}
\hline $\begin{array}{l}\text { Day } \\
\text { Index }\end{array}$ & $\begin{array}{l}\text { Driving Time } \\
\text { [hr] }\end{array}$ & $\begin{array}{l}\text { Idle Time } \\
\text { [hr] }\end{array}$ & $\begin{array}{l}\text { PTO Time } \\
\text { [hr] }\end{array}$ & $\begin{array}{l}\text { Total } \\
\text { [hr] }\end{array}$ \\
\hline 712 & 1.4853 & 1.8153 & 3.1444 & 6.4450 \\
\hline 713 & 0.4003 & 0.4836 & 2.3969 & 3.2808 \\
\hline 714 & 0.6214 & 1.6058 & 3.5133 & 5.7406 \\
\hline 715 & 0.3797 & 1.4819 & 0.5994 & 2.4611 \\
\hline 716 & 0.9594 & 2.2825 & 3.3986 & 6.6406 \\
\hline 717 & 0.8181 & 1.4114 & 1.7778 & 4.0072 \\
\hline 718 & 1.1853 & 0.2356 & 1.5294 & 2.9503 \\
\hline 719 & 1.0314 & 0.5131 & 2.6669 & 4.2114 \\
\hline 720 & 0.6683 & 0.9617 & 1.9169 & 3.5469 \\
\hline 721 & 2.6222 & 1.3389 & 1.0344 & 4.9956 \\
\hline 722 & 1.1247 & 0.3700 & 0.8281 & 2.3228 \\
\hline 723 & 0.3708 & 0.4814 & 1.6044 & 2.4567 \\
\hline 724 & 0.4200 & 2.7833 & 3.0519 & 6.2553 \\
\hline 725 & 0.2439 & 0.9478 & 2.1944 & 3.3861 \\
\hline 726 & 0.6989 & 1.3033 & 3.2286 & 5.2308 \\
\hline 727 & 0.7794 & 0.9942 & 0.5575 & 2.3311 \\
\hline 728 & 0.7756 & 2.0536 & 3.1431 & 5.9722 \\
\hline 729 & 0.5786 & 0.9575 & 1.6397 & 3.1758 \\
\hline 730 & 1.0486 & 0.2750 & 1.1789 & 2.5025 \\
\hline 731 & 2.6233 & 0.4481 & 2.2353 & 5.3067 \\
\hline 732 & 0.1717 & 0.2869 & 0.0000 & 0.4586 \\
\hline 733 & 1.0914 & 0.2772 & 0.6511 & 2.0197 \\
\hline 734 & 0.5975 & 1.9678 & 2.0492 & 4.6144 \\
\hline 735 & 1.0478 & 0.3183 & 1.4308 & 2.7969 \\
\hline 736 & 0.5500 & 0.4042 & 0.6669 & 1.6211 \\
\hline 737 & 2.3467 & 2.0200 & 1.0314 & 5.3981 \\
\hline 738 & 0.7883 & 0.1775 & 3.6986 & 4.6644 \\
\hline 739 & 0.6531 & 0.2119 & 0.9542 & 1.8192 \\
\hline 740 & 1.2156 & 0.8194 & 1.4689 & 3.5039 \\
\hline 741 & 0.9436 & 0.6069 & 1.2081 & 2.7586 \\
\hline 742 & 0.5869 & 0.8972 & 2.0500 & 3.5342 \\
\hline 743 & 1.1900 & 0.8558 & 0.0000 & 2.0458 \\
\hline 744 & 1.1008 & 3.3633 & 2.1911 & 6.6553 \\
\hline 745 & 1.0053 & 0.5489 & 1.9125 & 3.4667 \\
\hline
\end{tabular}




\begin{tabular}{|c|c|c|c|c|}
\hline $\begin{array}{l}\text { Day } \\
\text { Index }\end{array}$ & $\begin{array}{l}\text { Driving Time } \\
\text { [hr] }\end{array}$ & $\begin{array}{l}\text { Idle Time } \\
\text { [hr] }\end{array}$ & $\begin{array}{l}\text { PTO Time } \\
\text { [hr] }\end{array}$ & $\begin{array}{l}\text { Total } \\
\text { [hr] }\end{array}$ \\
\hline 746 & 0.8358 & 0.9400 & 0.6031 & 2.3789 \\
\hline 747 & 2.2361 & 0.8483 & 1.8775 & 4.9619 \\
\hline 748 & 1.3531 & 0.2039 & 5.8939 & 7.4508 \\
\hline 749 & 0.3558 & 0.1894 & 3.7683 & 4.3136 \\
\hline 750 & 0.2389 & 0.0000 & 0.1050 & 0.3439 \\
\hline 751 & 0.4356 & 0.0944 & 1.5064 & 2.0364 \\
\hline 752 & 0.5414 & 0.3725 & 2.8711 & 3.7850 \\
\hline 753 & 1.5119 & 0.0489 & 0.0000 & 1.5608 \\
\hline 754 & 0.5144 & 1.5717 & 2.6564 & 4.7425 \\
\hline 755 & 0.9567 & 0.3756 & 2.1636 & 3.4958 \\
\hline 756 & 1.8094 & 3.3444 & 2.0614 & 7.2153 \\
\hline 757 & 1.4778 & 0.3011 & 1.8481 & 3.6269 \\
\hline 758 & 0.5236 & 1.0028 & 1.8839 & 3.4103 \\
\hline 759 & 0.3303 & 0.1631 & 0.3678 & 0.8611 \\
\hline 760 & 0.1014 & 0.1731 & 1.6308 & 1.9053 \\
\hline 761 & 0.5017 & 4.4300 & 1.5028 & 6.4344 \\
\hline 762 & 0.3944 & 0.6728 & 2.6481 & 3.7153 \\
\hline 763 & 0.3847 & 0.1178 & 0.1142 & 0.6167 \\
\hline 764 & 1.1969 & 2.3700 & 4.3761 & 7.9431 \\
\hline 765 & 1.2231 & 6.1106 & 0.7894 & 8.1231 \\
\hline 766 & 0.6728 & 4.3528 & 1.0183 & 6.0439 \\
\hline 767 & 0.7319 & 4.9231 & 0.4867 & 6.1417 \\
\hline 768 & 0.2831 & 8.0639 & 0.3417 & 8.6886 \\
\hline 769 & 0.7089 & 0.0083 & 0.0000 & 0.7172 \\
\hline 770 & 0.7192 & 2.5197 & 1.7217 & 4.9606 \\
\hline 771 & 2.3367 & 2.4642 & 1.7361 & 6.5369 \\
\hline 772 & 1.5683 & 0.8819 & 3.2122 & 5.6625 \\
\hline 773 & 0.7758 & 0.0086 & 0.0000 & 0.7844 \\
\hline 774 & 0.6236 & 0.6869 & 1.8858 & 3.1964 \\
\hline 775 & 0.6992 & 0.2433 & 1.6442 & 2.5867 \\
\hline 776 & 1.5383 & 0.2586 & 2.7297 & 4.5267 \\
\hline 777 & 0.6183 & 3.3256 & 3.0097 & 6.9536 \\
\hline 778 & 0.9892 & 1.2369 & 3.9456 & 6.1717 \\
\hline 779 & 1.2533 & 0.3172 & 0.9039 & 2.4744 \\
\hline
\end{tabular}




\begin{tabular}{|c|c|c|c|c|}
\hline $\begin{array}{l}\text { Day } \\
\text { Index }\end{array}$ & $\begin{array}{l}\text { Driving Time } \\
\text { [hr] }\end{array}$ & $\begin{array}{l}\text { Idle Time } \\
\text { [hr] }\end{array}$ & $\begin{array}{l}\text { PTO Time } \\
\text { [hr] }\end{array}$ & $\begin{array}{l}\text { Total } \\
\text { [hr] }\end{array}$ \\
\hline 780 & 0.9258 & 1.5900 & 0.1183 & 2.6342 \\
\hline 781 & 2.2711 & 1.3483 & 3.4361 & 7.0556 \\
\hline 782 & 1.3931 & 0.3328 & 0.0000 & 1.7258 \\
\hline 783 & 2.4522 & 4.9269 & 0.2125 & 7.5917 \\
\hline 784 & 1.1083 & 1.3872 & 0.1567 & 2.6522 \\
\hline 785 & 0.6811 & 2.5903 & 5.7731 & 9.0444 \\
\hline 786 & 0.6903 & 0.1639 & 2.3967 & 3.2508 \\
\hline 787 & 2.6242 & 1.4750 & 1.7603 & 5.8594 \\
\hline 788 & 1.8761 & 0.2836 & 0.2319 & 2.3917 \\
\hline 789 & 0.7483 & 0.1253 & 2.5667 & 3.4403 \\
\hline 790 & 1.1703 & 0.7017 & 1.5122 & 3.3842 \\
\hline 791 & 1.4772 & 5.2764 & 1.3733 & 8.1269 \\
\hline 792 & 1.4158 & 0.5922 & 4.8411 & 6.8492 \\
\hline 793 & 3.2142 & 1.3864 & 1.5019 & 6.1025 \\
\hline 794 & 2.1064 & 0.4253 & 0.6744 & 3.2061 \\
\hline 795 & 1.0478 & 3.1794 & 1.1589 & 5.3861 \\
\hline 796 & 0.8675 & 1.4328 & 2.4619 & 4.7622 \\
\hline 797 & 0.3578 & 0.0972 & 0.2261 & 0.6811 \\
\hline 798 & 1.5847 & 1.0400 & 1.8875 & 4.5122 \\
\hline 799 & 0.7794 & 4.1994 & 5.8944 & 10.8733 \\
\hline 800 & 1.4553 & 0.5967 & 0.1439 & 2.1958 \\
\hline 801 & 1.5781 & 2.5264 & 2.1106 & 6.2150 \\
\hline 802 & 1.3519 & 1.1622 & 5.1775 & 7.6917 \\
\hline 803 & 1.1906 & 0.3200 & 4.2314 & 5.7419 \\
\hline 804 & 0.5011 & 0.4269 & 3.5031 & 4.4311 \\
\hline 805 & 0.2842 & 0.3289 & 2.3094 & 2.9225 \\
\hline 806 & 0.6131 & 0.6161 & 4.1189 & 5.3481 \\
\hline 807 & 0.6925 & 0.1397 & 2.1944 & 3.0267 \\
\hline 808 & 0.3367 & 1.4286 & 0.7583 & 2.5236 \\
\hline 809 & 0.4375 & 0.3789 & 4.9822 & 5.7986 \\
\hline 810 & 0.4031 & 2.5572 & 2.5028 & 5.4631 \\
\hline 811 & 0.1019 & 0.6553 & 3.7072 & 4.4644 \\
\hline 812 & 0.1219 & 0.0253 & 0.0925 & 0.2397 \\
\hline 813 & 0.2531 & 0.0042 & 0.0000 & 0.2572 \\
\hline
\end{tabular}




\begin{tabular}{|c|c|c|c|c|}
\hline $\begin{array}{l}\text { Day } \\
\text { Index }\end{array}$ & $\begin{array}{l}\text { Driving Time } \\
\text { [hr] }\end{array}$ & $\begin{array}{l}\text { Idle Time } \\
\text { [hr] }\end{array}$ & $\begin{array}{l}\text { PTO Time } \\
\text { [hr] }\end{array}$ & $\begin{array}{l}\text { Total } \\
\text { [hr] }\end{array}$ \\
\hline 814 & 0.2192 & 0.0303 & 0.0000 & 0.2494 \\
\hline 815 & 0.2000 & 0.1014 & 0.0439 & 0.3453 \\
\hline 816 & 0.2508 & 0.1578 & 4.7286 & 5.1372 \\
\hline 817 & 0.4492 & 0.7775 & 3.1308 & 4.3575 \\
\hline 818 & 0.4706 & 0.9728 & 4.9061 & 6.3494 \\
\hline 819 & 0.4428 & 0.0042 & 0.0000 & 0.4469 \\
\hline 820 & 0.1231 & 0.1269 & 0.0000 & 0.2500 \\
\hline 821 & 0.2014 & 0.0042 & 1.1756 & 1.3811 \\
\hline 822 & 0.1328 & 0.1200 & 0.0306 & 0.2833 \\
\hline 823 & 0.3264 & 0.5819 & 0.2917 & 1.2000 \\
\hline 824 & 0.1389 & 0.1167 & 0.0000 & 0.2556 \\
\hline 825 & 0.1425 & 0.0000 & 0.0000 & 0.1425 \\
\hline 826 & 0.2567 & 0.0000 & 0.2942 & 0.5508 \\
\hline 827 & 0.2125 & 0.0000 & 0.3239 & 0.5364 \\
\hline 828 & 0.1733 & 0.0042 & 0.0000 & 0.1775 \\
\hline 829 & 3.2617 & 0.5453 & 0.0000 & 3.8069 \\
\hline 830 & 0.1633 & 1.9128 & 1.0789 & 3.1550 \\
\hline 831 & 0.5236 & 0.5269 & 0.0139 & 1.0644 \\
\hline 832 & 1.0917 & 2.5342 & 0.9575 & 4.5833 \\
\hline 833 & 0.9369 & 2.6069 & 1.5100 & 5.0539 \\
\hline 834 & 2.3650 & 5.5175 & 3.3075 & 11.1900 \\
\hline 835 & 0.5008 & 2.1925 & 2.4719 & 5.1653 \\
\hline 836 & 0.6869 & 0.5047 & 4.7961 & 5.9878 \\
\hline 837 & 0.4425 & 1.7842 & 0.1139 & 2.3406 \\
\hline 838 & 0.3333 & 0.2794 & 3.4706 & 4.0833 \\
\hline 839 & 0.1556 & 0.0583 & 0.3472 & 0.5611 \\
\hline 840 & 0.5625 & 0.6967 & 2.8525 & 4.1117 \\
\hline 841 & 0.8072 & 0.5522 & 0.9289 & 2.2883 \\
\hline 842 & 2.2006 & 0.1203 & 1.5608 & 3.8817 \\
\hline 843 & 1.1269 & 0.8867 & 4.7903 & 6.8039 \\
\hline 844 & 0.4483 & 0.5292 & 7.6725 & 8.6500 \\
\hline 845 & 0.4983 & 1.0456 & 6.2483 & 7.7922 \\
\hline 846 & 1.8072 & 1.5156 & 9.6389 & 12.9617 \\
\hline 847 & 1.3086 & 0.5647 & 5.0611 & 6.9344 \\
\hline
\end{tabular}




\begin{tabular}{|c|c|c|c|c|}
\hline $\begin{array}{l}\text { Day } \\
\text { Index }\end{array}$ & $\begin{array}{l}\text { Driving Time } \\
\text { [hr] }\end{array}$ & $\begin{array}{l}\text { Idle Time } \\
\text { [hr] }\end{array}$ & $\begin{array}{l}\text { PTO Time } \\
\text { [hr] }\end{array}$ & $\begin{array}{l}\text { Total } \\
\text { [hr] }\end{array}$ \\
\hline 848 & 0.8758 & 0.2608 & 4.5517 & 5.6883 \\
\hline 849 & 1.0078 & 0.7994 & 5.0900 & 6.8972 \\
\hline 850 & 1.1978 & 1.2875 & 5.0569 & 7.5422 \\
\hline 851 & 0.9914 & 0.2842 & 4.4033 & 5.6789 \\
\hline 852 & 0.9164 & 1.0989 & 5.4486 & 7.4639 \\
\hline 853 & 0.1736 & 0.4625 & 7.4533 & 8.0894 \\
\hline 854 & 0.5300 & 0.2972 & 4.1736 & 5.0008 \\
\hline 855 & 0.2822 & 0.1831 & 1.4872 & 1.9525 \\
\hline 856 & 2.9742 & 0.7811 & 4.8778 & 8.6331 \\
\hline 857 & 0.4947 & 0.7519 & 6.8592 & 8.1058 \\
\hline 858 & 0.4975 & 0.6042 & 5.7864 & 6.8881 \\
\hline 859 & 0.4567 & 0.5067 & 7.5833 & 8.5467 \\
\hline 860 & 0.5922 & 0.1919 & 1.2686 & 2.0528 \\
\hline 861 & 0.9078 & 1.3958 & 2.4000 & 4.7036 \\
\hline 862 & 0.7911 & 0.1506 & 5.2292 & 6.1708 \\
\hline 863 & 1.6700 & 1.3394 & 2.7203 & 5.7297 \\
\hline 864 & 0.2667 & 0.4392 & 3.1022 & 3.8081 \\
\hline 865 & 0.2597 & 0.0233 & 2.2631 & 2.5461 \\
\hline 866 & 0.2133 & 0.1631 & 1.1256 & 1.5019 \\
\hline 867 & 0.4944 & 0.0228 & 1.6786 & 2.1958 \\
\hline 868 & 0.9108 & 0.0397 & 1.3564 & 2.3069 \\
\hline 869 & 0.7708 & 0.0928 & 5.2111 & 6.0747 \\
\hline 870 & 0.6664 & 0.0731 & 4.5314 & 5.2708 \\
\hline 871 & 0.6486 & 0.0111 & 6.1208 & 6.7806 \\
\hline 872 & 0.4292 & 0.0000 & 0.8914 & 1.3206 \\
\hline 873 & 0.3575 & 0.0000 & 0.0000 & 0.3575 \\
\hline 874 & 0.5539 & 0.2681 & 7.4694 & 8.2914 \\
\hline 875 & 0.8061 & 0.2158 & 5.7611 & 6.7831 \\
\hline 876 & 0.6731 & 0.3464 & 6.7503 & 7.7697 \\
\hline 877 & 0.9181 & 0.1222 & 1.5792 & 2.6194 \\
\hline 878 & 1.0214 & 0.1397 & 3.3419 & 4.5031 \\
\hline 879 & 1.0542 & 0.1814 & 1.4892 & 2.7247 \\
\hline 880 & 0.6014 & 0.2378 & 2.2603 & 3.0994 \\
\hline 881 & 0.6811 & 0.2181 & 0.0000 & 0.8992 \\
\hline
\end{tabular}




\begin{tabular}{|c|c|c|c|c|}
\hline $\begin{array}{l}\text { Day } \\
\text { Index }\end{array}$ & $\begin{array}{l}\text { Driving Time } \\
\text { [hr] }\end{array}$ & $\begin{array}{l}\text { Idle Time } \\
\text { [hr] }\end{array}$ & $\begin{array}{l}\text { PTO Time } \\
\text { [hr] }\end{array}$ & $\begin{array}{l}\text { Total } \\
\text { [hr] }\end{array}$ \\
\hline 882 & 0.4467 & 0.2056 & 0.0000 & 0.6522 \\
\hline 883 & 1.2097 & 5.1478 & 1.1933 & 7.5508 \\
\hline 884 & 1.1125 & 2.8578 & 1.0011 & 4.9714 \\
\hline 885 & 1.2675 & 1.9164 & 0.3292 & 3.5131 \\
\hline 886 & 1.5247 & 1.6744 & 0.0000 & 3.1992 \\
\hline 887 & 2.4500 & 0.6094 & 1.0936 & 4.1531 \\
\hline 888 & 1.4619 & 0.6867 & 0.0000 & 2.1486 \\
\hline 889 & 1.1922 & 0.5297 & 2.8539 & 4.5758 \\
\hline 890 & 1.7481 & 2.0517 & 2.1583 & 5.9581 \\
\hline 891 & 2.0028 & 0.8908 & 0.0164 & 2.9100 \\
\hline 892 & 1.2189 & 1.1239 & 0.9089 & 3.2517 \\
\hline 893 & 3.1303 & 5.0875 & 2.8572 & 11.0750 \\
\hline 894 & 1.1133 & 1.4072 & 3.4189 & 5.9394 \\
\hline 895 & 0.7544 & 0.8947 & 1.7767 & 3.4258 \\
\hline 896 & 0.7586 & 0.0950 & 4.9803 & 5.8339 \\
\hline 897 & 0.5225 & 0.7672 & 3.5897 & 4.8794 \\
\hline 898 & 0.8531 & 0.2742 & 1.4039 & 2.5311 \\
\hline 899 & 2.1839 & 2.4006 & 3.0231 & 7.6075 \\
\hline 900 & 1.0944 & 0.5631 & 5.5822 & 7.2397 \\
\hline 901 & 1.5425 & 0.7711 & 0.6886 & 3.0022 \\
\hline 902 & 1.3511 & 0.2350 & 1.1742 & 2.7603 \\
\hline 903 & 1.5475 & 1.0825 & 0.3581 & 2.9881 \\
\hline 904 & 1.8497 & 0.0950 & 3.6064 & 5.5511 \\
\hline 905 & 0.4344 & 3.6103 & 0.0000 & 4.0447 \\
\hline 906 & 0.5850 & 1.9194 & 1.8661 & 4.3706 \\
\hline 907 & 1.2667 & 0.0822 & 2.7906 & 4.1394 \\
\hline 908 & 1.4858 & 0.7508 & 0.3614 & 2.5981 \\
\hline 909 & 1.0161 & 0.3106 & 4.1706 & 5.4972 \\
\hline 910 & 1.3053 & 0.4803 & 0.5028 & 2.2883 \\
\hline 911 & 2.4428 & 0.2228 & 1.9781 & 4.6436 \\
\hline 912 & 2.3825 & 0.7422 & 4.0275 & 7.1522 \\
\hline 913 & 1.9211 & 1.1292 & 0.8717 & 3.9219 \\
\hline 914 & 0.4347 & 0.5592 & 0.1719 & 1.1658 \\
\hline 915 & 1.1031 & 0.0592 & 2.8997 & 4.0619 \\
\hline
\end{tabular}




\begin{tabular}{|c|c|c|c|c|}
\hline $\begin{array}{l}\text { Day } \\
\text { Index }\end{array}$ & $\begin{array}{l}\text { Driving Time } \\
\text { [hr] }\end{array}$ & $\begin{array}{l}\text { Idle Time } \\
\text { [hr] }\end{array}$ & $\begin{array}{l}\text { PTO Time } \\
\text { [hr] }\end{array}$ & $\begin{array}{l}\text { Total } \\
\text { [hr] }\end{array}$ \\
\hline 916 & 1.8317 & 0.7358 & 2.4344 & 5.0019 \\
\hline 917 & 1.7167 & 1.1089 & 2.5158 & 5.3414 \\
\hline 918 & 1.1611 & 0.0469 & 0.7925 & 2.0006 \\
\hline 919 & 0.8731 & 0.4461 & 1.0600 & 2.3792 \\
\hline 920 & 1.0969 & 0.4061 & 2.5303 & 4.0333 \\
\hline 921 & 0.6769 & 0.2550 & 2.2711 & 3.2031 \\
\hline 922 & 1.8194 & 0.2436 & 0.2950 & 2.3581 \\
\hline 923 & 1.3083 & 0.3064 & 0.3483 & 1.9631 \\
\hline 924 & 0.8164 & 0.1089 & 1.7525 & 2.6778 \\
\hline 925 & 1.4631 & 0.0347 & 0.5542 & 2.0519 \\
\hline 926 & 1.3381 & 0.0825 & 0.0000 & 1.4206 \\
\hline 927 & 1.4189 & 0.0569 & 5.4261 & 6.9019 \\
\hline 928 & 0.7331 & 3.7158 & 2.2047 & 6.6536 \\
\hline 929 & 0.3503 & 0.7244 & 0.0856 & 1.1603 \\
\hline 930 & 0.7317 & 0.7581 & 3.3703 & 4.8600 \\
\hline 931 & 0.6525 & 0.2503 & 1.3875 & 2.2903 \\
\hline 932 & 0.9825 & 0.2644 & 0.9994 & 2.2464 \\
\hline 933 & 3.9333 & 1.0672 & 1.9525 & 6.9531 \\
\hline 934 & 1.8575 & 0.1028 & 1.6947 & 3.6550 \\
\hline 935 & 2.2194 & 0.3117 & 3.6178 & 6.1489 \\
\hline 936 & 0.3811 & 0.0172 & 0.0000 & 0.3983 \\
\hline 937 & 0.7631 & 0.2192 & 0.5006 & 1.4828 \\
\hline 938 & 1.4303 & 0.1031 & 4.6289 & 6.1622 \\
\hline 939 & 0.3500 & 0.0453 & 0.0000 & 0.3953 \\
\hline 940 & 0.7272 & 0.1872 & 0.0000 & 0.9144 \\
\hline 941 & 2.3114 & 0.3844 & 2.3114 & 5.0072 \\
\hline 942 & 2.7400 & 0.1083 & 2.6092 & 5.4575 \\
\hline 943 & 1.4894 & 0.2772 & 0.5686 & 2.3353 \\
\hline 944 & 0.4939 & 0.0000 & 0.0000 & 0.4939 \\
\hline 945 & 1.4086 & 0.1547 & 3.1114 & 4.6747 \\
\hline 946 & 1.0686 & 0.2503 & 4.2042 & 5.5231 \\
\hline 947 & 3.2292 & 0.2431 & 0.5806 & 4.0528 \\
\hline 948 & 0.6697 & 0.0144 & 4.8611 & 5.5453 \\
\hline 949 & 0.7597 & 0.3797 & 0.0000 & 1.1394 \\
\hline
\end{tabular}




\begin{tabular}{|c|c|c|c|c|}
\hline $\begin{array}{l}\text { Day } \\
\text { Index }\end{array}$ & $\begin{array}{l}\text { Driving Time } \\
\text { [hr] }\end{array}$ & $\begin{array}{l}\text { Idle Time } \\
\text { [hr] }\end{array}$ & $\begin{array}{l}\text { PTO Time } \\
\text { [hr] }\end{array}$ & $\begin{array}{l}\text { Total } \\
\text { [hr] }\end{array}$ \\
\hline 950 & 0.7953 & 0.0450 & 0.0000 & 0.8403 \\
\hline 951 & 0.3025 & 0.8089 & 0.4961 & 1.6075 \\
\hline 952 & 2.4367 & 1.2667 & 0.4683 & 4.1717 \\
\hline 953 & 0.7389 & 0.3736 & 0.0000 & 1.1125 \\
\hline 954 & 0.6108 & 0.0739 & 1.4533 & 2.1381 \\
\hline 955 & 0.9100 & 0.5786 & 0.0772 & 1.5658 \\
\hline 956 & 0.4139 & 0.1169 & 0.0000 & 0.5308 \\
\hline 957 & 1.3086 & 0.4172 & 0.0117 & 1.7375 \\
\hline 958 & 0.6200 & 1.4408 & 1.7975 & 3.8583 \\
\hline 959 & 2.4092 & 0.2256 & 1.7272 & 4.3619 \\
\hline 960 & 0.2278 & 2.1225 & 2.0533 & 4.4036 \\
\hline 961 & 0.4528 & 0.4433 & 1.4908 & 2.3869 \\
\hline 962 & 0.5111 & 0.6725 & 3.3425 & 4.5261 \\
\hline 963 & 0.3586 & 0.0936 & 2.7656 & 3.2178 \\
\hline 964 & 0.6497 & 0.7125 & 1.3317 & 2.6939 \\
\hline 965 & 0.3225 & 0.4439 & 3.4756 & 4.2419 \\
\hline 966 & 0.4353 & 0.3983 & 1.2919 & 2.1256 \\
\hline 967 & 0.1550 & 0.4528 & 0.9878 & 1.5956 \\
\hline 968 & 0.2539 & 0.4142 & 1.4628 & 2.1308 \\
\hline 969 & 0.7233 & 1.1208 & 4.8061 & 6.6503 \\
\hline 970 & 0.4358 & 0.6503 & 0.2097 & 1.2958 \\
\hline 971 & 0.4428 & 0.2353 & 2.1589 & 2.8369 \\
\hline 972 & 0.1500 & 0.5892 & 4.1197 & 4.8589 \\
\hline 973 & 0.4147 & 1.0333 & 2.9189 & 4.3669 \\
\hline 974 & 0.1717 & 0.1083 & 2.0806 & 2.3606 \\
\hline 975 & 0.3983 & 0.3678 & 4.3308 & 5.0969 \\
\hline 976 & 0.5158 & 0.9133 & 0.2186 & 1.6478 \\
\hline 977 & 0.3825 & 0.9392 & 3.9194 & 5.2411 \\
\hline 978 & 0.3550 & 0.0528 & 0.0408 & 0.4486 \\
\hline 979 & 0.3203 & 0.5397 & 2.5694 & 3.4294 \\
\hline 980 & 0.1275 & 0.1906 & 3.5622 & 3.8803 \\
\hline 981 & 0.3992 & 0.3442 & 2.3067 & 3.0500 \\
\hline 982 & 0.1150 & 0.2203 & 2.5617 & 2.8969 \\
\hline 983 & 0.2011 & 0.3231 & 0.0000 & 0.5242 \\
\hline
\end{tabular}




\begin{tabular}{|c|c|c|c|c|}
\hline $\begin{array}{l}\text { Day } \\
\text { Index }\end{array}$ & $\begin{array}{l}\text { Driving Time } \\
\text { [hr] }\end{array}$ & $\begin{array}{l}\text { Idle Time } \\
\text { [hr] }\end{array}$ & $\begin{array}{l}\text { PTO Time } \\
\text { [hr] }\end{array}$ & $\begin{array}{l}\text { Total } \\
\text { [hr] }\end{array}$ \\
\hline 984 & 0.4078 & 2.3475 & 5.0747 & 7.8300 \\
\hline 985 & 0.3464 & 2.0117 & 2.0603 & 4.4183 \\
\hline 986 & 0.1286 & 0.5092 & 0.4494 & 1.0872 \\
\hline 987 & 0.1194 & 1.9119 & 7.9483 & 9.9797 \\
\hline 988 & 0.1031 & 0.2678 & 0.4022 & 0.7731 \\
\hline 989 & 0.2714 & 1.1742 & 0.7828 & 2.2283 \\
\hline 990 & 0.1747 & 1.6167 & 3.7739 & 5.5653 \\
\hline 991 & 0.6217 & 0.1797 & 3.1394 & 3.9408 \\
\hline 992 & 1.5225 & 0.4011 & 0.0000 & 1.9236 \\
\hline 993 & 0.8633 & 2.9617 & 2.0714 & 5.8964 \\
\hline 994 & 0.3739 & 0.0411 & 0.0000 & 0.4150 \\
\hline 995 & 1.5400 & 0.3133 & 2.7222 & 4.5756 \\
\hline 996 & 2.2422 & 0.8775 & 4.7431 & 7.8628 \\
\hline 997 & 0.8244 & 0.4075 & 1.9792 & 3.2111 \\
\hline 998 & 1.3800 & 0.1919 & 2.3411 & 3.9131 \\
\hline 999 & 0.3972 & 0.0528 & 2.7050 & 3.1550 \\
\hline 1000 & 1.4664 & 0.1078 & 2.4064 & 3.9806 \\
\hline 1001 & 1.8703 & 0.3686 & 4.4347 & 6.6736 \\
\hline 1002 & 0.6206 & 0.2078 & 0.0000 & 0.8283 \\
\hline 1003 & 0.5344 & 0.2983 & 3.9572 & 4.7900 \\
\hline 1004 & 0.5369 & 0.2097 & 0.4492 & 1.1958 \\
\hline 1005 & 0.1133 & 0.1572 & 0.1592 & 0.4297 \\
\hline 1006 & 1.8767 & 0.1031 & 0.4908 & 2.4706 \\
\hline 1007 & 0.9653 & 0.3669 & 0.4781 & 1.8103 \\
\hline 1008 & 1.3519 & 0.1436 & 0.0000 & 1.4956 \\
\hline 1009 & 1.3856 & 0.0942 & 0.0000 & 1.4797 \\
\hline 1010 & 0.5875 & 0.0000 & 0.0000 & 0.5875 \\
\hline 1011 & 0.3900 & 0.0000 & 0.9653 & 1.3553 \\
\hline 1012 & 1.8994 & 0.3153 & 0.0961 & 2.3108 \\
\hline 1013 & 0.4714 & 0.0000 & 0.0000 & 0.4714 \\
\hline 1014 & 0.2431 & 0.1900 & 5.9331 & 6.3661 \\
\hline 1015 & 0.1000 & 0.0133 & 0.0064 & 0.1197 \\
\hline 1016 & 0.2564 & 1.8475 & 0.3369 & 2.4408 \\
\hline 1017 & 0.1569 & 0.5322 & 2.7469 & 3.4361 \\
\hline
\end{tabular}




\begin{tabular}{|c|c|c|c|c|}
\hline $\begin{array}{l}\text { Day } \\
\text { Index }\end{array}$ & $\begin{array}{l}\text { Driving Time } \\
\text { [hr] }\end{array}$ & $\begin{array}{l}\text { Idle Time } \\
\text { [hr] }\end{array}$ & $\begin{array}{l}\text { PTO Time } \\
\text { [hr] }\end{array}$ & $\begin{array}{l}\text { Total } \\
\text { [hr] }\end{array}$ \\
\hline 1018 & 0.6361 & 0.4875 & 0.6053 & 1.7289 \\
\hline 1019 & 0.1519 & 0.0000 & 0.0000 & 0.1519 \\
\hline 1020 & 1.2186 & 0.2500 & 5.0122 & 6.4808 \\
\hline 1021 & 0.7783 & 0.0872 & 0.3319 & 1.1975 \\
\hline 1022 & 0.1736 & 0.4403 & 0.3572 & 0.9711 \\
\hline 1023 & 0.1817 & 0.0142 & 0.3589 & 0.5547 \\
\hline 1024 & 0.6344 & 1.7200 & 0.7556 & 3.1100 \\
\hline 1025 & 0.2889 & 0.3644 & 8.2917 & 8.9450 \\
\hline 1026 & 1.0592 & 0.3558 & 2.1003 & 3.5153 \\
\hline 1027 & 0.7069 & 0.0817 & 4.9656 & 5.7542 \\
\hline 1028 & 0.4342 & 0.0092 & 0.1625 & 0.6058 \\
\hline 1029 & 0.1400 & 0.0739 & 5.7772 & 5.9911 \\
\hline 1030 & 1.1858 & 0.0958 & 3.3219 & 4.6036 \\
\hline 1031 & 0.9783 & 0.2031 & 2.1128 & 3.2942 \\
\hline 1032 & 2.1792 & 0.3278 & 1.0678 & 3.5747 \\
\hline 1033 & 1.0947 & 0.2989 & 4.4286 & 5.8222 \\
\hline 1034 & 3.2394 & 0.3261 & 2.6794 & 6.2450 \\
\hline 1035 & 0.6478 & 0.2033 & 6.3172 & 7.1683 \\
\hline 1036 & 0.6936 & 0.9592 & 8.5175 & 10.1703 \\
\hline 1037 & 1.7711 & 0.6194 & 5.3000 & 7.6906 \\
\hline 1038 & 0.8097 & 0.3122 & 0.5789 & 1.7008 \\
\hline 1039 & 0.2203 & 0.1461 & 1.5203 & 1.8867 \\
\hline 1040 & 0.1839 & 0.0058 & 0.0000 & 0.1897 \\
\hline 1041 & 0.8097 & 0.1736 & 1.5753 & 2.5586 \\
\hline 1042 & 1.1497 & 0.2189 & 3.2836 & 4.6522 \\
\hline 1043 & 0.6683 & 0.2850 & 1.7122 & 2.6656 \\
\hline 1044 & 1.2300 & 0.0750 & 0.0000 & 1.3050 \\
\hline 1045 & 1.6183 & 0.0861 & 0.0000 & 1.7044 \\
\hline 1046 & 0.9269 & 0.2478 & 0.1522 & 1.3269 \\
\hline 1047 & 1.1097 & 0.1278 & 1.9258 & 3.1633 \\
\hline 1048 & 0.2675 & 0.4261 & 2.6264 & 3.3200 \\
\hline 1049 & 1.6925 & 0.9353 & 1.2517 & 3.8794 \\
\hline 1050 & 0.7225 & 1.0147 & 1.4861 & 3.2233 \\
\hline 1051 & 0.5153 & 1.6553 & 7.6339 & 9.8044 \\
\hline
\end{tabular}




\begin{tabular}{|c|c|c|c|c|}
\hline $\begin{array}{l}\text { Day } \\
\text { Index }\end{array}$ & $\begin{array}{l}\text { Driving Time } \\
\text { [hr] }\end{array}$ & $\begin{array}{l}\text { Idle Time } \\
\text { [hr] }\end{array}$ & $\begin{array}{l}\text { PTO Time } \\
\text { [hr] }\end{array}$ & $\begin{array}{l}\text { Total } \\
\text { [hr] }\end{array}$ \\
\hline 1052 & 0.8597 & 0.5197 & 0.0000 & 1.3794 \\
\hline 1053 & 1.3847 & 0.1989 & 1.3050 & 2.8886 \\
\hline 1054 & 0.6403 & 0.0247 & 0.0000 & 0.6650 \\
\hline 1055 & 2.4667 & 1.0386 & 3.4833 & 6.9886 \\
\hline 1056 & 0.4872 & 0.7892 & 1.1664 & 2.4428 \\
\hline 1057 & 0.1003 & 1.2444 & 1.7744 & 3.1192 \\
\hline 1058 & 1.4828 & 0.5806 & 2.6206 & 4.6839 \\
\hline 1059 & 1.1633 & 0.1758 & 1.3914 & 2.7306 \\
\hline 1060 & 0.5500 & 0.5253 & 1.7656 & 2.8408 \\
\hline 1061 & 1.4689 & 2.5200 & 3.9322 & 7.9211 \\
\hline 1062 & 2.0131 & 0.8483 & 0.0189 & 2.8803 \\
\hline 1063 & 1.3519 & 0.4817 & 0.0000 & 1.8336 \\
\hline 1064 & 1.1625 & 0.3214 & 3.0494 & 4.5333 \\
\hline 1065 & 1.2717 & 0.3419 & 1.4319 & 3.0456 \\
\hline 1066 & 1.1300 & 0.7525 & 2.1458 & 4.0283 \\
\hline 1067 & 1.2389 & 1.1931 & 0.1492 & 2.5811 \\
\hline 1068 & 1.4675 & 0.6319 & 1.6886 & 3.7881 \\
\hline 1069 & 1.8569 & 1.3808 & 1.9094 & 5.1472 \\
\hline 1070 & 0.6297 & 0.4878 & 2.0700 & 3.1875 \\
\hline 1071 & 2.2756 & 1.3236 & 0.1792 & 3.7783 \\
\hline 1072 & 0.7278 & 1.2231 & 3.5303 & 5.4811 \\
\hline 1073 & 0.5833 & 0.3003 & 2.2042 & 3.0878 \\
\hline 1074 & 1.3492 & 0.4603 & 1.0817 & 2.8911 \\
\hline 1075 & 1.6622 & 1.9214 & 0.0156 & 3.5992 \\
\hline 1076 & 1.7481 & 6.2806 & 0.0117 & 8.0403 \\
\hline 1077 & 1.4017 & 3.3089 & 0.8539 & 5.5644 \\
\hline 1078 & 0.1436 & 0.1092 & 0.0142 & 0.2669 \\
\hline 1079 & 0.9403 & 0.2681 & 2.0869 & 3.2953 \\
\hline 1080 & 0.5914 & 0.3917 & 1.6614 & 2.6444 \\
\hline 1081 & 0.1892 & 0.5311 & 0.2781 & 0.9983 \\
\hline 1082 & 0.7583 & 0.4853 & 4.3289 & 5.5725 \\
\hline 1083 & 0.5653 & 0.4017 & 4.7256 & 5.6925 \\
\hline 1084 & 0.8494 & 0.3578 & 4.9239 & 6.1311 \\
\hline 1085 & 1.0422 & 0.3861 & 1.6500 & 3.0783 \\
\hline
\end{tabular}




\begin{tabular}{|c|c|c|c|c|}
\hline $\begin{array}{l}\text { Day } \\
\text { Index }\end{array}$ & $\begin{array}{l}\text { Driving Time } \\
\text { [hr] }\end{array}$ & $\begin{array}{l}\text { Idle Time } \\
\text { [hr] }\end{array}$ & $\begin{array}{l}\text { PTO Time } \\
\text { [hr] }\end{array}$ & $\begin{array}{l}\text { Total } \\
\text { [hr] }\end{array}$ \\
\hline 1086 & 0.7381 & 0.2589 & 2.0403 & 3.0372 \\
\hline 1087 & 0.4222 & 0.0964 & 2.2942 & 2.8128 \\
\hline 1088 & 0.6219 & 1.9325 & 2.3731 & 4.9275 \\
\hline 1089 & 0.4167 & 2.1967 & 3.0750 & 5.6883 \\
\hline 1090 & 1.7272 & 2.7125 & 2.5639 & 7.0036 \\
\hline 1091 & 0.3956 & 2.6458 & 0.0947 & 3.1361 \\
\hline 1092 & 0.7706 & 0.9097 & 3.3653 & 5.0456 \\
\hline 1093 & 0.2578 & 1.2739 & 0.5208 & 2.0525 \\
\hline 1094 & 0.1050 & 0.0000 & 0.0000 & 0.1050 \\
\hline 1095 & 0.1414 & 0.0000 & 0.3019 & 0.4433 \\
\hline 1096 & 0.7711 & 0.0000 & 0.0000 & 0.7711 \\
\hline 1097 & 0.1061 & 0.0000 & 0.0000 & 0.1061 \\
\hline 1098 & 0.1436 & 0.0000 & 0.0000 & 0.1436 \\
\hline 1099 & 9.4569 & 0.0000 & 0.0000 & 9.4569 \\
\hline 1100 & 0.8153 & 0.0000 & 0.0000 & 0.8153 \\
\hline 1101 & 0.4978 & 0.0000 & 0.9561 & 1.4539 \\
\hline 1102 & 0.5181 & 0.0000 & 0.7883 & 1.3064 \\
\hline 1103 & 0.9031 & 0.0000 & 0.0000 & 0.9031 \\
\hline 1104 & 0.8539 & 0.0000 & 0.0000 & 0.8539 \\
\hline 1105 & 0.1411 & 0.0000 & 0.0000 & 0.1411 \\
\hline 1106 & 6.5244 & 0.0000 & 0.0000 & 6.5244 \\
\hline 1107 & 0.6156 & 0.0000 & 0.8106 & 1.4261 \\
\hline 1108 & 1.7456 & 0.0000 & 0.2553 & 2.0008 \\
\hline 1109 & 1.6500 & 0.0000 & 0.6861 & 2.3361 \\
\hline 1110 & 2.2094 & 0.0000 & 0.2878 & 2.4972 \\
\hline 1111 & 2.1217 & 0.0000 & 0.0000 & 2.1217 \\
\hline 1112 & 0.7158 & 0.0083 & 0.1419 & 0.8661 \\
\hline 1113 & 1.9975 & 3.0422 & 0.4361 & 5.4758 \\
\hline 1114 & 1.0989 & 3.5275 & 0.0000 & 4.6264 \\
\hline 1115 & 3.1625 & 5.2867 & 0.0000 & 8.4492 \\
\hline 1116 & 1.8536 & 5.2239 & 0.0900 & 7.1675 \\
\hline 1117 & 1.9836 & 4.6367 & 0.1625 & 6.7828 \\
\hline 1118 & 5.1472 & 5.2808 & 0.1142 & 10.5422 \\
\hline 1119 & 2.9883 & 7.3025 & 0.0000 & 10.2908 \\
\hline
\end{tabular}




\begin{tabular}{|c|c|c|c|c|}
\hline $\begin{array}{l}\text { Day } \\
\text { Index }\end{array}$ & $\begin{array}{l}\text { Driving Time } \\
\text { [hr] }\end{array}$ & $\begin{array}{l}\text { Idle Time } \\
\text { [hr] }\end{array}$ & $\begin{array}{l}\text { PTO Time } \\
\text { [hr] }\end{array}$ & $\begin{array}{l}\text { Total } \\
\text { [hr] }\end{array}$ \\
\hline 1120 & 4.3228 & 6.1861 & 0.0217 & 10.5306 \\
\hline 1121 & 3.7456 & 10.2661 & 0.7933 & 14.8050 \\
\hline 1122 & 2.9617 & 6.4386 & 0.0208 & 9.4211 \\
\hline 1123 & 0.7781 & 0.0000 & 0.0000 & 0.7781 \\
\hline 1124 & 0.2367 & 0.0000 & 0.2494 & 0.4861 \\
\hline 1125 & 1.0792 & 0.0000 & 0.0000 & 1.0792 \\
\hline 1126 & 0.3414 & 0.0000 & 0.4711 & 0.8125 \\
\hline 1127 & 0.1522 & 0.0000 & 0.4428 & 0.5950 \\
\hline 1128 & 1.1039 & 0.0000 & 0.1750 & 1.2789 \\
\hline 1129 & 0.5206 & 0.0544 & 0.0000 & 0.5750 \\
\hline 1130 & 1.1039 & 0.3819 & 0.0158 & 1.5017 \\
\hline 1131 & 0.4694 & 0.0686 & 0.0000 & 0.5381 \\
\hline 1132 & 0.8617 & 0.4094 & 0.5128 & 1.7839 \\
\hline 1133 & 2.1686 & 1.7447 & 0.6567 & 4.5700 \\
\hline 1134 & 0.7039 & 0.1200 & 0.0000 & 0.8239 \\
\hline 1135 & 1.3153 & 0.5175 & 0.0000 & 1.8328 \\
\hline 1136 & 0.4075 & 0.0078 & 0.0000 & 0.4153 \\
\hline 1137 & 1.5183 & 0.1794 & 0.0000 & 1.6978 \\
\hline 1138 & 1.3064 & 0.1233 & 0.4842 & 1.9139 \\
\hline 1139 & 0.9614 & 0.7719 & 0.5747 & 2.3081 \\
\hline 1140 & 0.7264 & 0.3114 & 1.0161 & 2.0539 \\
\hline 1141 & 2.1047 & 0.4486 & 0.0000 & 2.5533 \\
\hline 1142 & 1.1722 & 0.1756 & 0.0081 & 1.3558 \\
\hline 1143 & 1.1786 & 0.0514 & 0.0000 & 1.2300 \\
\hline 1144 & 1.5061 & 0.2217 & 0.0000 & 1.7278 \\
\hline 1145 & 0.6050 & 0.4536 & 0.6783 & 1.7369 \\
\hline 1146 & 1.3525 & 1.2317 & 0.0047 & 2.5889 \\
\hline 1147 & 0.1850 & 0.0092 & 0.0000 & 0.1942 \\
\hline 1148 & 0.2486 & 0.0175 & 0.0000 & 0.2661 \\
\hline 1149 & 2.5769 & 0.1672 & 0.1994 & 2.9436 \\
\hline 1150 & 1.7450 & 0.2628 & 0.4869 & 2.4947 \\
\hline 1151 & 0.6347 & 0.0297 & 0.6972 & 1.3617 \\
\hline 1152 & 1.2533 & 0.5497 & 0.0000 & 1.8031 \\
\hline 1153 & 2.4244 & 0.5414 & 3.7192 & 6.6850 \\
\hline
\end{tabular}




\begin{tabular}{|c|c|c|c|c|}
\hline $\begin{array}{l}\text { Day } \\
\text { Index }\end{array}$ & $\begin{array}{l}\text { Driving Time } \\
\text { [hr] }\end{array}$ & $\begin{array}{l}\text { Idle Time } \\
\text { [hr] }\end{array}$ & $\begin{array}{l}\text { PTO Time } \\
\text { [hr] }\end{array}$ & $\begin{array}{l}\text { Total } \\
\text { [hr] }\end{array}$ \\
\hline 1154 & 0.6992 & 0.0317 & 1.9289 & 2.6597 \\
\hline 1155 & 0.7514 & 0.1356 & 0.0031 & 0.8900 \\
\hline 1156 & 0.3800 & 0.1597 & 0.0531 & 0.5928 \\
\hline 1157 & 1.1111 & 1.5678 & 3.6392 & 6.3181 \\
\hline 1158 & 0.9419 & 0.1206 & 5.3208 & 6.3833 \\
\hline 1159 & 0.6275 & 0.5117 & 0.6050 & 1.7442 \\
\hline 1160 & 1.3800 & 0.0628 & 2.9383 & 4.3811 \\
\hline 1161 & 1.3067 & 1.1767 & 2.3122 & 4.7956 \\
\hline 1162 & 1.9544 & 0.3897 & 2.7803 & 5.1244 \\
\hline 1163 & 1.0761 & 0.1039 & 7.0761 & 8.2561 \\
\hline 1164 & 1.2528 & 0.3297 & 3.8964 & 5.4789 \\
\hline 1165 & 0.7106 & 0.2156 & 3.8903 & 4.8164 \\
\hline 1166 & 1.2839 & 2.4156 & 0.0000 & 3.6994 \\
\hline 1167 & 1.1939 & 0.4475 & 4.5625 & 6.2039 \\
\hline 1168 & 1.3897 & 0.7239 & 1.6136 & 3.7272 \\
\hline 1169 & 0.1858 & 0.6225 & 0.6083 & 1.4167 \\
\hline 1170 & 0.4747 & 0.0000 & 0.2144 & 0.6892 \\
\hline 1171 & 0.4944 & 0.0000 & 5.6081 & 6.1025 \\
\hline 1172 & 1.2644 & 0.0658 & 2.0467 & 3.3769 \\
\hline 1173 & 2.9417 & 0.0414 & 3.1128 & 6.0958 \\
\hline 1174 & 1.3506 & 0.1769 & 1.7986 & 3.3261 \\
\hline 1175 & 0.9861 & 0.0489 & 3.4903 & 4.5253 \\
\hline 1176 & 0.6964 & 0.0772 & 3.5944 & 4.3681 \\
\hline 1177 & 1.8675 & 0.6611 & 0.5447 & 3.0733 \\
\hline 1178 & 0.6439 & 0.0078 & 5.0586 & 5.7103 \\
\hline 1179 & 0.7242 & 0.4997 & 5.7050 & 6.9289 \\
\hline 1180 & 1.5878 & 0.0419 & 2.2611 & 3.8908 \\
\hline 1181 & 0.8347 & 0.0428 & 2.2481 & 3.1256 \\
\hline 1182 & 0.4794 & 0.0728 & 0.0000 & 0.5522 \\
\hline 1183 & 1.7206 & 0.0869 & 2.4964 & 4.3039 \\
\hline 1184 & 1.1433 & 0.5392 & 0.8250 & 2.5075 \\
\hline 1185 & 1.2889 & 0.0475 & 3.4681 & 4.8044 \\
\hline 1186 & 0.4589 & 0.5844 & 0.7942 & 1.8375 \\
\hline 1187 & 1.2850 & 0.3897 & 0.7267 & 2.4014 \\
\hline
\end{tabular}




\begin{tabular}{|c|c|c|c|c|}
\hline $\begin{array}{l}\text { Day } \\
\text { Index }\end{array}$ & $\begin{array}{l}\text { Driving Time } \\
\text { [hr] }\end{array}$ & $\begin{array}{l}\text { Idle Time } \\
\text { [hr] }\end{array}$ & $\begin{array}{l}\text { PTO Time } \\
\text { [hr] }\end{array}$ & $\begin{array}{l}\text { Total } \\
\text { [hr] }\end{array}$ \\
\hline 1188 & 1.2850 & 0.1425 & 0.7997 & 2.2272 \\
\hline 1189 & 3.4161 & 0.0711 & 0.0000 & 3.4872 \\
\hline 1190 & 0.2922 & 0.0000 & 0.0000 & 0.2922 \\
\hline 1191 & 0.2686 & 0.0000 & 3.7561 & 4.0247 \\
\hline 1192 & 0.9289 & 0.0000 & 0.1761 & 1.1050 \\
\hline 1193 & 0.1244 & 0.0000 & 0.0842 & 0.2086 \\
\hline 1194 & 0.5036 & 0.0000 & 0.0000 & 0.5036 \\
\hline 1195 & 0.2133 & 0.0000 & 1.2681 & 1.4814 \\
\hline 1196 & 0.1175 & 0.4594 & 0.0897 & 0.6667 \\
\hline 1197 & 0.2842 & 0.0339 & 0.0000 & 0.3181 \\
\hline 1198 & 6.3644 & 0.3361 & 0.0275 & 6.7281 \\
\hline 1199 & 0.1125 & 0.0767 & 0.0000 & 0.1892 \\
\hline 1200 & 1.1369 & 0.0000 & 0.0000 & 1.1369 \\
\hline 1201 & 0.2178 & 0.0000 & 7.3794 & 7.5972 \\
\hline 1202 & 0.7847 & 0.0000 & 0.0000 & 0.7847 \\
\hline 1203 & 0.9314 & 0.0000 & 0.1311 & 1.0625 \\
\hline 1204 & 1.1739 & 0.0000 & 0.0000 & 1.1739 \\
\hline 1205 & 0.3719 & 0.0000 & 0.0000 & 0.3719 \\
\hline 1206 & 0.3306 & 0.0000 & 0.0000 & 0.3306 \\
\hline 1207 & 0.3758 & 0.0000 & 0.6108 & 0.9867 \\
\hline 1208 & 0.4919 & 0.0000 & 1.4678 & 1.9597 \\
\hline 1209 & 0.3761 & 0.0000 & 0.0000 & 0.3761 \\
\hline 1210 & 1.1508 & 0.0000 & 0.2956 & 1.4464 \\
\hline 1211 & 4.1686 & 0.0000 & 0.0000 & 4.1686 \\
\hline 1212 & 0.9325 & 1.1017 & 2.0089 & 4.0431 \\
\hline 1213 & 0.6453 & 0.2392 & 1.9953 & 2.8797 \\
\hline 1214 & 0.8617 & 1.7644 & 1.2911 & 3.9172 \\
\hline 1215 & 0.7839 & 1.3875 & 1.8500 & 4.0214 \\
\hline 1216 & 1.1936 & 0.9353 & 2.2850 & 4.4139 \\
\hline 1217 & 0.7464 & 0.3931 & 5.9922 & 7.1317 \\
\hline 1218 & 1.0597 & 0.3978 & 5.8119 & 7.2694 \\
\hline 1219 & 1.1758 & 2.1056 & 3.6244 & 6.9058 \\
\hline 1220 & 0.7694 & 0.1000 & 6.5536 & 7.4231 \\
\hline 1221 & 1.0653 & 0.4550 & 6.4250 & 7.9453 \\
\hline
\end{tabular}




\begin{tabular}{|c|c|c|c|c|}
\hline $\begin{array}{l}\text { Day } \\
\text { Index }\end{array}$ & $\begin{array}{l}\text { Driving Time } \\
\text { [hr] }\end{array}$ & $\begin{array}{l}\text { Idle Time } \\
\text { [hr] }\end{array}$ & $\begin{array}{l}\text { PTO Time } \\
\text { [hr] }\end{array}$ & $\begin{array}{l}\text { Total } \\
\text { [hr] }\end{array}$ \\
\hline 1222 & 0.8697 & 1.3606 & 1.0639 & 3.2942 \\
\hline 1223 & 1.4103 & 1.5486 & 1.6167 & 4.5756 \\
\hline 1224 & 0.4453 & 0.0950 & 3.1453 & 3.6856 \\
\hline 1225 & 0.6167 & 0.5153 & 2.5500 & 3.6819 \\
\hline 1226 & 0.9978 & 1.8967 & 3.9994 & 6.8939 \\
\hline 1227 & 0.7239 & 0.0767 & 6.9892 & 7.7897 \\
\hline 1228 & 0.4503 & 0.0567 & 0.8381 & 1.3450 \\
\hline 1229 & 0.3808 & 0.0533 & 7.7292 & 8.1633 \\
\hline 1230 & 0.4378 & 0.0600 & 9.0594 & 9.5572 \\
\hline 1231 & 1.3733 & 0.8875 & 6.6419 & 8.9028 \\
\hline 1232 & 0.3831 & 0.0897 & 5.9733 & 6.4461 \\
\hline 1233 & 0.9061 & 0.0911 & 6.2422 & 7.2394 \\
\hline 1234 & 0.3536 & 1.0911 & 7.7353 & 9.1800 \\
\hline 1235 & 0.9256 & 0.5469 & 5.3972 & 6.8697 \\
\hline 1236 & 0.4378 & 0.5439 & 5.9917 & 6.9733 \\
\hline 1237 & 1.1822 & 0.5881 & 6.0867 & 7.8569 \\
\hline 1238 & 0.3575 & 0.6178 & 3.0581 & 4.0333 \\
\hline 1239 & 0.4606 & 0.5761 & 5.8233 & 6.8600 \\
\hline 1240 & 1.4814 & 2.3425 & 3.4861 & 7.3100 \\
\hline 1241 & 0.3917 & 0.1089 & 1.4958 & 1.9964 \\
\hline 1242 & 0.1586 & 0.3739 & 0.0000 & 0.5325 \\
\hline 1243 & 1.5053 & 0.7572 & 1.1528 & 3.4153 \\
\hline 1244 & 0.2653 & 2.3464 & 0.2119 & 2.8236 \\
\hline 1245 & 0.5000 & 0.5558 & 0.1450 & 1.2008 \\
\hline 1246 & 0.1242 & 0.7439 & 2.6100 & 3.4781 \\
\hline 1247 & 0.1969 & 0.3744 & 4.0233 & 4.5947 \\
\hline 1248 & 1.0572 & 0.3614 & 3.0414 & 4.4600 \\
\hline 1249 & 1.4103 & 0.5239 & 1.9192 & 3.8533 \\
\hline 1250 & 0.7047 & 0.5431 & 2.0078 & 3.2556 \\
\hline 1251 & 0.9669 & 0.6981 & 0.0853 & 1.7503 \\
\hline 1252 & 1.1333 & 0.2294 & 0.0000 & 1.3628 \\
\hline 1253 & 0.3806 & 0.2769 & 5.1283 & 5.7858 \\
\hline 1254 & 1.6739 & 0.5161 & 2.2372 & 4.4272 \\
\hline 1255 & 0.6508 & 0.2522 & 2.3575 & 3.2606 \\
\hline
\end{tabular}




\begin{tabular}{|c|c|c|c|c|}
\hline $\begin{array}{l}\text { Day } \\
\text { Index }\end{array}$ & $\begin{array}{l}\text { Driving Time } \\
\text { [hr] }\end{array}$ & $\begin{array}{l}\text { Idle Time } \\
\text { [hr] }\end{array}$ & $\begin{array}{l}\text { PTO Time } \\
\text { [hr] }\end{array}$ & $\begin{array}{l}\text { Total } \\
\text { [hr] }\end{array}$ \\
\hline 1256 & 0.5322 & 0.1919 & 0.1456 & 0.8697 \\
\hline 1257 & 0.1517 & 0.1344 & 0.0000 & 0.2861 \\
\hline 1258 & 12.2736 & 0.6972 & 0.0000 & 12.9708 \\
\hline 1259 & 0.9119 & 0.2356 & 0.2044 & 1.3519 \\
\hline 1260 & 0.2114 & 0.0033 & 0.8736 & 1.0883 \\
\hline 1261 & 0.7781 & 0.2783 & 1.5953 & 2.6517 \\
\hline 1262 & 0.2081 & 0.0503 & 0.0297 & 0.2881 \\
\hline 1263 & 0.2919 & 0.0706 & 0.0053 & 0.3678 \\
\hline 1264 & 0.1683 & 0.1131 & 4.8958 & 5.1772 \\
\hline 1265 & 0.5031 & 0.0603 & 0.8256 & 1.3889 \\
\hline 1266 & 1.3400 & 0.0983 & 2.7219 & 4.1603 \\
\hline 1267 & 1.3178 & 0.0489 & 2.7125 & 4.0792 \\
\hline 1268 & 1.2819 & 0.0408 & 1.3692 & 2.6919 \\
\hline 1269 & 1.3008 & 0.0122 & 1.3658 & 2.6789 \\
\hline 1270 & 1.2836 & 0.0247 & 2.6978 & 4.0061 \\
\hline 1271 & 1.2417 & 0.0364 & 2.6961 & 3.9742 \\
\hline 1272 & 1.3289 & 0.0203 & 2.7003 & 4.0494 \\
\hline 1273 & 1.3231 & 0.0186 & 2.7019 & 4.0436 \\
\hline 1274 & 1.2931 & 0.0364 & 2.7131 & 4.0425 \\
\hline 1275 & 1.2633 & 0.0172 & 2.3906 & 3.6711 \\
\hline 1276 & 1.2456 & 0.0217 & 2.7114 & 3.9786 \\
\hline 1277 & 1.2194 & 0.0206 & 2.3747 & 3.6147 \\
\hline 1278 & 0.6589 & 0.3381 & 0.8069 & 1.8039 \\
\hline 1279 & 2.6442 & 0.2869 & 2.2992 & 5.2303 \\
\hline 1280 & 2.7281 & 1.5589 & 0.0000 & 4.2869 \\
\hline 1281 & 1.8211 & 3.8094 & 0.1017 & 5.7322 \\
\hline 1282 & 1.8828 & 1.5828 & 1.7053 & 5.1708 \\
\hline 1283 & 1.8794 & 2.1272 & 1.8894 & 5.8961 \\
\hline 1284 & 1.6733 & 3.9586 & 0.3731 & 6.0050 \\
\hline 1285 & 1.3231 & 1.6578 & 3.2444 & 6.2253 \\
\hline 1286 & 1.6622 & 4.2486 & 0.3264 & 6.2372 \\
\hline 1287 & 0.8861 & 1.7989 & 3.1989 & 5.8839 \\
\hline 1288 & 0.7264 & 5.1356 & 0.3317 & 6.1936 \\
\hline 1289 & 1.3219 & 1.2647 & 0.4647 & 3.0514 \\
\hline
\end{tabular}




\begin{tabular}{|c|c|c|c|c|}
\hline $\begin{array}{l}\text { Day } \\
\text { Index }\end{array}$ & $\begin{array}{l}\text { Driving Time } \\
\text { [hr] }\end{array}$ & $\begin{array}{l}\text { Idle Time } \\
\text { [hr] }\end{array}$ & $\begin{array}{l}\text { PTO Time } \\
\text { [hr] }\end{array}$ & $\begin{array}{l}\text { Total } \\
\text { [hr] }\end{array}$ \\
\hline 1290 & 0.3842 & 1.3639 & 0.0081 & 1.7561 \\
\hline 1291 & 1.9386 & 0.3000 & 0.0000 & 2.2386 \\
\hline 1292 & 0.1367 & 1.2156 & 0.0000 & 1.3522 \\
\hline 1293 & 0.4353 & 0.3528 & 0.0000 & 0.7881 \\
\hline 1294 & 0.1817 & 0.0119 & 0.0000 & 0.1936 \\
\hline 1295 & 0.4250 & 0.1689 & 0.0131 & 0.6069 \\
\hline 1296 & 0.8492 & 3.9578 & 1.2883 & 6.0953 \\
\hline 1297 & 1.0719 & 0.0653 & 0.0000 & 1.1372 \\
\hline 1298 & 3.0222 & 1.6375 & 1.9278 & 6.5875 \\
\hline 1299 & 2.7964 & 1.6661 & 0.6950 & 5.1575 \\
\hline 1300 & 3.1686 & 2.6469 & 0.7253 & 6.5408 \\
\hline 1301 & 0.6392 & 2.1228 & 1.3639 & 4.1258 \\
\hline 1302 & 2.9036 & 4.1108 & 0.3633 & 7.3778 \\
\hline 1303 & 2.8567 & 3.2333 & 1.8947 & 7.9847 \\
\hline 1304 & 1.6100 & 3.4650 & 2.4228 & 7.4978 \\
\hline 1305 & 1.0025 & 0.4014 & 0.9297 & 2.3336 \\
\hline 1306 & 3.0569 & 2.8525 & 2.3317 & 8.2411 \\
\hline 1307 & 0.7675 & 7.0158 & 0.4489 & 8.2322 \\
\hline 1308 & 2.9072 & 0.5131 & 0.1325 & 3.5528 \\
\hline 1309 & 1.4886 & 0.3494 & 0.0000 & 1.8381 \\
\hline 1310 & 4.6322 & 3.3500 & 6.0600 & 14.0422 \\
\hline 1311 & 5.0317 & 0.9025 & 5.1122 & 11.0464 \\
\hline 1312 & 14.5106 & 0.1881 & 0.0214 & 14.7200 \\
\hline 1313 & 0.1550 & 0.2806 & 0.5158 & 0.9514 \\
\hline 1314 & 0.2814 & 1.8372 & 3.4308 & 5.5494 \\
\hline 1315 & 0.5272 & 0.0089 & 6.2911 & 6.8272 \\
\hline 1316 & 0.1889 & 0.4681 & 4.1000 & 4.7569 \\
\hline 1317 & 0.4797 & 0.0114 & 0.0000 & 0.4911 \\
\hline 1318 & 0.1828 & 0.2814 & 0.0000 & 0.4642 \\
\hline 1319 & 0.5939 & 0.4086 & 0.4747 & 1.4772 \\
\hline 1320 & 1.1028 & 0.9953 & 2.7406 & 4.8386 \\
\hline 1321 & 4.0656 & 0.6017 & 0.0000 & 4.6672 \\
\hline 1322 & 0.1144 & 0.2703 & 0.0958 & 0.4806 \\
\hline 1323 & 0.2833 & 0.0647 & 1.3792 & 1.7272 \\
\hline
\end{tabular}




\begin{tabular}{|c|c|c|c|c|}
\hline $\begin{array}{l}\text { Day } \\
\text { Index }\end{array}$ & $\begin{array}{l}\text { Driving Time } \\
\text { [hr] }\end{array}$ & $\begin{array}{l}\text { Idle Time } \\
\text { [hr] }\end{array}$ & $\begin{array}{l}\text { PTO Time } \\
\text { [hr] }\end{array}$ & $\begin{array}{l}\text { Total } \\
\text { [hr] }\end{array}$ \\
\hline 1324 & 0.1353 & 0.0900 & 1.6117 & 1.8369 \\
\hline 1325 & 0.6953 & 0.0192 & 0.0000 & 0.7144 \\
\hline 1326 & 0.1872 & 0.1708 & 2.7497 & 3.1078 \\
\hline 1327 & 0.1558 & 0.8581 & 0.1789 & 1.1928 \\
\hline 1328 & 0.1772 & 0.2117 & 4.1775 & 4.5664 \\
\hline 1329 & 7.0717 & 9.5364 & 0.0353 & 16.6433 \\
\hline 1330 & 12.6892 & 4.0158 & 0.0000 & 16.7050 \\
\hline 1331 & 5.8708 & 0.3572 & 0.0000 & 6.2281 \\
\hline 1332 & 0.4314 & 0.3392 & 1.0619 & 1.8325 \\
\hline 1333 & 0.2403 & 0.1542 & 0.0039 & 0.3983 \\
\hline 1334 & 0.2408 & 0.8428 & 1.6650 & 2.7486 \\
\hline 1335 & 0.1847 & 0.0939 & 1.2828 & 1.5614 \\
\hline 1336 & 0.1436 & 0.6731 & 0.0897 & 0.9064 \\
\hline 1337 & 0.3167 & 0.1242 & 0.7636 & 1.2044 \\
\hline 1338 & 5.0206 & 0.6728 & 2.3181 & 8.0114 \\
\hline 1339 & 12.9617 & 2.5194 & 0.0000 & 15.4811 \\
\hline 1340 & 7.5119 & 0.1519 & 0.0000 & 7.6639 \\
\hline 1341 & 0.3086 & 0.0633 & 0.0742 & 0.4461 \\
\hline 1342 & 0.2458 & 0.0558 & 0.2364 & 0.5381 \\
\hline 1343 & 0.1742 & 0.1989 & 0.0675 & 0.4406 \\
\hline 1344 & 2.3461 & 0.8764 & 0.7350 & 3.9575 \\
\hline 1345 & 1.9556 & 1.5483 & 0.0700 & 3.5739 \\
\hline 1346 & 2.5542 & 0.8906 & 0.4339 & 3.8786 \\
\hline 1347 & 3.0136 & 1.2875 & 0.7653 & 5.0664 \\
\hline 1348 & 0.2261 & 0.1472 & 0.1522 & 0.5256 \\
\hline 1349 & 0.9394 & 0.4661 & 0.3872 & 1.7928 \\
\hline 1350 & 3.5506 & 0.0967 & 0.3692 & 4.0164 \\
\hline 1351 & 2.0975 & 1.6186 & 0.0000 & 3.7161 \\
\hline 1352 & 2.1867 & 1.5747 & 0.0000 & 3.7614 \\
\hline 1353 & 0.3900 & 0.3853 & 0.2078 & 0.9831 \\
\hline 1354 & 1.0342 & 1.8131 & 1.1281 & 3.9753 \\
\hline 1355 & 1.5908 & 0.1158 & 0.3906 & 2.0972 \\
\hline 1356 & 1.3089 & 1.7350 & 0.3036 & 3.3475 \\
\hline 1357 & 0.5278 & 0.1317 & 0.8850 & 1.5444 \\
\hline
\end{tabular}




\begin{tabular}{|c|c|c|c|c|}
\hline $\begin{array}{l}\text { Day } \\
\text { Index }\end{array}$ & $\begin{array}{l}\text { Driving Time } \\
\text { [hr] }\end{array}$ & $\begin{array}{l}\text { Idle Time } \\
\text { [hr] }\end{array}$ & $\begin{array}{l}\text { PTO Time } \\
\text { [hr] }\end{array}$ & $\begin{array}{l}\text { Total } \\
\text { [hr] }\end{array}$ \\
\hline 1358 & 2.9925 & 0.7992 & 0.0408 & 3.8325 \\
\hline 1359 & 0.6817 & 0.8619 & 0.3425 & 1.8861 \\
\hline 1360 & 1.2314 & 0.3175 & 0.0000 & 1.5489 \\
\hline 1361 & 1.5250 & 0.2958 & 0.2158 & 2.0367 \\
\hline 1362 & 1.2156 & 2.1056 & 0.6328 & 3.9539 \\
\hline 1363 & 0.9992 & 0.6119 & 4.5958 & 6.2069 \\
\hline 1364 & 2.0339 & 1.1539 & 1.9994 & 5.1872 \\
\hline 1365 & 1.9294 & 0.1078 & 0.8544 & 2.8917 \\
\hline 1366 & 2.5342 & 1.2225 & 1.1536 & 4.9103 \\
\hline 1367 & 3.1436 & 1.5794 & 0.9256 & 5.6486 \\
\hline 1368 & 0.6233 & 0.6558 & 0.1711 & 1.4503 \\
\hline 1369 & 0.1108 & 0.0350 & 0.0000 & 0.1458 \\
\hline 1370 & 0.1306 & 0.2481 & 1.6164 & 1.9950 \\
\hline 1371 & 0.3147 & 0.2217 & 0.0000 & 0.5364 \\
\hline 1372 & 0.3092 & 0.0747 & 1.6278 & 2.0117 \\
\hline 1373 & 0.2894 & 0.1878 & 1.5311 & 2.0083 \\
\hline 1374 & 1.2700 & 1.1108 & 3.1247 & 5.5056 \\
\hline 1375 & 1.4367 & 0.9925 & 1.9567 & 4.3858 \\
\hline 1376 & 2.3694 & 1.4942 & 3.7458 & 7.6094 \\
\hline 1377 & 1.2783 & 0.0744 & 3.4917 & 4.8444 \\
\hline 1378 & 2.2603 & 2.4200 & 1.4658 & 6.1461 \\
\hline 1379 & 1.6069 & 3.3753 & 0.4364 & 5.4186 \\
\hline 1380 & 1.8944 & 1.4564 & 2.7658 & 6.1167 \\
\hline 1381 & 1.7061 & 2.0836 & 2.8714 & 6.6611 \\
\hline 1382 & 2.1428 & 1.1067 & 2.4303 & 5.6797 \\
\hline 1383 & 1.7744 & 2.7358 & 2.1019 & 6.6122 \\
\hline 1384 & 5.0394 & 2.9369 & 2.5944 & 10.5708 \\
\hline 1385 & 1.8544 & 1.8550 & 1.8900 & 5.5994 \\
\hline 1386 & 4.1933 & 1.4356 & 3.7400 & 9.3689 \\
\hline 1387 & 0.6253 & 0.4919 & 0.0239 & 1.1411 \\
\hline 1388 & 3.6450 & 0.8958 & 0.0111 & 4.5519 \\
\hline 1389 & 3.5167 & 0.7425 & 0.0000 & 4.2592 \\
\hline 1390 & 1.6439 & 1.5189 & 0.7639 & 3.9267 \\
\hline 1391 & 2.3778 & 1.3364 & 0.9067 & 4.6208 \\
\hline
\end{tabular}




\begin{tabular}{|c|c|c|c|c|}
\hline $\begin{array}{l}\text { Day } \\
\text { Index }\end{array}$ & $\begin{array}{l}\text { Driving Time } \\
\text { [hr] }\end{array}$ & $\begin{array}{l}\text { Idle Time } \\
\text { [hr] }\end{array}$ & $\begin{array}{l}\text { PTO Time } \\
\text { [hr] }\end{array}$ & $\begin{array}{l}\text { Total } \\
\text { [hr] }\end{array}$ \\
\hline 1392 & 1.7128 & 0.8725 & 0.6433 & 3.2286 \\
\hline 1393 & 0.2922 & 0.1472 & 0.0000 & 0.4394 \\
\hline 1394 & 2.0233 & 0.9908 & 0.7694 & 3.7836 \\
\hline 1395 & 1.6733 & 0.3336 & 3.7708 & 5.7778 \\
\hline 1396 & 1.6686 & 0.8772 & 1.7825 & 4.3283 \\
\hline 1397 & 1.8161 & 0.6975 & 2.5328 & 5.0464 \\
\hline 1398 & 2.0153 & 0.5503 & 1.9578 & 4.5233 \\
\hline 1399 & 1.5822 & 0.2358 & 2.6503 & 4.4683 \\
\hline 1400 & 2.0492 & 0.3994 & 2.3122 & 4.7608 \\
\hline 1401 & 0.9719 & 0.0000 & 0.8792 & 1.8511 \\
\hline 1402 & 0.2983 & 0.0000 & 0.0000 & 0.2983 \\
\hline 1403 & 1.1817 & 1.0358 & 3.0817 & 5.2992 \\
\hline 1404 & 10.0344 & 0.3189 & 1.2242 & 11.5775 \\
\hline 1405 & 0.9569 & 0.1664 & 0.0000 & 1.1233 \\
\hline 1406 & 0.4581 & 0.2481 & 0.0000 & 0.7061 \\
\hline 1407 & 1.6228 & 0.7356 & 0.0036 & 2.3619 \\
\hline 1408 & 0.4189 & 0.0381 & 0.0156 & 0.4725 \\
\hline 1409 & 0.3256 & 0.0381 & 0.8533 & 1.2169 \\
\hline 1410 & 1.7306 & 0.1569 & 1.0928 & 2.9803 \\
\hline 1411 & 0.3350 & 1.4381 & 3.8622 & 5.6353 \\
\hline 1412 & 0.5928 & 0.5122 & 0.0086 & 1.1136 \\
\hline 1413 & 0.3886 & 0.4075 & 0.0094 & 0.8056 \\
\hline 1414 & 0.5958 & 7.0397 & 0.2350 & 7.8706 \\
\hline 1415 & 0.5317 & 0.7644 & 2.9889 & 4.2850 \\
\hline 1416 & 1.9067 & 1.1464 & 3.0839 & 6.1369 \\
\hline 1417 & 0.5925 & 0.1003 & 1.1486 & 1.8414 \\
\hline 1418 & 0.3028 & 0.1625 & 0.0000 & 0.4653 \\
\hline 1419 & 0.2225 & 0.0161 & 0.0000 & 0.2386 \\
\hline 1420 & 2.7542 & 0.5139 & 1.2358 & 4.5039 \\
\hline 1421 & 2.0306 & 0.1236 & 0.5933 & 2.7475 \\
\hline 1422 & 2.2550 & 0.4531 & 1.3536 & 4.0617 \\
\hline 1423 & 1.4069 & 0.5378 & 0.7175 & 2.6622 \\
\hline 1424 & 1.7994 & 0.2600 & 0.2594 & 2.3189 \\
\hline 1425 & 1.8981 & 0.0683 & 0.2500 & 2.2164 \\
\hline
\end{tabular}




\begin{tabular}{|c|c|c|c|c|}
\hline $\begin{array}{l}\text { Day } \\
\text { Index }\end{array}$ & $\begin{array}{l}\text { Driving Time } \\
\text { [hr] }\end{array}$ & $\begin{array}{l}\text { Idle Time } \\
\text { [hr] }\end{array}$ & $\begin{array}{l}\text { PTO Time } \\
\text { [hr] }\end{array}$ & $\begin{array}{l}\text { Total } \\
\text { [hr] }\end{array}$ \\
\hline 1426 & 2.0692 & 0.1858 & 0.2775 & 2.5325 \\
\hline 1427 & 0.2619 & 0.3439 & 0.2686 & 0.8744 \\
\hline 1428 & 0.3317 & 0.0719 & 0.3647 & 0.7683 \\
\hline 1429 & 3.6528 & 0.4522 & 0.5494 & 4.6544 \\
\hline 1430 & 0.5308 & 0.0633 & 0.2939 & 0.8881 \\
\hline 1431 & 3.3944 & 0.5347 & 1.3636 & 5.2928 \\
\hline 1432 & 1.2061 & 0.1250 & 0.0000 & 1.3311 \\
\hline 1433 & 0.7944 & 0.1000 & 0.0000 & 0.8944 \\
\hline 1434 & 3.3972 & 0.4633 & 1.5886 & 5.4492 \\
\hline 1435 & 2.5342 & 0.4408 & 0.1025 & 3.0775 \\
\hline 1436 & 2.3569 & 0.6672 & 0.5456 & 3.5697 \\
\hline 1437 & 2.4136 & 0.2244 & 0.1458 & 2.7839 \\
\hline 1438 & 3.2094 & 0.4894 & 0.8217 & 4.5206 \\
\hline 1439 & 1.5167 & 0.2200 & 0.0000 & 1.7367 \\
\hline 1440 & 1.4769 & 0.3478 & 0.4458 & 2.2706 \\
\hline 1441 & 0.1181 & 0.4967 & 2.4261 & 3.0408 \\
\hline 1442 & 0.1456 & 4.6722 & 0.9444 & 5.7622 \\
\hline 1443 & 0.1061 & 0.0650 & 0.0000 & 0.1711 \\
\hline 1444 & 0.3206 & 0.2025 & 1.0542 & 1.5772 \\
\hline 1445 & 0.2606 & 0.1567 & 0.1686 & 0.5858 \\
\hline 1446 & 0.1614 & 0.0519 & 1.4669 & 1.6803 \\
\hline 1447 & 0.3786 & 0.5972 & 1.4278 & 2.4036 \\
\hline 1448 & 0.2722 & 0.6364 & 0.0039 & 0.9125 \\
\hline 1449 & 0.2433 & 0.9683 & 0.4494 & 1.6611 \\
\hline 1450 & 0.2906 & 0.0150 & 0.1339 & 0.4394 \\
\hline 1451 & 0.3658 & 0.0561 & 0.0000 & 0.4219 \\
\hline 1452 & 0.3803 & 0.0000 & 1.0850 & 1.4653 \\
\hline 1453 & 0.3219 & 0.0081 & 0.0000 & 0.3300 \\
\hline 1454 & 0.3458 & 0.0214 & 0.2469 & 0.6142 \\
\hline 1455 & 0.3286 & 0.0939 & 0.0000 & 0.4225 \\
\hline 1456 & 0.6158 & 0.0831 & 0.6647 & 1.3636 \\
\hline 1457 & 1.0983 & 1.2244 & 2.0125 & 4.3353 \\
\hline 1458 & 1.9225 & 2.3136 & 0.0422 & 4.2783 \\
\hline 1459 & 1.2661 & 0.3939 & 1.0153 & 2.6753 \\
\hline
\end{tabular}




\begin{tabular}{|c|c|c|c|c|}
\hline $\begin{array}{l}\text { Day } \\
\text { Index }\end{array}$ & $\begin{array}{l}\text { Driving Time } \\
\text { [hr] }\end{array}$ & $\begin{array}{l}\text { Idle Time } \\
\text { [hr] }\end{array}$ & $\begin{array}{l}\text { PTO Time } \\
\text { [hr] }\end{array}$ & $\begin{array}{l}\text { Total } \\
\text { [hr] }\end{array}$ \\
\hline 1460 & 0.8231 & 0.3872 & 12.2383 & 13.4486 \\
\hline 1461 & 0.5317 & 0.0381 & 1.1328 & 1.7025 \\
\hline 1462 & 0.3717 & 2.9569 & 0.3767 & 3.7053 \\
\hline 1463 & 3.6453 & 3.6997 & 1.4058 & 8.7508 \\
\hline 1464 & 1.8472 & 1.5017 & 3.9500 & 7.2989 \\
\hline 1465 & 1.2378 & 2.7814 & 1.3769 & 5.3961 \\
\hline 1466 & 1.8367 & 0.5153 & 0.4983 & 2.8503 \\
\hline 1467 & 1.7258 & 1.4917 & 0.5083 & 3.7258 \\
\hline 1468 & 1.3061 & 1.5119 & 2.3947 & 5.2128 \\
\hline 1469 & 1.7528 & 1.6044 & 2.2086 & 5.5658 \\
\hline 1470 & 0.9303 & 1.0478 & 4.4033 & 6.3814 \\
\hline 1471 & 0.8731 & 2.7950 & 2.1944 & 5.8625 \\
\hline 1472 & 0.3319 & 0.2453 & 0.2900 & 0.8672 \\
\hline 1473 & 0.7697 & 3.4575 & 0.2269 & 4.4542 \\
\hline 1474 & 1.4931 & 0.9961 & 3.8347 & 6.3239 \\
\hline 1475 & 1.5814 & 5.0536 & 0.0036 & 6.6386 \\
\hline 1476 & 1.7906 & 1.8222 & 0.0192 & 3.6319 \\
\hline 1477 & 0.6997 & 0.7031 & 1.5414 & 2.9442 \\
\hline 1478 & 0.4669 & 1.3244 & 3.9417 & 5.7331 \\
\hline 1479 & 1.2508 & 1.7053 & 0.8783 & 3.8344 \\
\hline 1480 & 1.0989 & 0.9136 & 0.5953 & 2.6078 \\
\hline 1481 & 1.0397 & 1.2186 & 2.7281 & 4.9864 \\
\hline 1482 & 1.9250 & 1.5025 & 0.7725 & 4.2000 \\
\hline 1483 & 0.2047 & 0.5317 & 0.0000 & 0.7364 \\
\hline 1484 & 0.7164 & 0.0000 & 0.0000 & 0.7164 \\
\hline 1485 & 3.5167 & 2.9925 & 1.2975 & 7.8067 \\
\hline 1486 & 0.4667 & 4.5492 & 0.1203 & 5.1361 \\
\hline 1487 & 0.5489 & 0.5489 & 0.1950 & 1.2928 \\
\hline 1488 & 2.5372 & 0.5039 & 0.0000 & 3.0411 \\
\hline 1489 & 1.2489 & 0.7350 & 0.5753 & 2.5592 \\
\hline 1490 & 2.2008 & 0.3314 & 0.6064 & 3.1386 \\
\hline 1491 & 0.6275 & 0.1067 & 0.0000 & 0.7342 \\
\hline 1492 & 0.2142 & 0.0000 & 0.0000 & 0.2142 \\
\hline 1493 & 0.6744 & 0.4331 & 5.6008 & 6.7083 \\
\hline
\end{tabular}




\begin{tabular}{|c|c|c|c|c|}
\hline $\begin{array}{l}\text { Day } \\
\text { Index }\end{array}$ & $\begin{array}{l}\text { Driving Time } \\
\text { [hr] }\end{array}$ & $\begin{array}{l}\text { Idle Time } \\
\text { [hr] }\end{array}$ & $\begin{array}{l}\text { PTO Time } \\
\text { [hr] }\end{array}$ & $\begin{array}{l}\text { Total } \\
\text { [hr] }\end{array}$ \\
\hline 1494 & 2.1192 & 0.3597 & 0.0000 & 2.4789 \\
\hline 1495 & 1.8606 & 3.7436 & 3.3525 & 8.9567 \\
\hline 1496 & 0.8383 & 1.1472 & 9.2997 & 11.2853 \\
\hline 1497 & 0.5417 & 0.0856 & 5.2533 & 5.8806 \\
\hline 1498 & 1.3681 & 0.1953 & 8.2364 & 9.7997 \\
\hline 1499 & 0.1075 & 0.5294 & 1.1842 & 1.8211 \\
\hline 1500 & 0.5606 & 1.1169 & 1.6978 & 3.3753 \\
\hline 1501 & 0.3481 & 0.6728 & 0.9953 & 2.0161 \\
\hline 1502 & 0.5308 & 3.8653 & 5.6181 & 10.0142 \\
\hline 1503 & 0.4272 & 2.3867 & 6.0806 & 8.8944 \\
\hline 1504 & 0.7722 & 2.0161 & 2.2986 & 5.0869 \\
\hline 1505 & 0.3331 & 3.2575 & 4.3281 & 7.9186 \\
\hline 1506 & 1.3328 & 0.5775 & 0.3133 & 2.2236 \\
\hline 1507 & 0.2017 & 1.5458 & 1.3547 & 3.1022 \\
\hline 1508 & 0.2386 & 1.4911 & 1.9864 & 3.7161 \\
\hline 1509 & 0.3425 & 0.4475 & 2.8675 & 3.6575 \\
\hline 1510 & 0.2464 & 2.0761 & 0.7128 & 3.0353 \\
\hline 1511 & 0.4089 & 2.8189 & 0.7003 & 3.9281 \\
\hline 1512 & 0.3525 & 0.8497 & 0.7214 & 1.9236 \\
\hline 1513 & 0.3531 & 1.4525 & 1.5708 & 3.3764 \\
\hline 1514 & 0.3642 & 2.9108 & 0.2703 & 3.5453 \\
\hline 1515 & 0.3703 & 1.3356 & 5.3317 & 7.0375 \\
\hline 1516 & 0.4094 & 1.6833 & 1.9539 & 4.0467 \\
\hline 1517 & 0.6569 & 2.5722 & 0.6036 & 3.8328 \\
\hline 1518 & 0.2456 & 3.1667 & 0.1717 & 3.5839 \\
\hline 1519 & 0.3017 & 0.7775 & 0.9378 & 2.0169 \\
\hline 1520 & 0.1400 & 0.2644 & 0.7458 & 1.1503 \\
\hline 1521 & 0.1219 & 2.9322 & 5.3936 & 8.4478 \\
\hline 1522 & 0.3731 & 1.5817 & 2.2953 & 4.2500 \\
\hline 1523 & 0.5394 & 0.5817 & 8.9675 & 10.0886 \\
\hline 1524 & 0.4986 & 1.8058 & 3.4264 & 5.7308 \\
\hline 1525 & 0.2511 & 0.3422 & 7.3506 & 7.9439 \\
\hline 1526 & 0.2231 & 0.7414 & 5.5528 & 6.5172 \\
\hline 1527 & 0.6286 & 1.2411 & 4.6439 & 6.5136 \\
\hline
\end{tabular}




\begin{tabular}{|c|c|c|c|c|}
\hline $\begin{array}{l}\text { Day } \\
\text { Index }\end{array}$ & $\begin{array}{l}\text { Driving Time } \\
\text { [hr] }\end{array}$ & $\begin{array}{l}\text { Idle Time } \\
\text { [hr] }\end{array}$ & $\begin{array}{l}\text { PTO Time } \\
\text { [hr] }\end{array}$ & $\begin{array}{l}\text { Total } \\
\text { [hr] }\end{array}$ \\
\hline 1528 & 1.1747 & 2.3950 & 3.6836 & 7.2533 \\
\hline 1529 & 1.0436 & 2.4747 & 2.1867 & 5.7050 \\
\hline 1530 & 0.5944 & 4.7300 & 4.9656 & 10.2900 \\
\hline 1531 & 0.9953 & 2.6047 & 4.2875 & 7.8875 \\
\hline 1532 & 0.2508 & 6.8842 & 0.1117 & 7.2467 \\
\hline 1533 & 0.2925 & 2.2678 & 3.5911 & 6.1514 \\
\hline 1534 & 0.8742 & 2.9289 & 8.5947 & 12.3978 \\
\hline 1535 & 0.6450 & 1.0681 & 4.4144 & 6.1275 \\
\hline 1536 & 1.3800 & 1.7250 & 5.5550 & 8.6600 \\
\hline 1537 & 0.6456 & 2.8297 & 2.6625 & 6.1378 \\
\hline 1538 & 0.2311 & 0.1222 & 0.0000 & 0.3533 \\
\hline 1539 & 1.1792 & 0.6000 & 5.0575 & 6.8367 \\
\hline 1540 & 0.7050 & 1.7386 & 7.9497 & 10.3933 \\
\hline 1541 & 0.3847 & 2.5028 & 3.7042 & 6.5917 \\
\hline 1542 & 0.3106 & 0.1239 & 1.7517 & 2.1861 \\
\hline 1543 & 0.3444 & 0.8933 & 3.5783 & 4.8161 \\
\hline 1544 & 0.7717 & 1.0839 & 5.3575 & 7.2131 \\
\hline 1545 & 0.9472 & 1.1575 & 9.0428 & 11.1475 \\
\hline 1546 & 0.7689 & 0.8497 & 6.0283 & 7.6469 \\
\hline 1547 & 0.4858 & 1.7411 & 9.0906 & 11.3175 \\
\hline 1548 & 0.5986 & 0.5194 & 8.9547 & 10.0728 \\
\hline 1549 & 0.3350 & 0.1628 & 1.5403 & 2.0381 \\
\hline 1550 & 0.3022 & 0.4067 & 6.4789 & 7.1878 \\
\hline 1551 & 0.8392 & 0.9908 & 2.9811 & 4.8111 \\
\hline 1552 & 0.7458 & 0.5533 & 9.3508 & 10.6500 \\
\hline 1553 & 0.8606 & 0.9639 & 4.0769 & 5.9014 \\
\hline 1554 & 1.5722 & 1.7569 & 8.2144 & 11.5436 \\
\hline 1555 & 0.9475 & 2.8633 & 0.0369 & 3.8478 \\
\hline 1556 & 0.3172 & 0.2553 & 4.2403 & 4.8128 \\
\hline 1557 & 1.1339 & 1.0411 & 5.9328 & 8.1078 \\
\hline 1558 & 0.5017 & 1.0056 & 8.6497 & 10.1569 \\
\hline 1559 & 0.6167 & 0.6111 & 5.9308 & 7.1586 \\
\hline 1560 & 0.9836 & 5.7425 & 6.3792 & 13.1053 \\
\hline 1561 & 0.3281 & 1.7994 & 1.9386 & 4.0661 \\
\hline
\end{tabular}




\begin{tabular}{|l|l|l|l|l|}
\hline $\begin{array}{l}\text { Day } \\
\text { Index }\end{array}$ & $\begin{array}{l}\text { Driving Time } \\
{[\mathrm{hr}]}\end{array}$ & $\begin{array}{l}\text { Idle Time } \\
\text { [hr] }\end{array}$ & $\begin{array}{l}\text { PTO Time } \\
\text { [hr] }\end{array}$ & $\begin{array}{l}\text { Total } \\
\text { [hr] }\end{array}$ \\
\hline 1562 & 0.4922 & 4.3639 & 4.8931 & 9.7492 \\
\hline 1563 & 0.8947 & 1.7858 & 8.5883 & 11.2689 \\
\hline 1564 & 0.3031 & 0.2217 & 0.0122 & 0.5369 \\
\hline 1565 & 0.2072 & 0.5206 & 3.4628 & 4.1906 \\
\hline 1566 & 0.1631 & 0.1492 & 1.6822 & 1.9944 \\
\hline 1567 & 0.4189 & 0.9689 & 2.8689 & 4.2567 \\
\hline 1568 & 0.4661 & 1.6072 & 5.3772 & 7.4506 \\
\hline 1569 & 0.5633 & 1.4361 & 3.8328 & 5.8322 \\
\hline
\end{tabular}

